\title{
A STUDY OF PROGRAMMABLE LOGIC CONTROLLERS (PLC) IN CONTROL SYSTEMS FOR EFFECTIVE LEARNING
}

\author{
by \\ Anup Suresh \\ B.Eng., Ryerson University, 2013 \\ A project \\ presented to Ryerson University \\ in partial fulfillment of the \\ requirements for the degree of \\ Master of Engineering \\ in the Program of \\ Electrical and Computer Engineering
}

Toronto, Ontario, Canada, 2015

(C)Anup Suresh, 2015 


\section{AUTHOR'S DECLARATION FOR ELECTRONIC SUBMISSION OF A PROJECT}

I hereby declare that I am the sole author of this project. This is a true copy of the project, including any required final revisions, as accepted by my examiners.

I authorize Ryerson University to lend this project to other institutions or individuals for the purpose of scholarly research.

I further authorize Ryerson University to reproduce this project by photocopying or by other means, in total or in part, at the request of other institutions or individuals for the purpose of scholarly research.

I understand that my thesis may be made electronically available to the public. 


\begin{abstract}
A Study of Programmable Logic Controllers (PLC) in Control Systems For Effective Learning Master of Engineering, 2015

Anup Suresh

Department of Electrical and Computer Engineering, Ryerson University
\end{abstract}

PLC controllers in today's day are a staple mechanism to control operation of large number of machines and devices in the industry. With their advanced usage, it is increasingly becoming a staple and important part of Engineering. Thus, it is crucial that this knowledge is effectively delivered to students with practical applications.

This paper presents a series of laboratory experiments for students to learn and explore the various industrial applications of PLC's. The control problems in this paper are defined with respect to their applications in different industries such as automotive, steel, oil and electronics. Applications are typical processes that can be observed in these industries such as material conveying, material handling, cutting processes, system control and temperature control. All the problems are solved using Ladder Logic programming on Automation Studio to simulate these processes and provide students with a wholesome learning experience. 


\section{ACKNOWLEDGEMENTS}

Dr. Kaamran Raahemifar, PhD, P.Eng., Ryerson University

I am grateful to my supervisor for the opportunity to pursue this Master's project in a specialized area of interest and wish to express my sincere appreciation and gratitude to him for his guidance throughout the course of this project. His timely feedback and support kept me motivated and gave me confidence to complete this project. In spite of his busy schedule, his time management skills is a quality I will carry forward even after this project.

Vipul Sehrawat, M.A.Sc., Ryerson University

Sincere thanks to Mr. Sehrawat for his guidance and tips throughout the course of this project and my Master's degree.

Shahini Sirikantharajah, M.A.Sc., Ryerson University

I would also like to thank Ms. Sirikantharajah for her valuable inputs and feedback to help me improve my report as well as for her guidance during my degree. 


\section{DEDICATION}

This thesis work is dedicated to my parents and my brother who have been my pillars of strength throughout my life. Without their love, support and encouragement, I would not have made it this far. 


\section{TABLE OF CONTENTS}

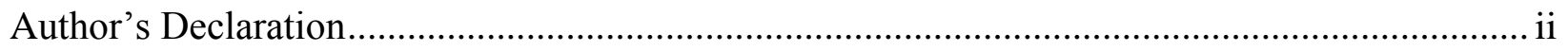

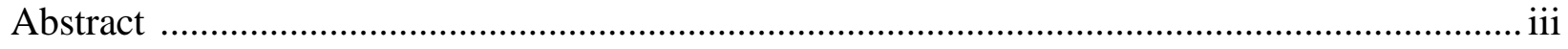

Acknowledgements .................................................................................................. iv

Dedication

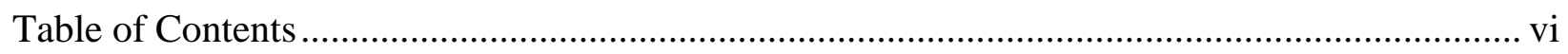

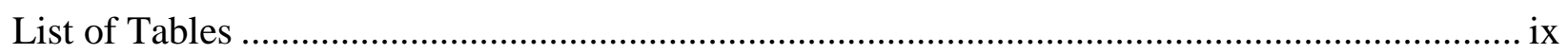

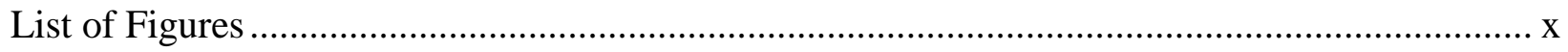

List of Appendices .................................................................................................... xiv

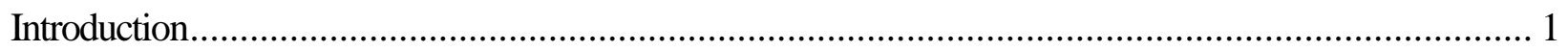

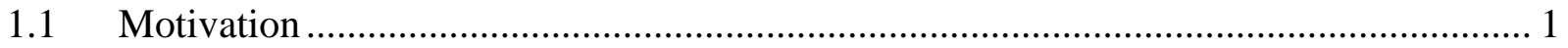

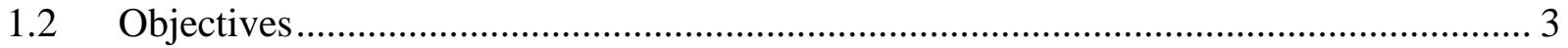

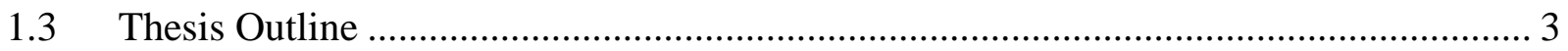

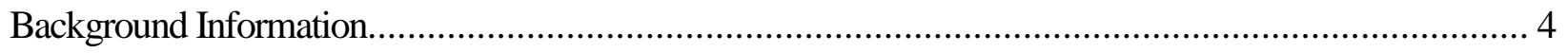

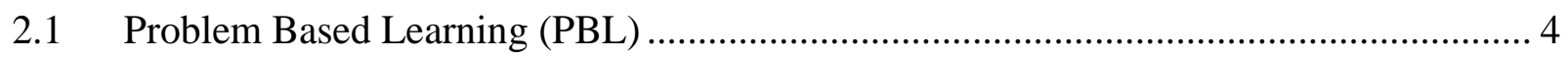

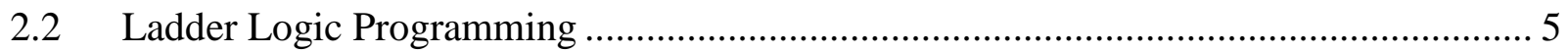

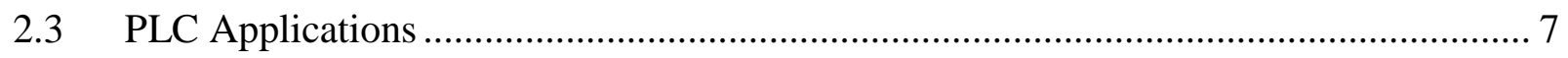

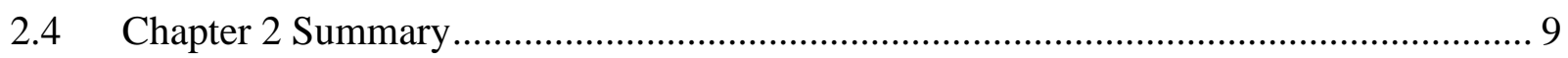

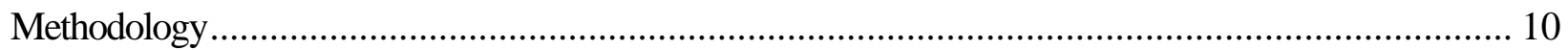

3.1 Initial Phase (Preparation) …………………...................................................... 10

3.2 Experimentation Phase _......................................................................................... 11

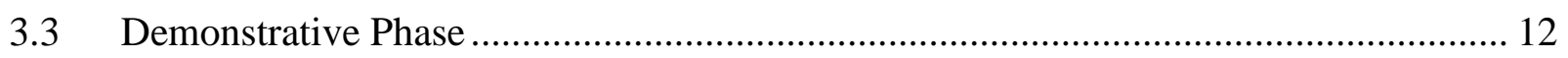

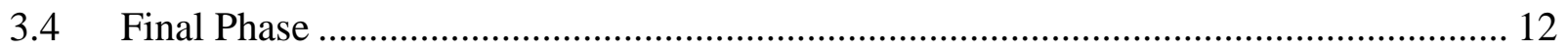

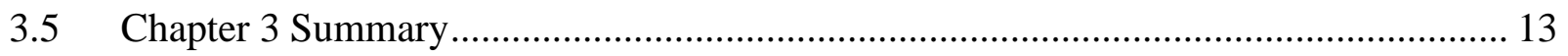




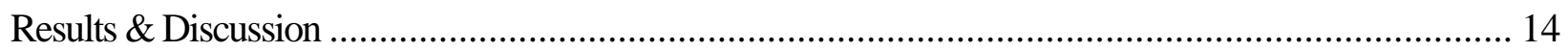

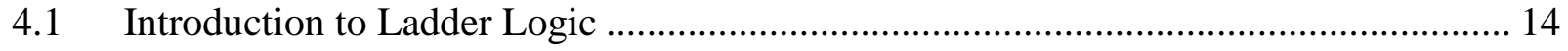

4.2 Applications in the Electronics \& Avionics Industry................................................... 15

4.3 Applications in the Oil \& Chemical Industry ............................................................. 17

4.4 System Control Applications (Automotive \& Aerospace)............................................... 19

4.5 Applications in the Steel \& Manufacturing Industry .................................................... 26

4.6 Applications in the Power \& Gas Industry .................................................................. 28

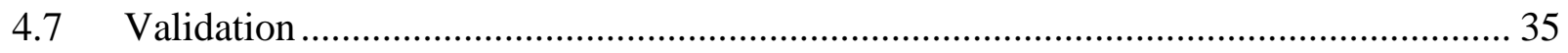

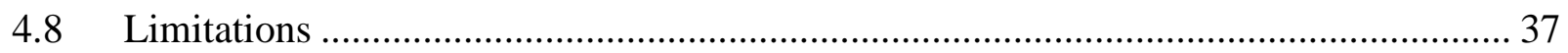

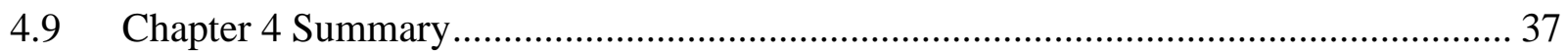

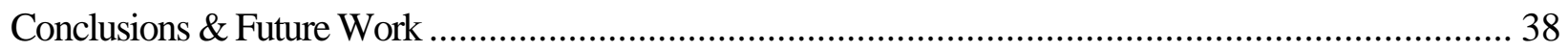

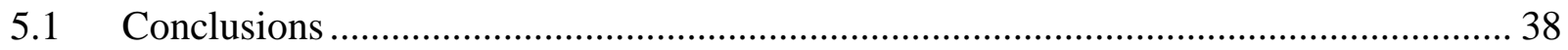

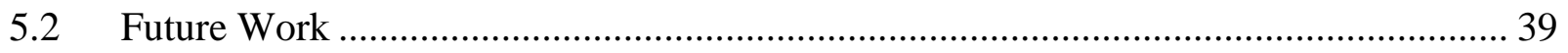

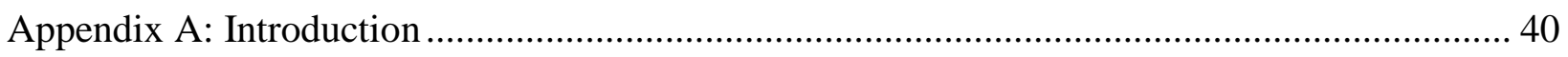

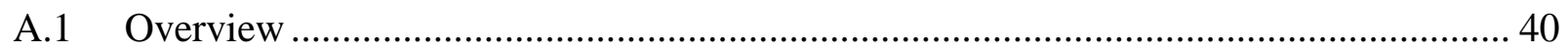

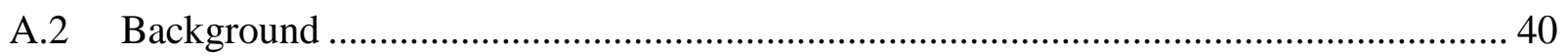

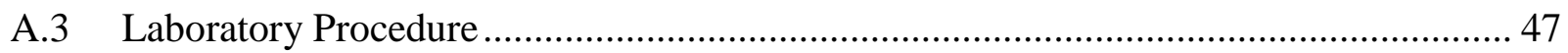

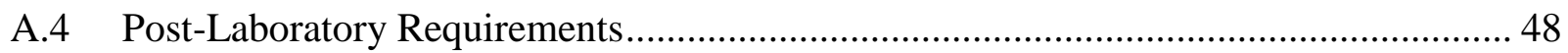

A.5 Laboratory One - Solutions ………………………................................................... 48

Appendix B: Applications in Electronics \& Avionics Industries ……………………………....... 51

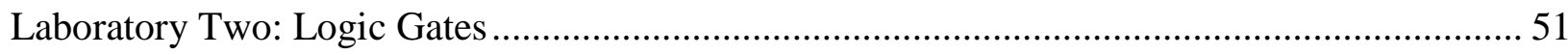

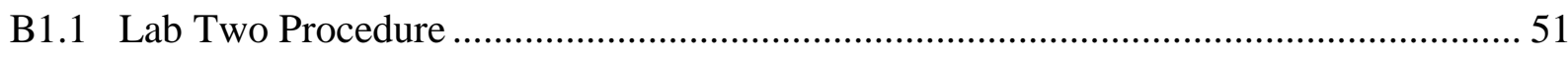

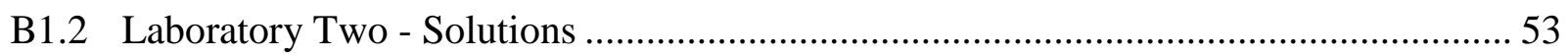

B2.1 Laboratory Three Procedure................................................................................... 57

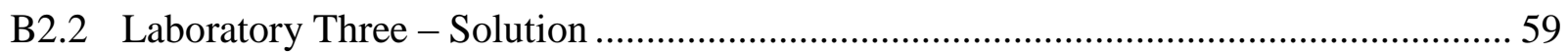

Appendix C: Applications in the Oil \& Chemical Industry ........................................................... 61

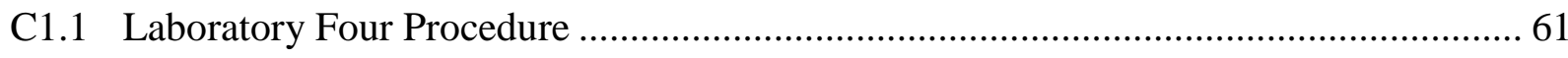




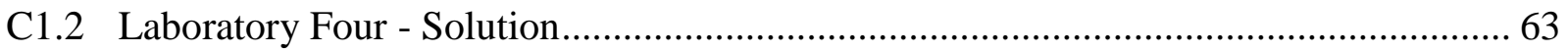

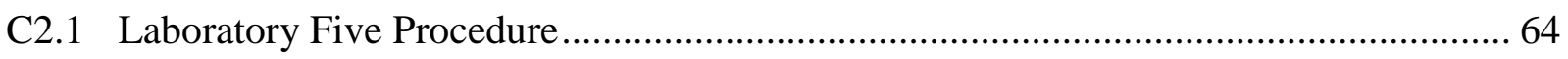

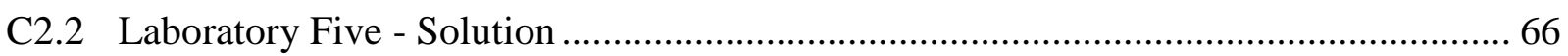

Appendix D: Applications in the Automotive \& Aerospace Industry ........................................... 67

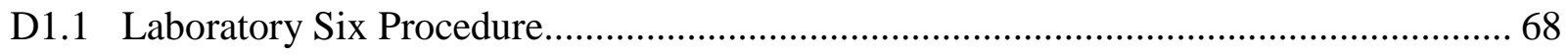

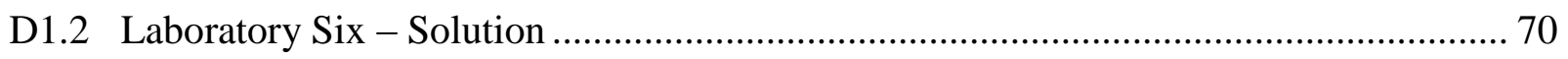

D.2.1 Laboratory Seven Procedure ……………………………………………………..... 71

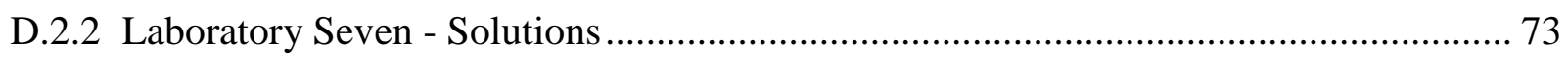

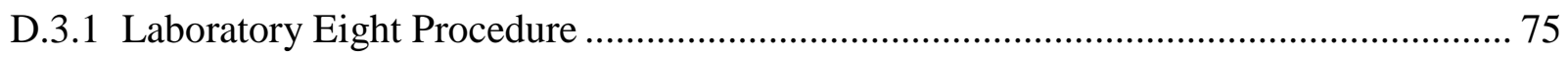

D3.2 Laboratory Eight - Solution ................................................................................... 77

Appendix E: Applications in the Steel \& Manufacturing Industries ............................................ 80

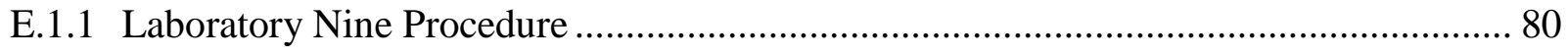

E.1.2 Laboratory Nine - Solution.................................................................................. 82

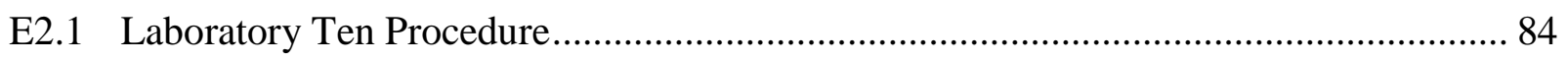

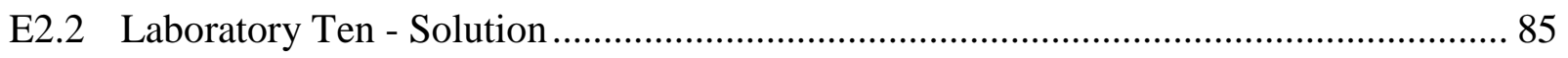

E3.1 Laboratory Eleven Procedure .................................................................................. 86

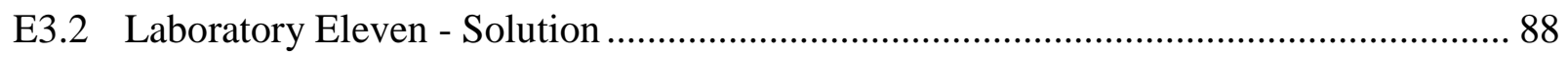

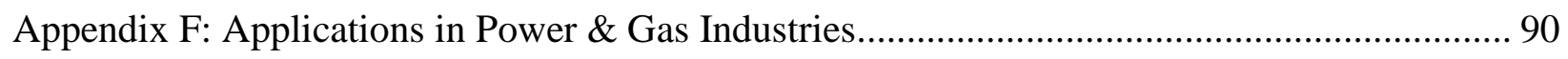

F1.1 Laboratory Twelve Procedure …………………........................................................... 91

F1.2 Laboratory Twelve - Solutions ………………………………………………........ 92

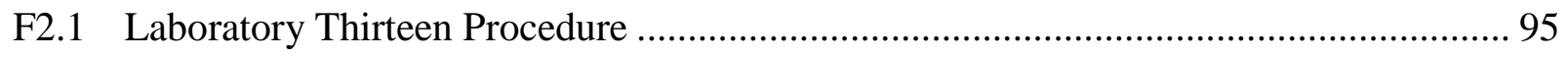

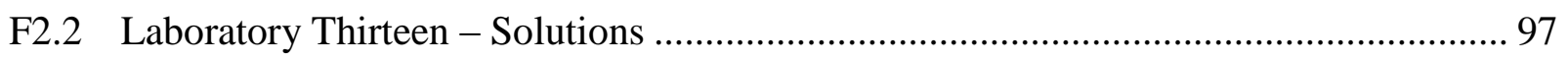

F3.1 Laboratory Fourteen Procedure............................................................................... 101

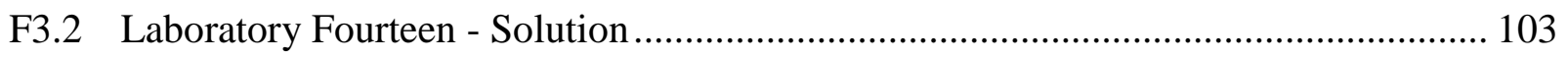




\section{LIST OF TABLES}

Table 3.1: Phases of the Constructivism and Collaborative Principle ............................................ 11

Table 4.1: Address Map for Part 2 of the Logic Gate control problem..................................... 16

Table 4.2: Questionnaire presented to 5 students as a part of the validation study ...................... 36

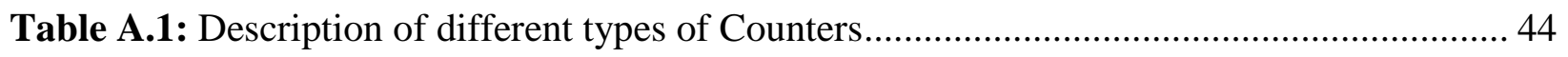

Table A.2: I/O Address Map for Problem................................................................................... 47

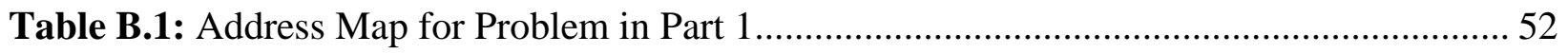

Table B.2: Address Map for Problem in Part 2................................................................ 53

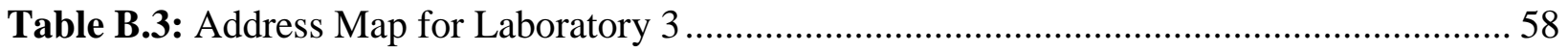

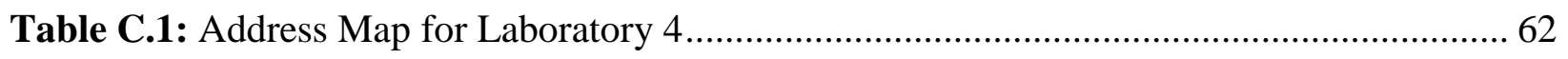

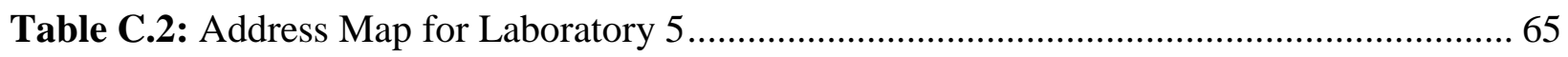

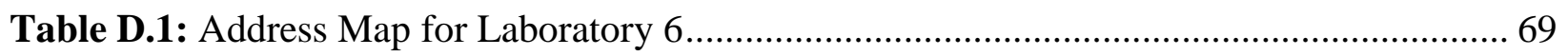

Table D.2: Address Map for Lab 3 Control Problem .............................................................. 72

Table D.3: Address Map for Problem in Part 1 .......................................................................... 75

Table D.4: Address Map for Problem in Part 1 ............................................................................ 76

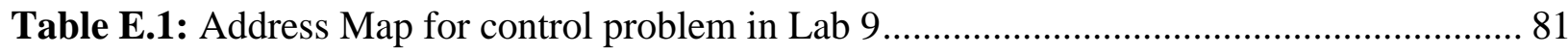

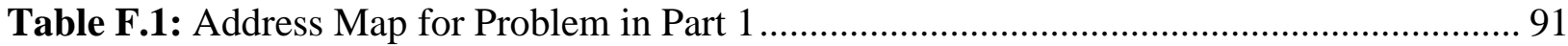

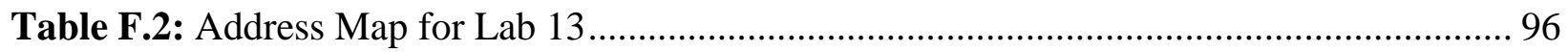

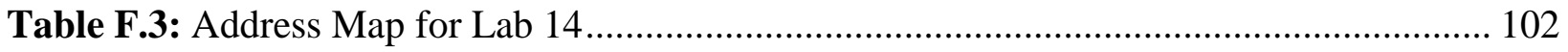




\section{LIST OF FIGURES}

Figure 1.1: Typical PLC system interface between inputs and outputs...................................... 2

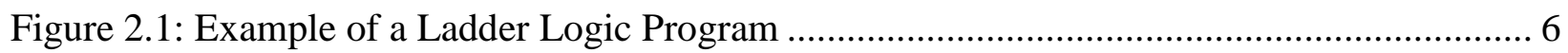

Figure 2.2: Block diagram demonstrating how PLCs are used in the protection of induction

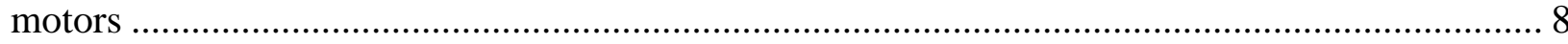

Figure 2.3: Block Diagram of the Water Tank System ………………………………............ 9

Figure 4.1: Ladder logic program for the Logic Gate Control Problem........................................ 16

Figure 4.2: Bottle Filling Operation - Control Problem for Lab 5 …….................................... 18

Figure 4.3: Digital Circuit Wiring Diagram for the 3 Stage A/C System..................................... 20

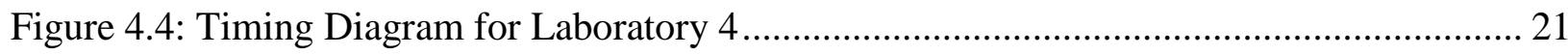

Figure 4.5: Ladder Logic Program for Part 2 of Laboratory 4 ................................................... 22

Figure 4.6: Depiction of the Water Level Monitoring Problem .................................................. 23

Figure 4.7: I/O Wiring Diagram on the PLC Modules ............................................................ 24

Figure 4.8: Ladder Logic Program for the Water Tank Control Problem .................................... 25

Figure 4.9: Automatic Stacking Control problem for Laboratory 9 …………………............... 27

Figure 4.10: Animation of the Double-Acting Pneumatic Cylinder in Automation Studio ......... 29

Figure 4.11: Wiring Connections to the Double Acting Cylinder to begin operation ................... 30

Figure 4.12: A section of the Ladder Logic Program for Lab 5 ................................................... 31

Figure 4.13: Ladder Logic program using the CTD operation ................................................ 32

Figure 4.14: Wiring Diagram for the Motor Connection external to the PLC Module ................. 33

Figure 4.15: Section of the Ladder logic program in Forward Operation .................................... 34

Figure 4.16: Cross-section of the nacelle present in the Wind Turbine........................................ 35

Figure A.1: Basic Ladder Logic Rung Instruction..................................................................... 41

Figure A.2: Types of Timers use in PLC Ladder Logic .............................................................. 42

Figure A.3: Library Explorer on Automation Studio............................................................. 45 
Figure A.4: Component Properties (in order to change Timer Properties).............................. 46

Figure A.5: Variable Manager (to add, delete, modify \& manage variables) ........................... 46

Figure A.6: PLC Input Module connections when NPN sensors are being used (left) and when

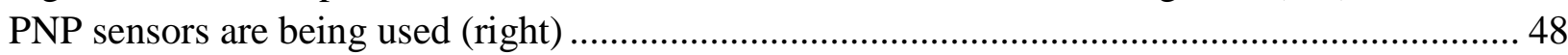

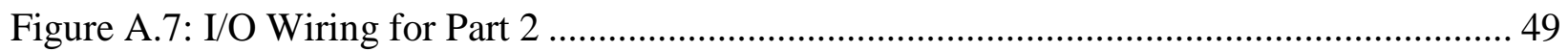

Figure A.8: Ladder Logic Diagram converted from Structure Text ........................................ 49

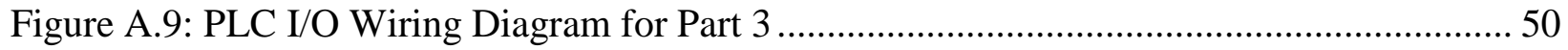

Figure A.10: Ladder Logic Diagram for Part 3 ................................................................. 50

Figure B.1: Digital Logic Circuit for Problem Part 2 …...................................................... 52

Figure B.2: I/O Wiring Diagram indicating Output Y in OFF state when the input switches are CLOSED.

Figure B.3: I/O Wiring Diagram indicating Output $\mathrm{Y}$ in the $\mathrm{ON}$ state when the input switches are

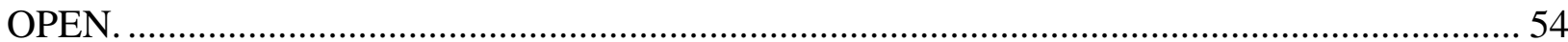

Figure B.4: Ladder Logic Program for Method 1 …............................................................. 54

Figure B.5: Ladder Logic Program for Method 2 using the status bit internal variable............. 54

Figure B.6: Wiring Diagram on PLC Modules for Part 2 the Output Y is in the OFF state ....... 55

Figure B.7: Wiring Diagram on PLC Modules for Part 2 the Output Y is in the ON state ......... 56

Figure B.8: Ladder Logic Program for Laboratory 2 ....................................................... 56

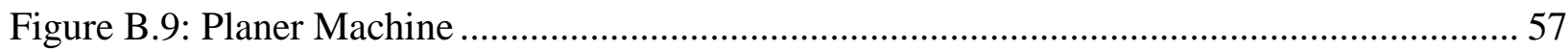

Figure B.10: Digital Logic Wiring Diagram for the Planer Machine Control System ............... 58

Figure B.11: PLC I/O Wiring Diagram for the Planer Machine Control System ...................... 59

Figure B.12: Ladder Logic Program for the Planer Machine Control System .......................... 60

Figure C.1: Lab 4 - Batch Mixing System Diagram ...................................................... 61

Figure C.2: I/O Wiring Diagram for the Batch Mixing system .......................................... 63

Figure C.3: Ladder Logic program for the Batch Mixing system ............................................ 63

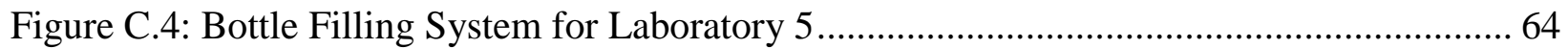

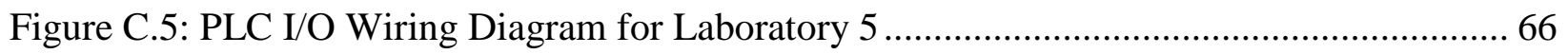

Figure C.6: Part 1 of the Ladder Logic program for Laboratory 5 .......................................... 66

Figure C.6: Part 1 of the Ladder Logic program for Laboratory 5 ........................................... 66

Figure C.7: Part 2 of the Ladder Logic program for Laboratory 5 .......................................... 67 


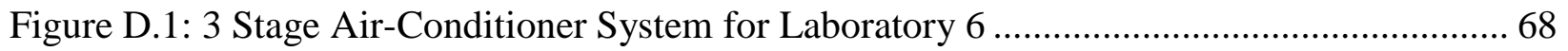

Figure D.2: PLC I/O Wiring Diagram for Laboratory 6................................................... 70

Figure D.3: Ladder Logic program for Laboratory 6 ..................................................... 70

Figure D.4: Illustration of the Water Tank Monitoring System ......................................... 71

Figure D.5: I/O Wiring Diagram on the PLC Modules for Lab 3 ........................................... 73

Figure D.6: I/O Wiring Diagram on the PLC Modules for Lab 3 when the water level is HIGH 73

Figure D.7: I/O Wiring Diagram on the PLC Modules for Lab 3 when the water level is LOW 74

Figure D.8: Ladder Logic Program for Lab 3 ....................................................................... 74

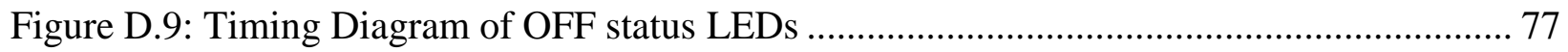

Figure D.10: I/O Wiring Diagram demonstrating the Fan in OFF state................................. 77

Figure D.11 I/O Wiring Diagram demonstrating the Fan in ON state.................................... 77

Figure D.12: Ladder Logic Diagram for Laboratory 4 - Part 1 ........................................... 78

Figure D.13: I/O Wiring Diagram demonstrating the Motor and Fan in operation..................... 78

Figure D.14: I/O Wiring Diagram displaying the Motor OFF................................................ 78

Figure D.15: Ladder Logic Diagram for Laboratory 4 - Part 2 ........................................... 79

Figure E.1: Automatic Conveyor Stacking System .......................................................... 81

Figure E.2: PLC I/O Wiring Diagram for Lab 9 ............................................................. 82

Figure E.3: Ladder Logic program for the Automatic Conveyor Stacking System ................... 83

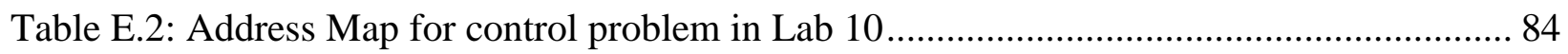

Figure E.4: PLC I/O Wiring Diagram for the Product sorting system ................................... 85

Figure E.5: Part 1 of the Ladder Logic program for the Product sorting system ...................... 85

Figure E.6: Part 2 of the Ladder Logic program for the Product sorting system ...................... 86

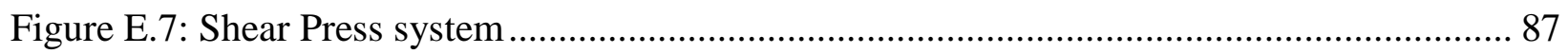

Table E.3: Address Map for control problem in Lab 11 ................................................... 88

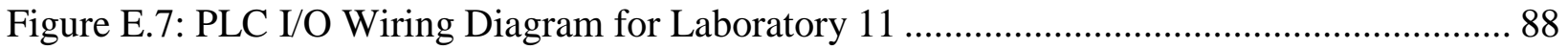

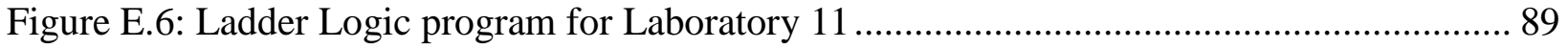

Figure F.1: Visualization of Control problem for Laboratory 5 ....................................... 90

Figure F.2: Wiring Connections to the Double Acting Cylinder to begin operation................... 92

Figure F.3: I/O Wiring Diagram to the PLC Module for Lab 5 ............................................ 93

Figure F.4: Ladder Logic Diagram Part $1\left(1^{\text {st }}\right.$ Failure Event Clocked at $\mathrm{t}=$ 00:01:42)............. 93 
Figure F.6: Visualization of the Control Problem

Figure F.7: Complete Solution for Laboratory 6 including Wiring Diagrams and Ladder Logic 97

Figure F.8: Motor operating in FORWARD operation ...................................................... 98

Figure F.9: Motor operating in REVERSE operation..................................................... 98

Figure F.10: I/O Wiring Diagram to the PLC module for Laboratory 6 ................................. 99

Figure F.11: Ladder Logic Diagram for Motor Forward Operation ....................................... 99

Figure F.12: Ladder Logic Diagram for Motor Reverse Operation....................................... 100

Figure F.13: Retentive Timer Logic Design \& Reset Operation ............................................. 100

Figure F.14: Nacelle cross-section of the Wind Turbine system ........................................ 101

Figure F.15: PLC I/O Wiring Diagram for Lab 14 (currently in Strong Wind Status) ............. 103

Figure F.16: Part 1 of the Ladder Logic Program for Lab 14 .............................................. 103

Figure F.17: Part 2 of the Ladder Logic Program for Lab 14 ............................................... 104

Figure F.18: Sub-Routine Ladder Logic Program for Lab 14 .............................................. 104 


\section{LIST OF APPENDICES}

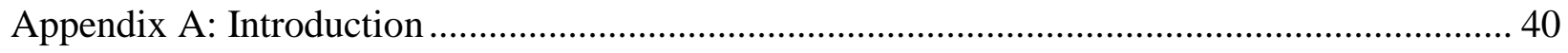

Appendix B: Applications in Electronics \& Avionics Industries ................................................... 51

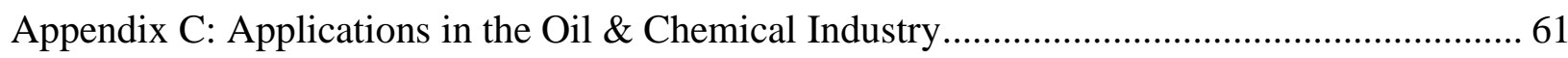

Appendix D: Applications in the Automotive \& Aerospace Industry ........................................... 67

Appendix E: Applications in the Steel \& Manufacturing Industries ............................................. 80

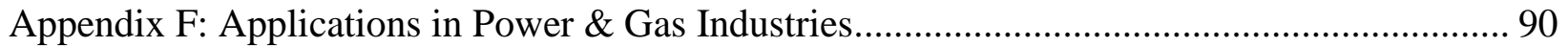




\section{Chapter 1}

\section{Introduction}

\subsection{Motivation}

LC's or programmable logic controllers are one of the most widely used industrial control 1 systems used today to enhance the functionality of a production line or machine functions. Their flexibility in controlling a wide range of inputs and outputs and changing their operation based on requirements have made them a favourite control system in recent times.

The typical PLC system is like any other computer system that consists of various hardware components such as the CPU, programming devices as well as external hardware components Input/Outputs (I/O) and power supply. The CPU system is microprocessor based that allows all arithmetic operations as well as logic operations, blocks memory moves, handles the computer interface etc. Typical systems have 4 buses: a data bus to share information between different elements, address bus for the respective addresses, control bus for internal actions that need to be monitored and lastly a system bus which allows the communication between the various ports and Input/output unit [1]. Inputs are usually sensors or switches that control outputs such as motors and electric pumps etc.

Thus, similar to other computerized devices, the PLC unit has a memory storage as well as power supply thus making it a very small integrated unit that can be used in various industries for a wide variety of applications. 


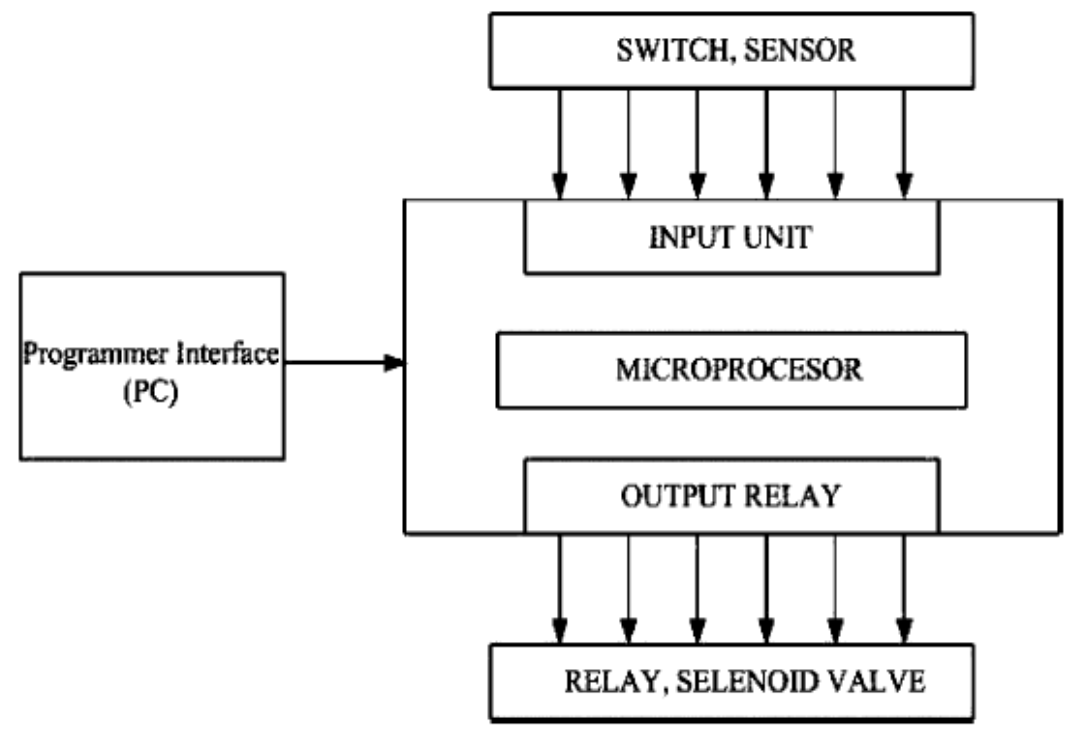

Figure 1.1: Typical PLC system interface between inputs and outputs [1]

The criteria for selection of an appropriate PLC varies from system requirements and application requirements to physical environments. For any application, it is extremely crucial to determine that the PLC can handle the application in the appropriate way. After the goals of the application are determined, the inputs and outputs need to be identified that are required by the system. Once these factors are considered, the electrical requirements come into play and the input power, device voltage and currents need to be calculated. This is followed by the communication protocol that will be used by the PLC which can be of various types. In Automation studies and control systems, theoretical information along with constraints and specifications play a key role in solving different types of control problems. It is critical that none of these factors are ignored and the most efficient process is developed.

In today's day and age, it is important that university students are well versed with industrial practices and introduced to practical applications to prepare them for their careers. Currently, education is limited to theory and background of programming tools but students are not tested on critical thinking and unable to identify real control applications where PLCs are used. 


\subsection{Objectives}

The primary objective of this project thesis was to develop a series of laboratory experiments related to Programmable Logic Controllers for students with no prior experience in industrial automation and control systems. The secondary objective of this project was to provide a wholesome learning experience for students by developing practical applications that would prepare students to actively use this knowledge in the industry or internship.

It is hypothesized that 14 laboratory exercises would provide students with a sufficient grasp of Automation principles and ladder logic programming in the different industries.

\subsection{Thesis Outline}

The thesis outline is as follows:

- Chapter 2: This chapter details the background information that is related to PLCs and provides a literature review to critique and analyze currently existing research and experimental methodologies related to PLC experimental designs, training, troubleshooting and fault detection.

- Chapter 3: The methodology used to develop the laboratory experiments has been discussed in this chapter which consists of 4 different phases. Chapter outlines these phases along with the activities that are encompassed in each of them to provide a wholesome learning experience for students.

- Chapter 4: All the laboratory exercises have been briefly described and ladder logic examples have been presented here. The learning objectives of each exercises and the results and outcomes of each laboratory procedure is discussed. The chapter ends by discussing a validation study that was conducted and the results and limitations of the study.

- Chapter 5: In the final chapter, this entire project thesis has been summarized along with the objectives and results. Furthermore, the future work and potential areas of improvements has been discussed. 


\section{Chapter 2}

\section{Background Information}

\subsection{Problem Based Learning (PBL)}

$\mathrm{D}$ esign of experiments for practical classes often employs the PBL method. The PBL method suggests that exercises be offered to students along with theoretical knowledge to test the understanding of the material as well as promote an experiential learning process. Numerous educational institutions have employed this method and developed exercises in the context of PLC programming. Examples of these are the traffic light simulation, batch mixing and continuous bottle filling system which is commonly found as a practical exercise in most PLC programming laboratories.

One such implementation of the PBL method has been employed by universities that use a PLC and SCADA (Supervisory Control and Data Acquisition) based laboratory exercises to enable students to learn to control and monitor industrial processes [2]. The software required for the execution of these laboratories is not only expensive, but also require students to quickly grasp the concepts of PLC when they have no prior experience in automation. SCADA systems incorporate numerous subsystems such as terminal units (RTUs), HMI (Human Machine Interface) and PLCs that need to be controlled together to perform a task or operation [2]. These systems are typically at a remote location that allows user to monitor that status of an operation or equipment from a distant location. Laboratories incorporating the SCADA system requires students to actively develop a Graphical User Interface (GUI) and establish a communication to the PLC system. Successful implementations of GUI and communication protocols are determined by visualizing the outputs connected to the PLC modules. It must also be noted that most of these laboratories were designed for students in their final year of studies as they are well acquainted with electrical theory and terminology. 
In yet another PBL application related to PLCs, mechatronics laboratories were designed to use the RS Logix 500 PLC and a GUI to control light stacks and conveyor systems for materials handling [3]. The designed experiments employ a practical hands-on learning experience for students to control materials on conveyor belts, optically detect the placement of materials and use sensors and actuators to control the objects on the conveyor belts. The experiments take the advantage of sponsored automation equipment and actively engage students in programming and controlling of electro-mechanical systems to provide an experiential learning process for future career opportunities.

Contrast to the PBL method of learning, educational institutes have also introduced a remote learning experience with distance learning becoming a practical and common education norm in today's learning experience. These experiments provide students with GUIs and HMI interfaces to actively control and receive data from a remote laboratory equipment but fail to provide a practical application of the control systems. Students are typically required to obtain electrical characteristics such as voltages, current and acceleration-deceleration profiles [4]. Movements of sensors and actuators are observed through a webcam placed at the remote e-laboratory. The experimental setup is typically expensive and poses safety questions as there is no one to monitor the equipment in real-time at the remote location. Although, most PLC remote stations do incorporate safety measures and limit the control of the equipment from a remote distance, these systems can be designed on a design software where these industrial processes and equipment can be replicated.

\subsection{Ladder Logic Programming}

One of the widely used methods of controlling PLCs is using Ladder logic programming. The ladder logic diagram is a representation of the steps of programming that employs relay coils and contacts to control external outputs and actuators. These outputs can be such as indicator lights, alarms as well as solenoids, motors and pneumatic cylinder amongst others. The ladder program is all PLCs follows a template that consists of 2 bus bars that are connected to one another using rung lines. 


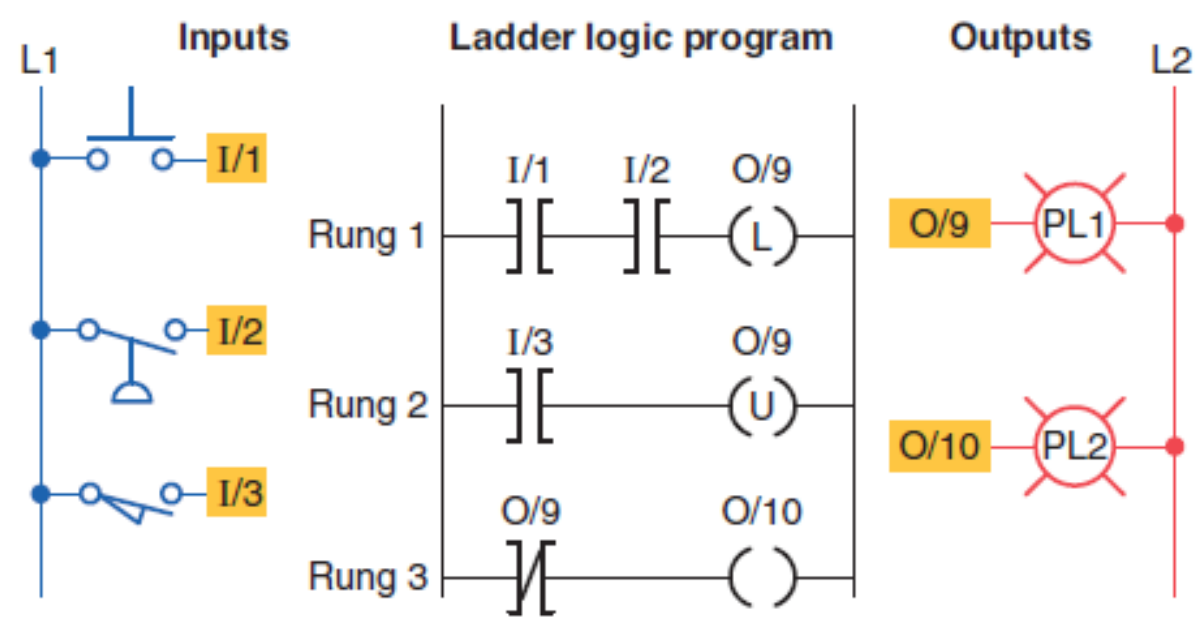

Figure 2.1: Example of a Ladder Logic Program [5]. The 2 bus bars on either side are connected by Rungs 1, 2 and 3. L1 and L2 are the power sources to the input and output instructions. Notice how each contact coil on the rungs also has a respective address mapped to the input and output instructions which is indicated above the instruction

Bus bars, also known as power rails supply the power from the PLC to the instructions it contains. The instructions towards to the left bus bar are input instructions which typically consist of Normally-Open (NO), Normally-Closed (NC) contacts coils and status bits that are used to control output instructions. These output instructions are indicated towards the right bus bar which can include external output instructions, mathematical operations, conversions, timers, counters and other PLC instructions. The rung lines that connect the 2 bus bars are on which the instructions are written which can be written in a single rung or used in a logic connection such as "OR" or "AND" along with a second rung line [5]. Thus, they are also sometimes referred to as branching instructions. When an input logic instruction is true, power flows from the left bus bar to the right bus bar thereby energizing the output instructions and perform an operation or task. Manipulation of these instructions and control of input/output instructions allows users to solve control problems in an efficient manner.

Every PLC program instruction, whether simple or complex requires designers and programmers to follow few basic steps such as:

1. PLC architecture and PLC I/Os need to be clearly defined [5]. 
2. Concepts of ladder logic programming must be well understood and it is critical that the symbols for all contact coils and output instructions are well understood.

3. Sequential steps of control problems must be clearly defined to identify which instructions must be written first and on which rung the operation must be performed.

4. A large number of PLCs are operated in automation industries to control pneumatics and motorized machines. Thus, concepts of motor theory and pneumatic cylinders must be well understood.

5. Circuit diagrams for PLC inputs and outputs need to be drawn to identify which input instruction is responsible for the different output operations.

\subsection{PLC Applications}

PLC's have grown to be one of the widely used devices in all industries to simplify and automate processes. Using PLCs significantly reduces the work for electrical modifications and provides flexibility to change the operation of a machine as desired.

A major application of PLCs is to develop a new protection system for induction motors (IM) [1]. A lot of the current methods for fault detection do not hold the ability to detect the mechanical or electrical failures (such as stator insulation failures) in time and thereby reduce the life of an operation system. Monitoring electrical characteristics such as current, voltage, speed and temperature can be done using a PLC. A measurement card consisting of voltage and current transformers are set up to measure the phase currents and voltages of the IM. Furthermore, a temperature sensor and incremental encoder are also introduced to measure the temperature and the rotor speed of the IM respectively.

This measurement system is connected to an analog module that converts the analog values obtained from the motor to a digital value that can be read by the PLC and interpreted on a computer. Therefore, any irregular deviations in these values result in the PLC stopping the motor and giving an alarm message on the computer.

Another such application is that of a PLC based control system employed in a geothermal plant. In the plant, $\mathrm{CO} 2$ is the primary working fluid for conversion of geothermal water to electrical 
energy [6]. Hence, the PLC control system must maintain a constant CO2 pressure and temperature during all the phases of its thermodynamic cycle [6].

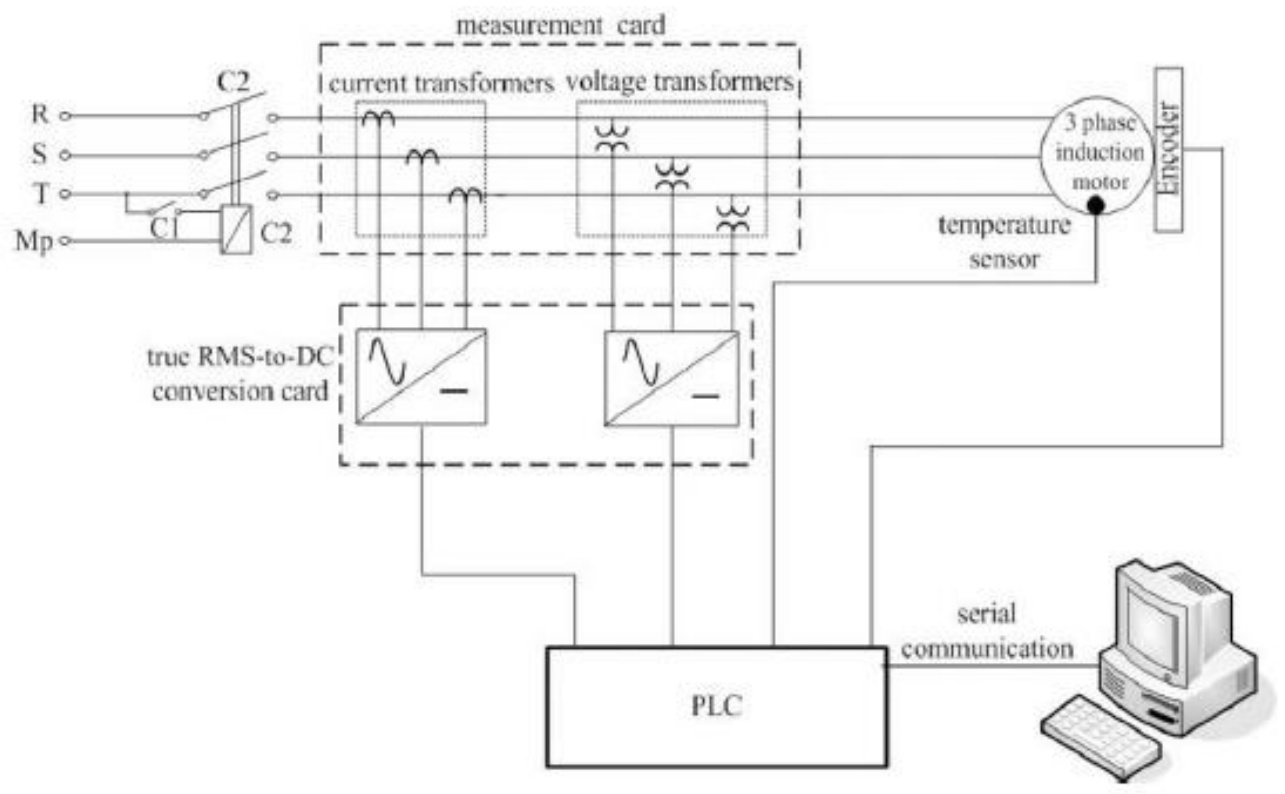

Figure 2.2: Block diagram demonstrating how PLCs are used in the protection of induction motors [1]

Thus, a constant temperature needs to be maintained after vaporizing the $\mathrm{CO}_{2}$ which is done using redundant temperature transducers and monitored on a remote computer [6]. Any deviations in the values of one of the transducers causes the PLC to switch the system to use the other transducer and continue normal operation, a method commonly known as Interlocking where two outputs are not active at the same time.

PLCs are also widely used in water pump control systems for production plants. The production plants often contain harsh environments wherein constant modifications to wiring and cabling can cause severe damage and stop the operation of the plant [7]. In these cases, PLCs are used to control the water pumps to turn on and off. Pressure transmitters are used along with limit switches to obtain the level of the water in the tank system [7]. In the event that the level of the water in the tank system is outside the desired range of operation, the values from the limit switches can be detected with the PLC on the computer system to operate the water pump. 
This process is depicted in Figure 2.3 below. Smaller applications of PLC systems are such as those that are employed in elevator systems wherein when a button is pressed, the PLC switches the input to the respective output instruction to move the elevator to the respective floors. PLCs are also used to control traffic lights and alternate between the lights at an intersection.

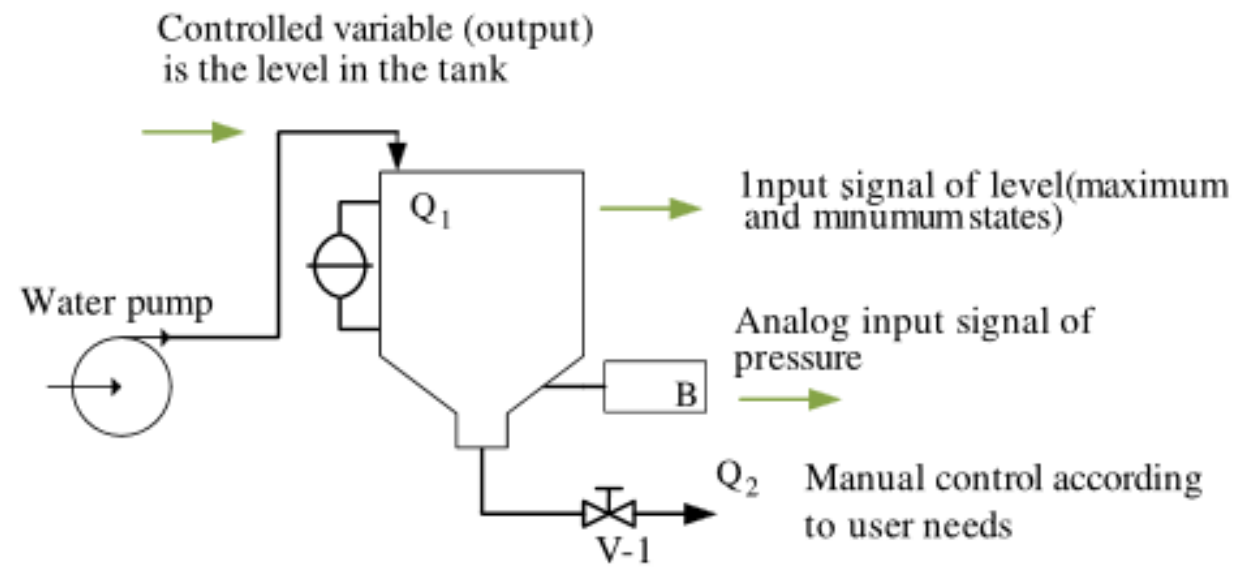

Figure 2.3: Block Diagram of the Water Tank System [7]. One of the major applications where PLCs are commonly used to determine the level of the water in the tank from a remote station

Simple control systems are also designed where PLCs are used to detect faults following which an appropriate flag is set in the PLC program which performs the necessary output actions. Thus, PLCs have a wide range of applications with their uses found in larger manufacturing plants as well as smaller applications and fault-monitoring operations from where diagnostic reasoning and knowledge can be deduced.

\subsection{Chapter 2 Summary}

This chapter presents a literature review on the Problem Based Learning (PBL) methodology which is employed by educational institutes to prepare practical laboratory exercises. The different types of exercises that can be created are discussed along with a background on ladder logic programming. Finally, the applications of Programmable Logic controllers is provided to demonstrate why they are one of the most widely used controllers today. 


\section{Chapter 3}

\section{Methodology}

$\mathrm{I}$

n order to design laboratory exercises and deliver an experiential learning process to students,

the principles of collaborative and constructivism were followed [9, 10]. This methodological procedure typically consists of 4 phases - the preparation phase, experimentation phase, demo phase and the final phase. These phases include activities and require a time duration for execution which has been allocated in Table 3.1.

\subsection{Initial Phase (Preparation)}

The initial phase in this methodology is where theoretical knowledge is delivered to students in the form of lecture classes or pre-laboratory lecture [9]. The initial phase also involves self-study by students wherein students are required to review the laboratory procedure prior to performing the exercises. In order to perform the exercises here, students would be required to explore the topic outside of the lab and gain the knowledge required to perform the exercises. Typically, this phase would also involve the creation of teams wherein 2 students are paired with one another to perform the laboratory exercises. Other activities in this phase include answering pre-laboratory questions or developing strategies to solve the problems presented.

Since all the control problems in these laboratories are bound by constraints and specifications, a process flow diagram could be developed to visualize the control problem. Students can then compare there process flow charts to that of other groups to identify the different approaches of solving the control problem. This process not only encourages team work to flourish but also allows students to be involved in a peer-review process. This preparation phase will also involve students and instructor involved in discussions prior to performing the exercises to ensure students are well versed with the concepts and different specifications and constraints in the control problems presented here. 


\subsection{Experimentation Phase}

The experimentation phase involves the process of performing the laboratory procedures. The software tool used to perform the laboratory procedures here is Automation Studio. The software, Automation Studio is an electrical design and simulation software that was developed by Famic Technologies Inc. in the mid-90s [11]. The tool is widely used for training and troubleshooting electrical control systems as well as simulating industrial automation processes to safely validate designs and programs prior to practical applications [11].

In this phase, students will begin be acquainting themselves with the software starting with the first introductory laboratory and progressing towards more complex control problems. All the problems presented here require solving them using ladder logic programming as well as provide wiring diagrams to control external inputs such as switches, pushbuttons and external outputs such as LEDs, indicator lights, motors and pneumatic cylinders. The problems are solved by wiring the I/Os on the respective PLC modules followed by writing the ladder logic program. As students progress towards more complex problems, they will also be required to wire the external actuators such as motors and pneumatic cylinders which will provide them with an experiential learning process.

Table 3.1: Phases of the Constructivism and Collaborative Principle along with the different activities and during of time required to achieve completion using the technical resources

\begin{tabular}{|c|l|c|c|}
\hline Phase & \multicolumn{1}{|c|}{ Activity } & Duration (h) & Technical Resources \\
\hline Preparation & $\begin{array}{l}\text { Lecture classes, pre-laboratory } \\
\text { exercises, team building, self- } \\
\text { study }\end{array}$ & 4 & Multimedia \\
\hline Experimentation & $\begin{array}{l}\text { Laboratory Exercises, Testing, } \\
\text { Simulation }\end{array}$ & 3 & Automation Studio \\
\hline Demonstrative & $\begin{array}{l}\text { Lab presentation, Design } \\
\text { Validation }\end{array}$ & 0.5 & Automation Studio \\
\hline Final & $\begin{array}{l}\text { Post-Laboratory Analysis, } \\
\text { Discussion, Feedback }\end{array}$ & 3 & Multimedia \\
\hline
\end{tabular}




\subsection{Demonstrative Phase}

In the demonstrative phase or the presentation phase, students will be evaluated by their respective instructors on the efficiency and accuracy of their solution to the control

problems $[9,10]$. Students will demonstrate different sections of the laboratory and test different conditions to ensure the solution to the control problem is accurate. Students will be required to verify that all the constraints and specifications have been met as proposed in the laboratory procedures. Moreover, the address map must be followed accurately and the wiring diagrams must be accurate. Different conditions will be checked by the instructor before students can be graded on the completeness of the laboratory procedure.

Modelling and simulation in learning the concepts of ladder logic programming and attempting to solve automation control problems is hugely beneficial as students with no prior experience with PLCs can gradually progress from basic problems to solving more complex problems. This also facilitates the need for communication between group partners and building soft skills such as team work and critical thinking. Finally, this allows students to validate designs and perform each laboratory task by trial and error and visualize the control process. Instructors can also provide support or hints to the students when they are to hit upon roadblocks which ensures an engaging learning process.

\subsection{Final Phase}

The last phase of the principles of collaborative and constructivism involve the analysis of control problems and solutions developed [10]. This requires student to explore the topic outside of the knowledge gained through laboratory procedure and provide a report to the instructor explaining their reasoning behind the design solutions developed. Students are provided a feedback on how to efficiently improve their solutions where necessary and graded on the entire laboratory. This allows students to reflect upon their design choices and gain a better understanding of PLCs and ladder logic programming. In the final phase, a questionnaire can also be provided to students for instructors to receive a feedback on how to improve the exercises and provide an even more engaging and practical learning experience. 


\subsection{Chapter 3 Summary}

This chapter summarizes the principles of constructivism and collaborative learning that is considered good practice in undergraduate education that must be inculcated in all forms of education to the extent possible. The methodology encourages communication and active learning while simultaneously allowing students to develop soft skills in team building and critical thinking and also providing a wholesome learning experience. In the process, prompt feedback from instructors and students helps promote a diverse learning environment to deliver complex theoretical concepts in an engaging manner. This chapter presents the different activities under each phase of the principles along with the time that would typically be required to complete the activities in the phase. 


\section{Chapter 4}

\section{Results \& Discussion}

$\mathrm{T}$

he following section briefly describes the 14 laboratory experiments designed along with their applications and relevance in the industry to which they belong. The experiments designed increase gradually in difficulty from introductory exercises to more complex industrial control problems. All the learning objectives, control problems, results and learning outcomes are described here. Students learn to use detailed specifications and constraints provided to them and formulate a solution using Ladder Logic programming performed on Automation Studio. Each laboratory is designed to cater to students with all levels of experience in PLC programming. Lab experiments are designed to demonstrate the practical applications of PLCs in the industry which would help students be ready to program PLCs in future for any control problems they might be faced with. Apart from PLC programming, students also develop soft skills such as team building and critical thinking by breaking down big problems into individual smaller components.

\subsection{Introduction to Ladder Logic}

This introductory laboratory 1 is designed for students to become accustomed to the Automation Studio software. Automation Studio is an electrical design \& simulation software used for learning and troubleshooting of power, pneumatics and PLC systems [11]. The laboratory provides an overview of the software as well some key tools that are required to navigate through the software and perform the experiments. This introduction laboratory teaches students to create a new project and begin their first attempt at programming PLCs. Students begin from the basics of PLC ladder logic programming by learning about different types of coils and contacts such as Examine If Open (XIO) and Examine If Closed (XIC) contacts as well as 
Output energizing coils. A PLC ladder logic rung diagram is set up along with the input contacts and outputs. Students also learn to wire PLC terminal outputs and inputs as well as learn about the address mapping procedure to program PLCs. Furthermore, all the operations and instructions that will be required to perform the control problems in Laboratory experiments 2 to 6 are described here. Students will use this introduction laboratory as a reference to use necessary instructions and solve the control problems that are presented to them.

\subsection{Applications in the Electronics \& Avionics Industry}

In a lot of industrial applications today, PLCs are widely used to solve and simplify complex problem with constraints. This means that programming must often be performed using logic operators like AND, OR and NOT. The use of these operators with one another helps in solving critical problems while simultaneously satisfying constraints. This is why an understanding of their functionality and operation in combination with one another can be useful to solve various control problems in the field of Electronics \& Avionics. PLCs are widely used for the manufacture of Printed Circuit Boards (PCB) and other products for material handling of electronic parts, inventory control as well as in product conveying systems.

The application in the industry is demonstrated using 2 control labs wherein students use ladder logic to perform digital logic operations as well as learn how to perform a planar machine control - a system widely used in electronics manufacturing operations.

In Part 1 of laboratory 2, a scenario is presented wherein the task is to control an output relay using 2 Normally-Open (NO) switches. This part of the lab requires students to solve the problem in 2 different ways, one of which involved creating a new variable using the variable manager on Automation Studio. As a learning outcome, students learn to wire the input \& output terminals of a PLC as well as create a rung. The goal behind creating a new variable is to demonstrate the use of an internal memory bit in the PLC which holds the status of the output and can be used at a later stage in the rung diagram.

In Part 2 of lab 2, a digital logic circuit is presented to students. The experiment demonstrates a practical control problem where the logic circuit must first be converted to its Boolean expression form and simplified to its canonical form. The 5 inputs are NO switches as the 
external inputs to control a single output LED. The output LED only turns on when the output to the circuit is of the logic ' 1 '.

Table 4.1: Address Map for Part 2 of the Logic Gate control problem. A similar template of the I/O map can be found for all the control problems that follow.

\begin{tabular}{|c|c|c|}
\hline Address & Type & $\begin{array}{c}\text { Description/Functional } \\
\text { Requirement }\end{array}$ \\
\hline I:0/2 & External Input & Switch \#1 \\
\hline I:0/3 & External Input & Switch \#2 \\
\hline I:0/4 & External Input & Switch \#3 \\
\hline I:0/5 & External Input & Switch \#4 \\
\hline I:0/6 & External Input & Switch \#5 \\
\hline B3:0/0 & Internal & Internal Relay 1 \\
\hline B3:0/1 & Internal & Internal Relay 2 \\
\hline O:0/1 & $\begin{array}{c}\text { External } \\
\text { Output }\end{array}$ & $\begin{array}{c}\text { Light which turns ON when the } \\
\text { output to the Logic circuit is '1' }\end{array}$ \\
\hline
\end{tabular}

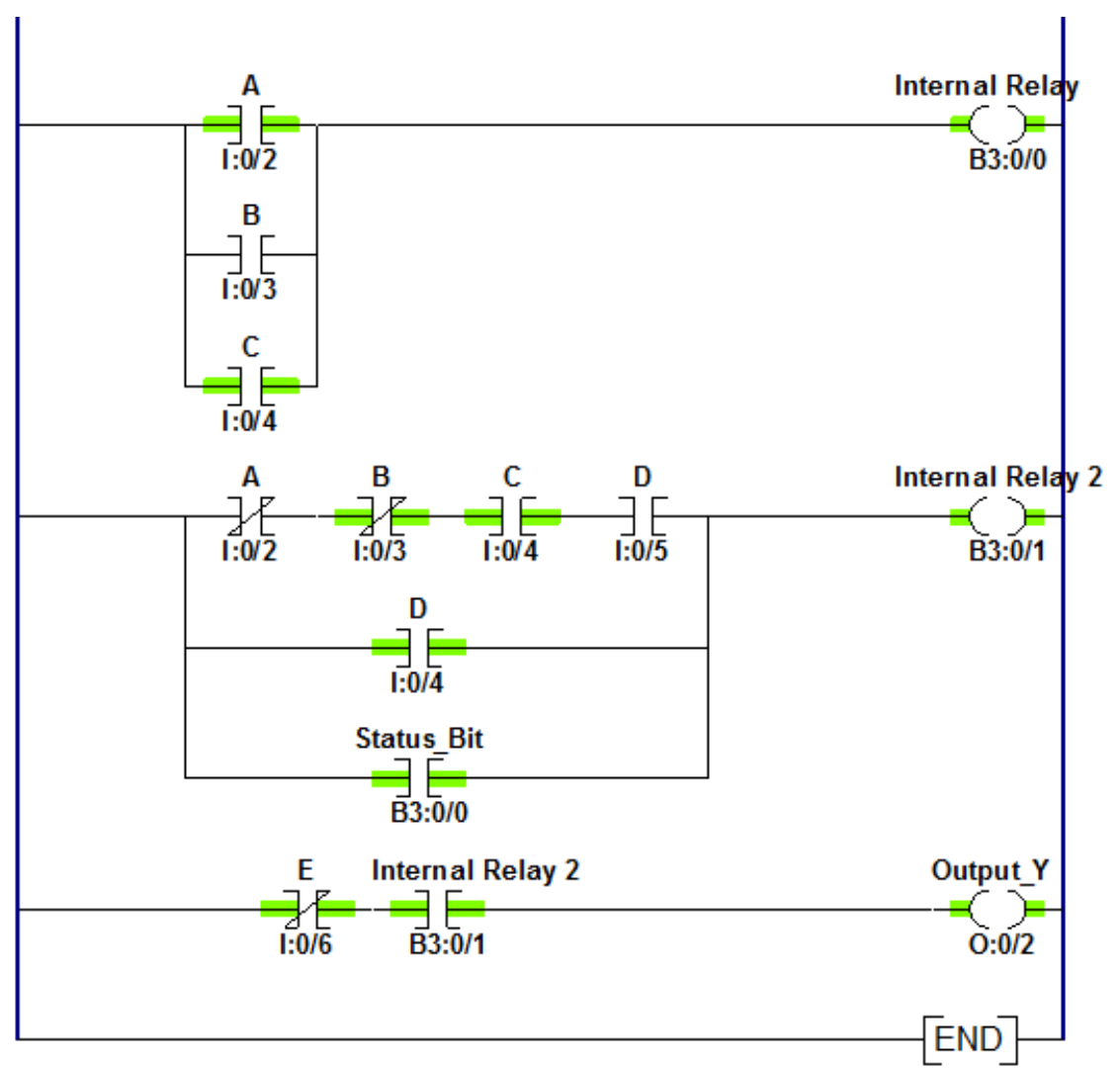

Figure 4.1: Ladder logic program for the Logic Gate Control Problem 
In Laboratory 3, students learn how to control a planer machine, a die cutting tool widely used in the industry. Although many manufacturing plants also have other refined tools such as milling machines and flatteners, planer machines are most common to smaller manufacturers due to being comparatively inexpensive and a good understanding of its operation. The machine typically uses a linear motion to move a workpiece on a table and a cutting tool above to alter and produce the parts desired.

Thus, in both labs 2 and 3, students will not only be require to review their knowledge on Logic gates but also understand the functioning of an industrial machine widely used in electronics manufacturing. They will then be required to provide the Truth table for this expression following which they will be able to identify the input conditions under which the output is turned on. Finally, this process will prepare students to simulate a working industrial machine and control it using ladder logic programming.

\subsection{Applications in the Oil \& Chemical Industry}

PLCs have found a significant role the oil and chemical industries. They are also used in pharmaceutical firms and the food industry to perform numerous operations. Common applications in these industries include batch operations for material handling, vial washing and mixing operations as well as in pump control systems. The following section is divided into 2 labs wherein students gain an understanding of how these batch operations are performed as well as how PLCs are efficiently used in these industries.

In oil and chemical applications, vial mixing and washing are important operations that needs to be carried out in a measured and systematic way. Two or more liquids are typically mixed together to form a batch wherein the level of the liquid in the tank is detected using sensors. The rate of liquid flow is fixed and the time and proportion is controlled using limit switches.

In the lab 4 application, students are provided with a control problem wherein they are required to dilute a chemical by mixing it with water in a ratio of 3:2. The mixer has 3 sensors which are depicted in the ladder programming with the use of manually operated limit switches to simulate the detection of the level of liquid in the tank. Three solenoid valves a, b and c control the inlet 
and outlet of liquid to and from the tank respectively. Once the ratio has been attained in the tank, the motor mixer begins to operate to blend the 2 liquids.

In laboratory 5, students are required to simulate a bottle filling operation that is used in mass manufacturing of liquid products. The lab was developed at the National Institute of Technology by Alok \& Goel [25]. In such mass chemical or liquid packaging operations, PLCs are often used to operate the machines or conveyor belts to detect the position of the bottles or packages following which the filling operation begins. Once the bottle has been filled, the PLC appropriately moves it along the conveyor for further sealing and labelling operations. If a manual workforce was employed to perform individual filling and packaging operations, the process can become very time consuming and tedious. This is why PLCs are used by which the process is significantly simplified and can be altered with programming to perform the desired operation.

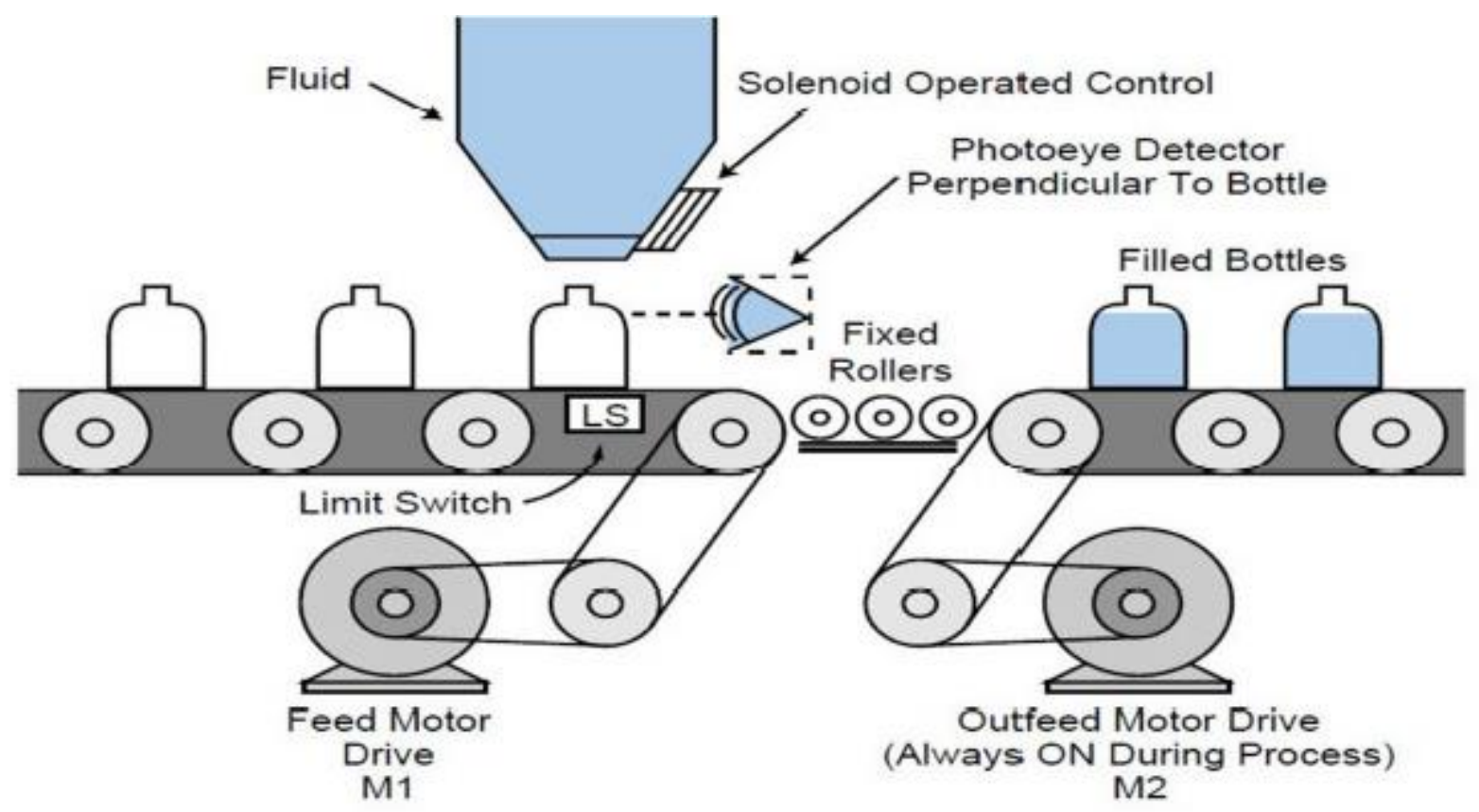

Figure 4.2: Bottle Filling Operation - Control Problem for Lab 5 [25]

Students are provided with a range of control parameters, address map and constraints in order to perform this mixer operation. Similar variations of this application is one of the most commonly 
found application in the liquid industry. This application of PLC is not limited to Oil \& chemical industry but can also be found in the food industry (soft drinks) and cleaning in industry (liquid soap, lotions).

\subsection{System Control Applications (Automotive \& Aerospace)}

In competitive industries such as Automobile and Aerospace, one of the fastest growing and increasingly demonstrated technologies is the use of PLCs. Applications of their use can be found in the manufacture of products, material handling, monitoring and data logging as well as fault detection. For such applications, PLCs are required to control multiple output devices simultaneously to perform an operation. Using PLCs to control these outputs in the desired manner and change their operation when required is what makes PLCs highly suitable for these applications.

In lab 6, students learn to control a 3 stage Air-Conditioner (A/C) system using the digital logic circuit provided to them. The A/C system consists of 2 motor compressors as well as three thermostats and a flow switch. As in the industry, students will be required to convert this electrical wiring diagram to a ladder logic program. PLCs not only simplify control problems but significantly automate processes by eliminating the need for rewiring and restructuring every time a different operation is required. If the motor compressors are to be operated at different times or the system faults need to be detected, PLCs make streamline the process i.e. we only need to alter the ladder program as opposed to restructuring and rewiring the entire physical circuitry. This is why timing and comparison constraints also play a crucial role in the successful operation of a task. This can be seen at Traffic light junctions where each light needs to be timed for a certain amount of time or in a manufacturing plant where machines need to alternate in operation to produce batches. This lab is designed to introduce students to handle these type of constraints and appropriately determine which functions and ladder programming modules to use to obtain the desired objective.

Timers, in ladder logic programming is an important tool that can significantly change the outcome or modify the operation of the task in hand based on its parameters and variables. These variables are the timer address, preset value, time base and the accumulator. Timer address is typically assigned in the T4 registry in most PLC systems. Timers also include subset addresses 
that perform different functions once activated. Students learn to use these timer modules with the series of laboratory experiments here.

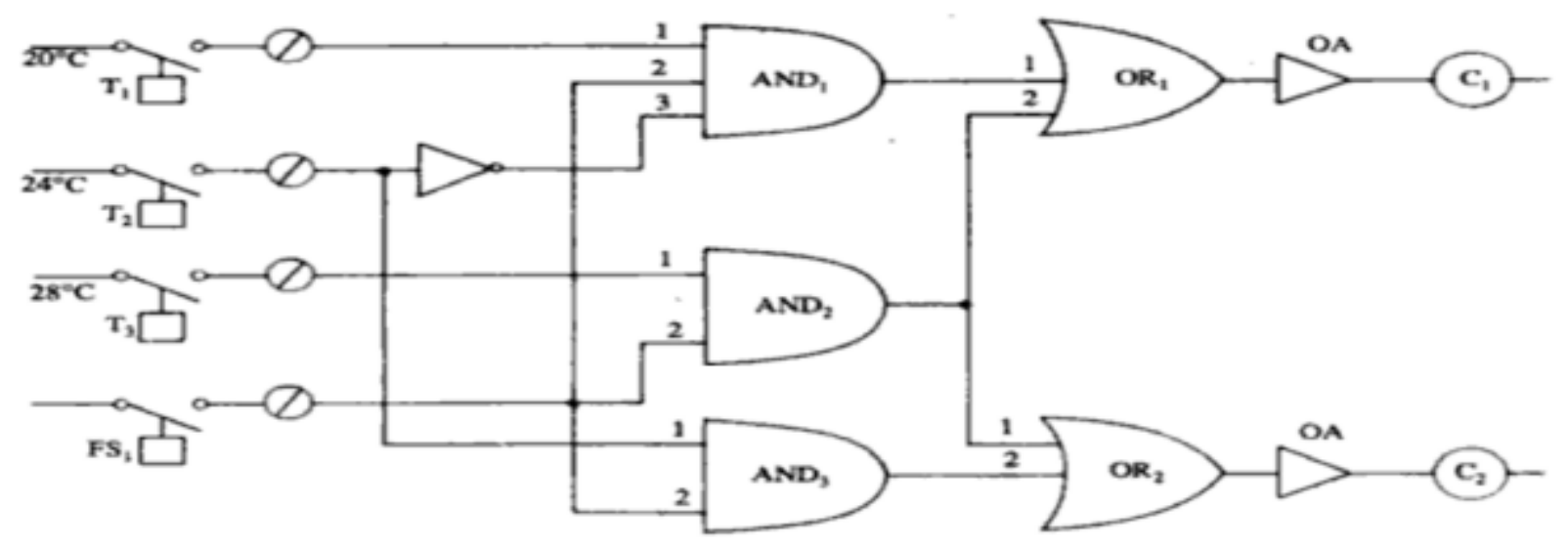

Figure 4.3: Digital Circuit Wiring Diagram for the 3 Stage A/C System (i) C1 \& C2 are the compressor motors, ii) T1, T2, T3 are the thermostats and, iii) FS1 - Flow Switch [25]

In Part 1 of laboratory 7, students are introduced to timers to control a cooling fan for 10 seconds using a single Normally-Open switch. The status of the Fan is indicated using 2 external output LEDs. As mentioned previously, timer subsets play a crucial role in handling the output fan. For example, the address T4:1.TT or the Timer Timing bit is activated only when the timer is in operational mode. Once the T4:1.TT bit is activated, any output instructions on the rung it is present on is also activated. Similarly, T4:1.DN or the Timer Done bit is activated only once the timer has completed timing and is no longer running. Using these bits along with the Timer parameters is significant to the operation and control of a task.

In Part 2 of lab 7, students are required to control a motor starter and a cooling fan by handling the constraints presented in the problem. The motor starter and fan are to operate continuously and keep running until an input switch is turned off. Long operation of the motor starter causes it to overheat and turn off. This in turn triggers a series of timer events wherein the fan is turned on only for a certain time period once the motor goes off. Thus, between certain time periods the fan continues to operate before finally turning off itself. Furthermore, during these time period when 
the motor and fan are both turned off, the switch cannot be turned back on. Finally, a reset pushbutton is to be included to reset the timers so that motor and fan can be restarted.

The timer operates similar to that as shown in Figure 4.4. Once the motor is turned off, the timer continues to operate before turning off between 5 and 10 seconds as well as beyond 30s.

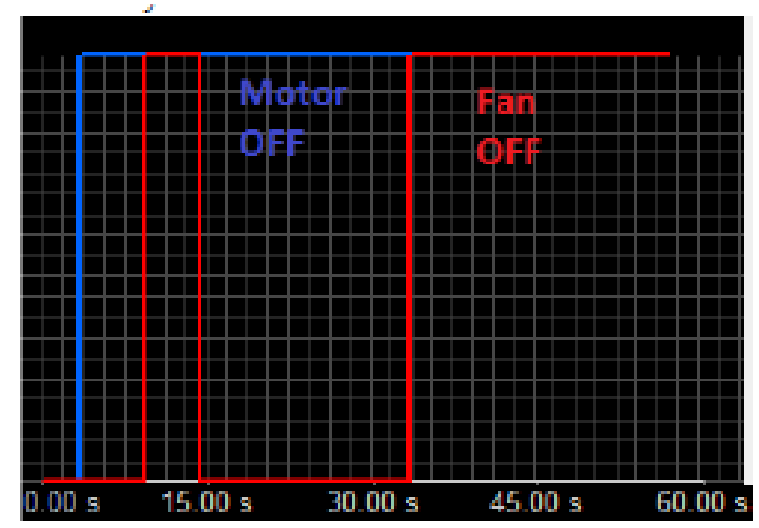

Figure 4.4: Timing Diagram for Laboratory 4. Here, the value sets to logic output 1 when the outputs are off and changes to 0 when the output is on.

The ladder logic program for this control program can be seen on the next page in Figure 4.5. Rungs 1 and 2 are used to control the Motor output to be on or off controlled by XIC and XIO input coils. It is to be noted here, that the XIO instruction for the fan is a precursor to the XIO on/off command. Thus, in the time periods that is outside the constraints for 5 to 10 seconds or beyond 30 seconds, turning on the switch again fails to turn on the motor. However, turning on the motor at other times, resets the timer to zero and the motor can operate once again. In rung 2 , these instructions are reversed and latched to turn off the motor. In Figure 4.5 (c), it can be seen that the timer instructions wherein the accumulator is outside the constraints thereby turning on the fan while the motor is still off. The status of the fan is also a pre-requisite to the rung instructions so as to ensure that motor (or timer) cannot be restarted if the fan is in the off condition while the timer is running.

However, in Figure 4.5 (b), since the timer accumulator is at 14 seconds, turning on the switch again resets the timer and turns on the motor. The preset value is also set to be for 60 seconds which when completed cannot turn on the motor or the fan thereafter. In order to restart the motor and the timer mechanism, the reset button needs to be used. 


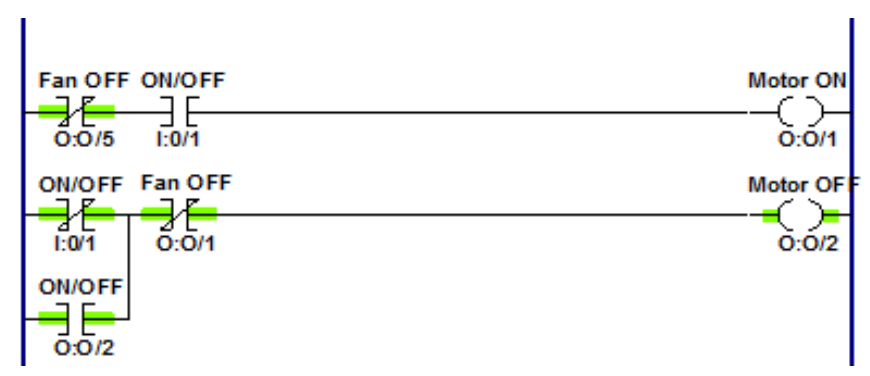

(a)

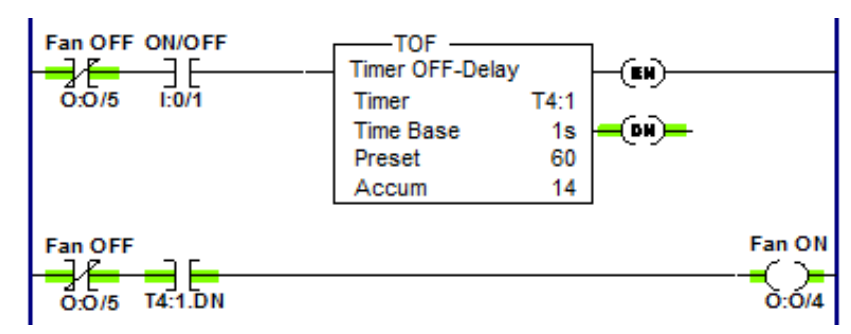

(b)

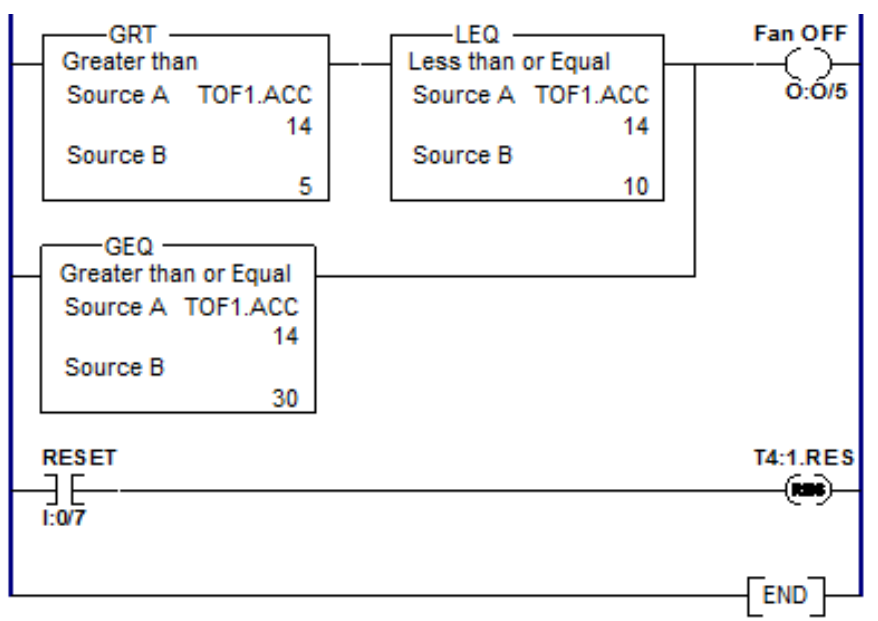

Figure 4.5: Ladder Logic Program for Part 2 of Laboratory 4 (a) Rungs 1 and 2 control the motor output (b) Rungs control the Timer OFF and the status of the fan (c) Rungs handling the constraints defined in the problem

In Figure 4.5 (c) is where the constraint is handle using comparison instructions in ladder logic programming. Thus, if the accumulator value is greater than 5 seconds or less than/equal to 10 seconds, the output (the fan) is turned off. This constraint is used in OR logic operation along with the constraint where the timer is greater than or equal to 30 seconds which also turns off the 
motor. The final rung includes the reset button and reset instruction sharing the timer address to reset the timer once the timer is complete.

The control problem designed for laboratory 8 is to control a water tank system and monitor the level of the water in 2 operational modes - Automatic and Manual Mode. The tank consists of 2 level sensors - a 'High' level and 'Low' level sensor switch which are only activated when the water level is above the high level or below the low level respectively. It is operated by an On/Off Normally-Open switch as well as a 2-position switch to change the mode of operation from Automatic to Manual. In the Manual mode, the motor pump that begins operating to remove water from the tank as long as the water level is above the 'Low' level. The status of the pump and water level is to be indicated on 3 output LEDs to demonstrate when the pump is in operation and when the water level is high or low.

Therefore, to solve the following problem we notice how it is not possible that both the low and high sensor switches cannot be activated at the same time which is where the process or interlocking needs to be implemented. Furthermore, the sensors must only function in operation when the tank system is turned on with the switch. Furthermore, the latch and unlatch mechanism needs to be incorporated in the ladder logic only when the constraints presented in the problem are satisfied.

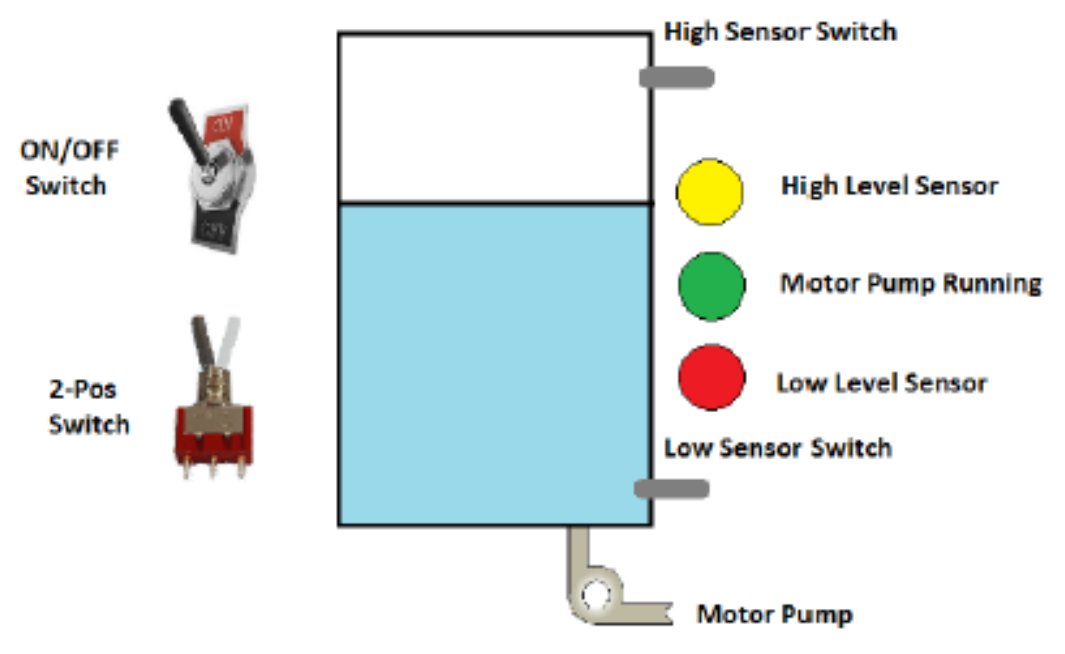

Figure 4.6: Depiction of the Water Level Monitoring Problem

In order to solve the problem, it is required to be broken down into 2 different parts. The first part is to wire the PLC input and output terminals. In Figure 4.7, it can be seen how the I/O 
terminals are connected based on all the specifications and address map provided (refer to Appendix D). Since the status of the sensor switches cannot be known unless physically wired, 2 Normally-Open switches are used which are manually closed in simulation to demonstrate when the sensor is activated and when it is not active. Herein, once the system is turned on (or when the switch is closed), the system operates in the Manual mode and the motor pump begins operation as long as the low sensor switch is activated. The high sensor level switch has no effect on the operation of the motor pump in this case as was initially desired. Turning the 2-position switch causes the system to operate in the Automatic mode.

The second part is to write a ladder logic program to control the inputs and outputs. In Figure 4.8, the ladder logic diagram is demonstrated to solve the control problem. The first rung consists of the XIC input coils to control the operation of the output motor pump. It consists of the Automatic mode, Manual mode and address to the latch/unlatch operations in Rungs 2 and 3. In this case, since the Manual mode is in operation and the low sensor switch is activated, their respective XIC instructions are turned on.
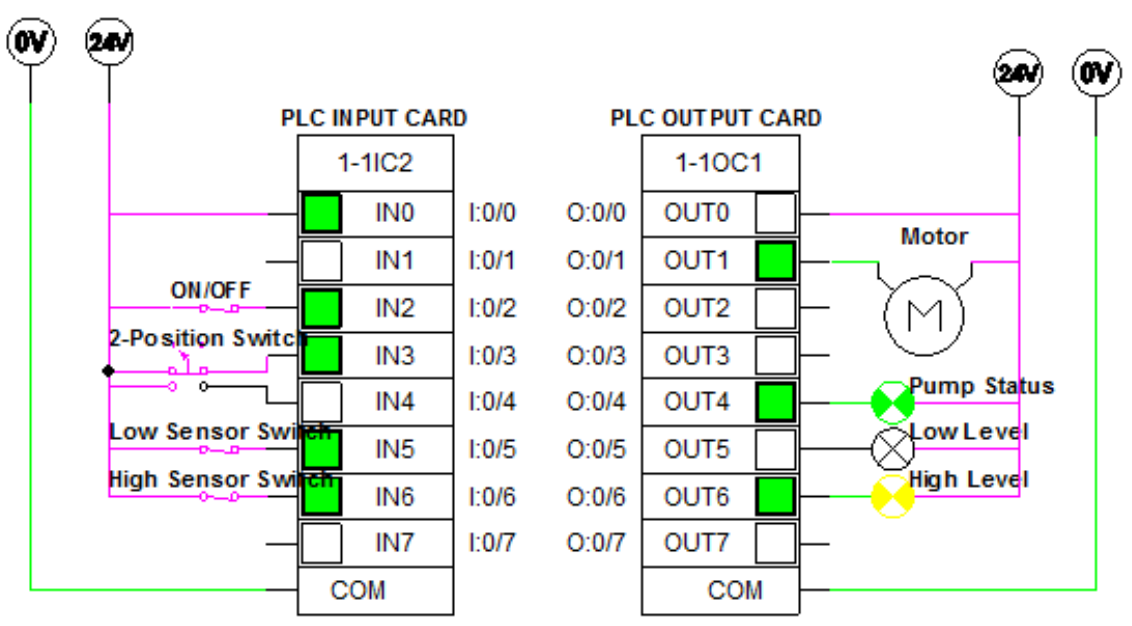

Figure 4.7: I/O Wiring Diagram on the PLC Modules 


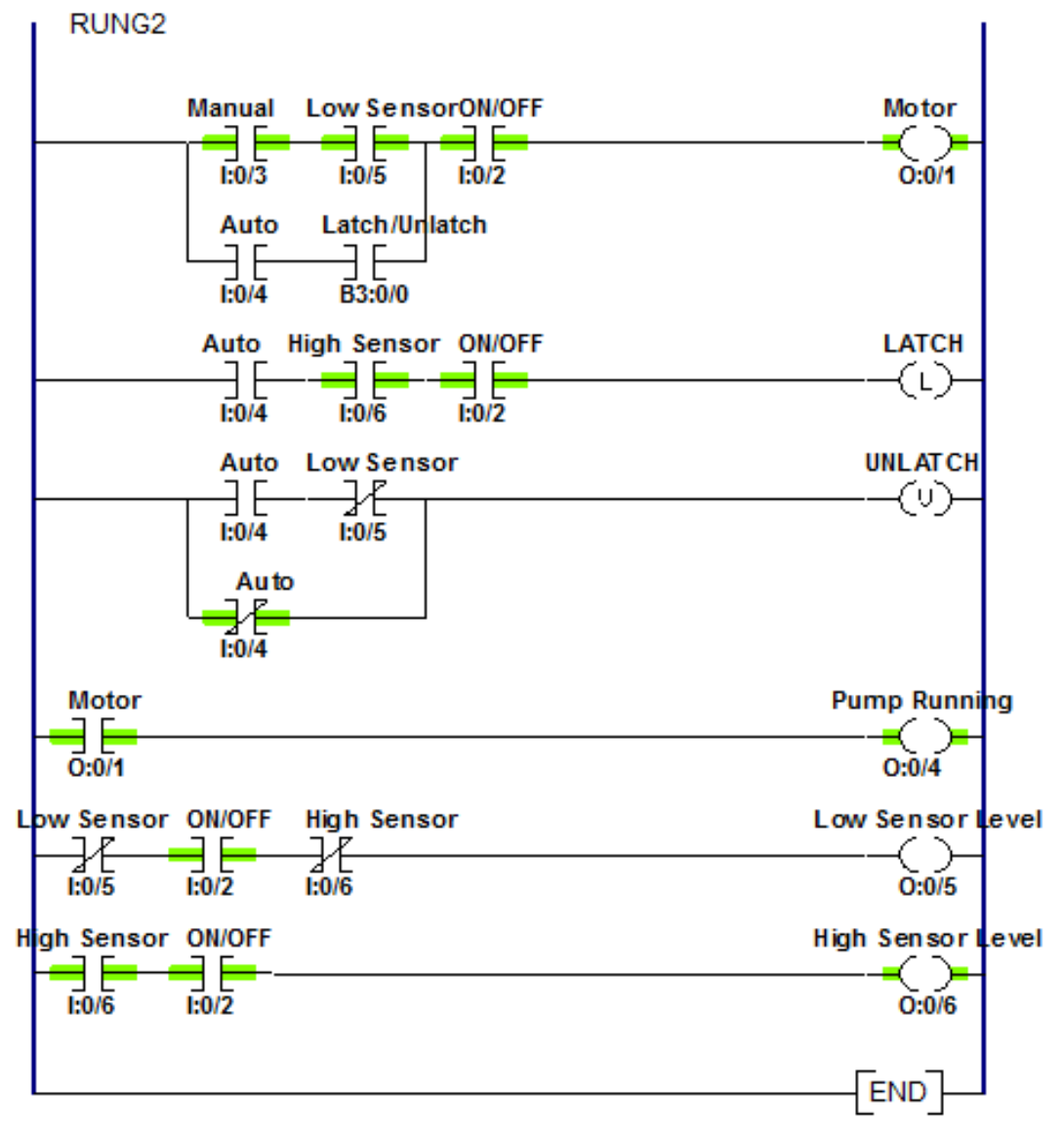

Figure 4.8: Ladder Logic Program for the Water Tank Control Problem

The rung also satisfied another constraint which was to only activate the system if the On/Off switch was turned on which is written to the extreme right of all XIC instructions. If this instruction was written at first on any of the rungs in the ladder logic program and the system was initially turned on, the motor pump would be latched on but would not stop even after the On/Off switch would have been turned off.

Rungs 2 and 3 consist of the latch and unlatch instructions to control the motor pump based on the sensor switch status. Rungs 4, 5 and 6 control the output LEDs based on the status of the Motor pumps and sensors which are written using XIC and XIO commands sharing the address of the outputs from Rungs 1, 2 and 3. 


\subsection{Applications in the Steel \& Manufacturing Industry}

Manufacturing plants employ a large number of PLCs to significantly automate processes and increase the efficiency outputs of manufactured products. Steelmaking is a multi-billion dollar industry with numerous processes, tools and techniques. This process typically begins with iron ore that is refined in multiple stages to obtain the steel from which different industries and manufacturing plants use alteration and cutting machines to produce the parts they require. Applications of their use can be found in the industry to control products conveying systems, sequencer systems, product sorting, shear press, die cutting as well as material positioning and handling. The following labs demonstrate such manufacturing operations that are commonly performed in the steel industry.

Two important concepts of Ladder logic programming are demonstrated to students in this section of laboratory experiments. The two concepts are that of 'Latch/Unlatch' and 'Interlocking'. These concepts are unique and critical to ladder logic programming. Latching or Output Latch (OTL) to a state in which the bit is turned on when the rung on which it is present is executed and continues to remain in the same state even if the rung is stopped from execution thereafter [12]. Therefore, Unlatching or Output Unlatch (OTU) is the contrary to this concept wherein the rung it is present on is stopped once it is executed. Ideally, both the instructions are used in conjunction with one another due to the fact that the only way the latch instruction can be stopped thereafter is using the unlatch instruction. Therefore, they both control the same output coil address and energize and de-energize as required.

Interlocking is a crucial component of ladder logic programming wherein two or more outputs are required to be operated but are incompatible to operate simultaneously [13]. For example, if 2 relays in an industrial machine need to be automated and operated alternatively or one at a time to prevent overheating, this concept of Interlocking can be used.

In laboratory 9, students are required to control a conveyor which is a mechanical system commonly found in all industrial plants to automate processes [5]. In this lab, students write a program the PLC to perform the conveyor operation which can be seen in Figure 4.9. The stacking system consists of 2 conveyor belts M1 and M2 along with a sensor. This sensor is used to count the transfer of each metal plate from conveyor M1 onto M2. Each time this stacking 
process occurs, the number of stacked metal plates is incremented. This is one of the most common processes while mass manufacturing of parts.

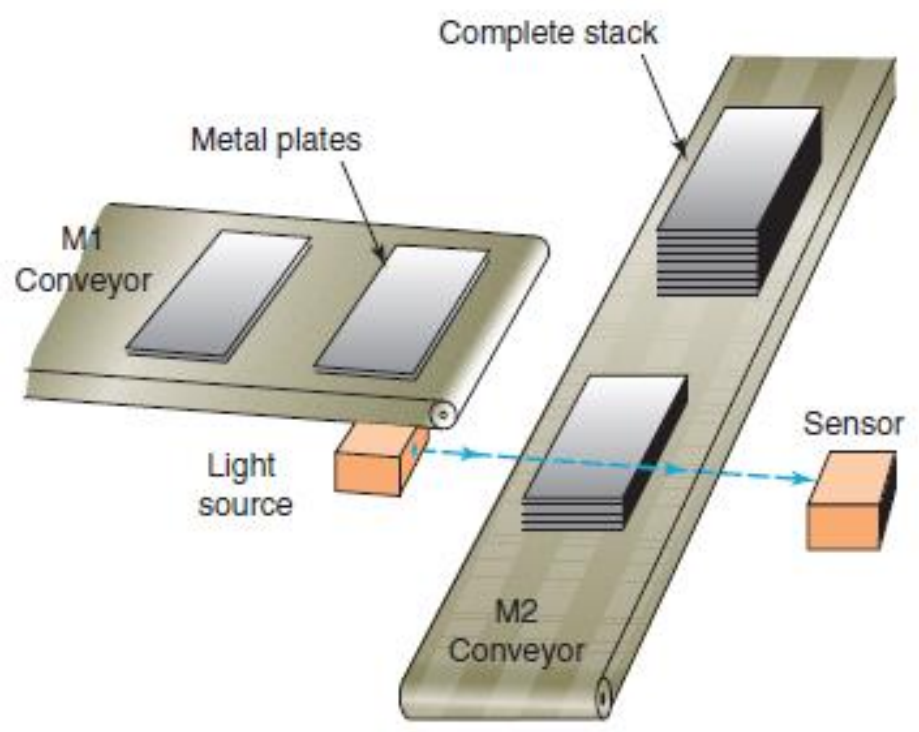

Figure 4.9: Automatic Stacking Control problem for Laboratory 9 [5]

This brings us to laboratory 10 where the control problem is to sort the manufactured parts into good and bad as they are being produced [27]. It must be noted here that the processes of cutting and sorting are not constrained to just the steel industry but is widely found in all types of manufacturing plants.

Product sorting is one such automated process that is routinely performed to distinguish good parts and bad parts. Similar to the previous laboratory, the conveyor here consists of a motor as well as a sensor. The objective of this control problem is to position the parts on the conveyor belt using the sensor in the system. In the event that the sensor detects a part, a timer is started for a period of $5 \mathrm{~s}$ following which the conveyor stops. Once the belt has been stopped, yet another timer is started for $5 \mathrm{~s}$ to restart the conveyor belt.

In order to sort the products into good or bad, gages have been incorporated in the system to distinguish between the parts and identify which ones hold defects [27]. If a good part is detected, the system continues to operate as previously - continuously detecting parts with the timer delays. In the event that the bad part is detected, the timer delay is avoided by the system 
and a pneumatic cylinder begins to operate. Students use the constraints and address map provided to them and provide a wiring diagram and ladder logic program for the same.

One of the most common operations in the steel industry is the process of die cutting, also known as Shear Press [27]. This forms the basis for laboratory 12 which can be seen in Appendix E. The process is essentially the method by which sheet metals are cut and new parts and materials are produced using shear force. Parts produced are referred to as 'Stamped' components and are a result of different shear press processes like blanking and drawing. Examples of these stamped materials or parts can be found all around us such as in canned drinks, automobile parts to household door mechanisms and other structural parts.

In this lab, students program the die cutting machine to control a solenoid cylinder that extends to stamp materials and retracts [27]. The solenoid can often be any actuator that needs to be controlled based on the type of shearing process and programming requirements. Thus, such applications are not just limited to this industry but often overlaps and can be found in variations in other industries as well.

\subsection{Applications in the Power \& Gas Industry}

Pneumatic cylinders are widely used multiple industries ranging from healthcare and automobile to oil and gas. There are numerous types of pneumatic cylinders such as air cylinders, swivel cylinders and multi-position cylinders [14]. However, this laboratory experiment is designed for students to learn about the functioning of one such type of cylinder most commonly used in air brake systems or door assist systems which typically extend and retract as desired. Laboratory is also designed to introduce students to another component of ladder logic programming which is to use counters alongside timers. The control problem here is designed requires students to incorporate various instructions and concepts from prior laboratory experiments to solve the problem. Furthermore, clocking failure events is another tool widely employed in industrial practices to record the time of failure of an operation or task which can be used to perform further analysis and improve control systems. Yet another concept introduced here is that of the 'Lockout' system which is practiced in all manufacturing facilities to lock out a system once a failure event is detected to prevent the production of defective products or any possibilities of injuries [12]. 
This laboratory requires students to control a pneumatic cylinder that is continuously operational once the system is activated and clocks the time of the event at which the circuit trips and cylinder stops functioning. If the circuit trips, it can be restarted up until three times after which the system can no longer be restarted and an alarm is raised to change the output device in order to restart the system. The control problem is bound by numerous constraints and is defined by an address map that is required to be followed which can be seen in Appendix F. The system only begins once an external input 'Reset' pushbutton is pressed after executing the program. The timing of the failure event must be clocked in hours, minutes and seconds each time the circuit trips and resets the timers if the break switch is turned on the first and the second time. Finally, the status of the circuit is indicated with an external output LED and the system is locked out only when it reaches a failure even of three times.

Figure 4.11 demonstrates the wiring connection for the Pneumatic cylinder to operate. It consists of a pilot valve, a pneumatic pressure source as well as the exhausts. Students are expected to learn about Pneumatic cylinders and understand their functioning in order to attempt this laboratory. The I/Os are defined in Appendix F which includes the 'Start' switch and a reset pushbutton to reset the timers and restore the pneumatic operation. External outputs to the PLC include an alarm, the tripped LED status and a solenoid which is linked to the pilot valve to actuate the pneumatic cylinder and begin continuous operation.

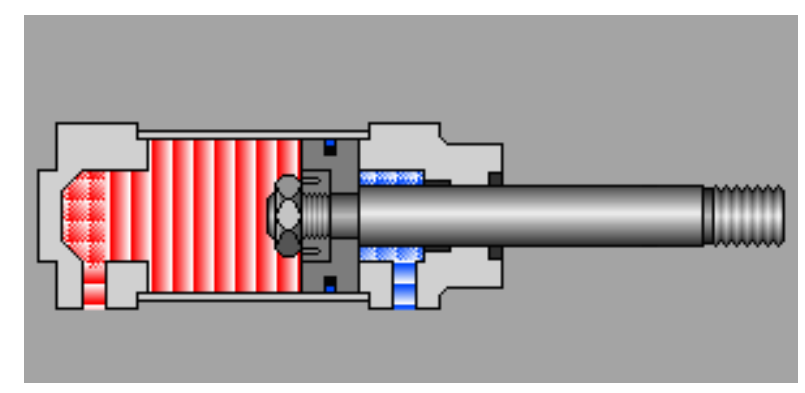

Figure 4.10: Animation of the Double-Acting Pneumatic Cylinder in Automation Studio 


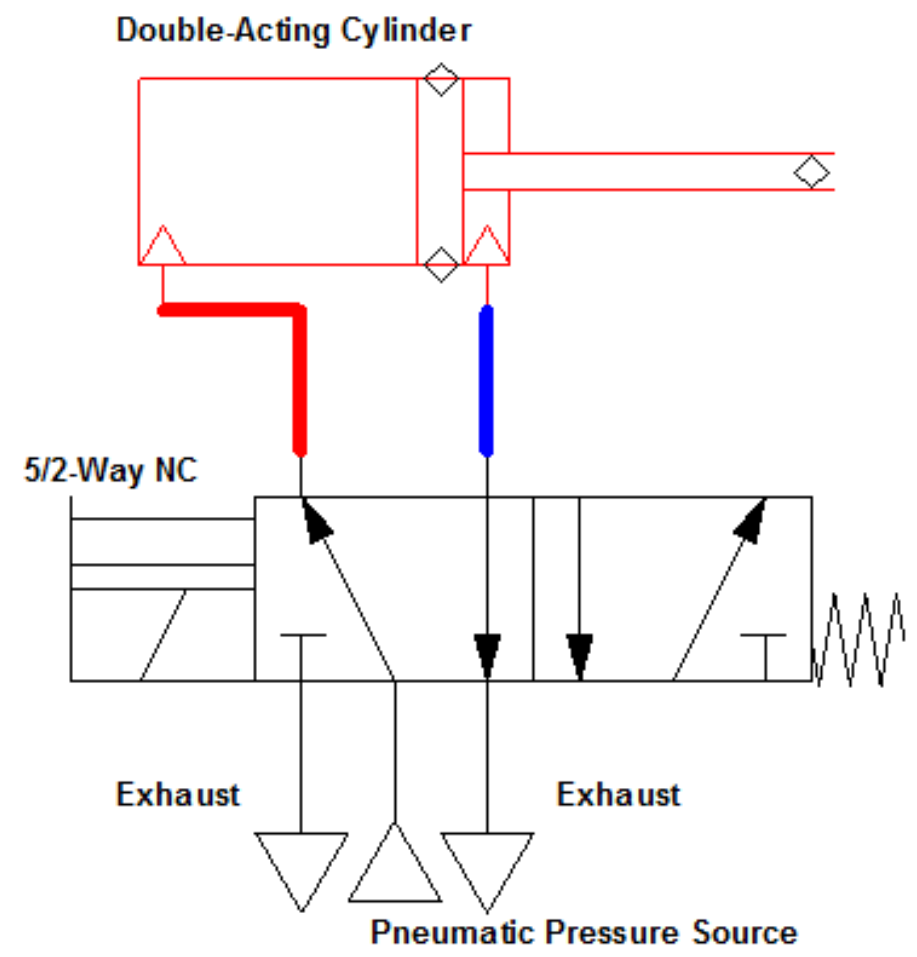

Figure 4.11: Wiring Connections to the Double Acting Cylinder to begin operation

In the next page, important sections of the ladder logic programming are presented that are critical to solving this control problem. Rungs 1 and 2 include instructions using the latch concept as well as creating an internal memory to continuously keep the timer going every 2 seconds. Thus, the pneumatic cylinder turns off every 2 seconds and turns back on which demonstrates the cylinder operating continuously. Rung 3 includes the instructions which is energized when the circuit is tripped and indicated on the output LED. The timing of the failure events is clocked using timer and the counters in Rungs 4, 5 and 6. An RTO timer is used to count the seconds and is set a preset value of 60 seconds. When the timer is done time, a counter is used to count up the minutes every 60 seconds and yet another counter is used to count up the minutes every time the minutes timer is done timing every 60 minutes. Thus, when the circuit is tripped, the timers and counters stop and the failure time can be recorded. It is critical to note here that the timers as previously use the $\mathrm{T} 4$ register and the counters use the $\mathrm{C} 5$ register which are the main address registers in most PLC modules.

In Figure 4.13, a count-down timer is used to count down to -3 times which is when the circuit must be locked out and an alarm is activated to change the device. Although, turning on the 
switch again removes the tripped status of the circuit, it fails to turn on the pneumatic cylinder and restart the program. This design also continues to indicate to the operator that there is an alarm activated and the device must be changed. Thus, this is a lockout state and the program can only be stopped completely and run again to restart the operation of the pneumatic cylinders. Other rungs can be seen in the Appendix F to reset the timers when required. Students thereby learn an important industrial operation as well as use prior laboratory skills to uniquely wire and control a pneumatic cylinder with PLC ladder logic programming.

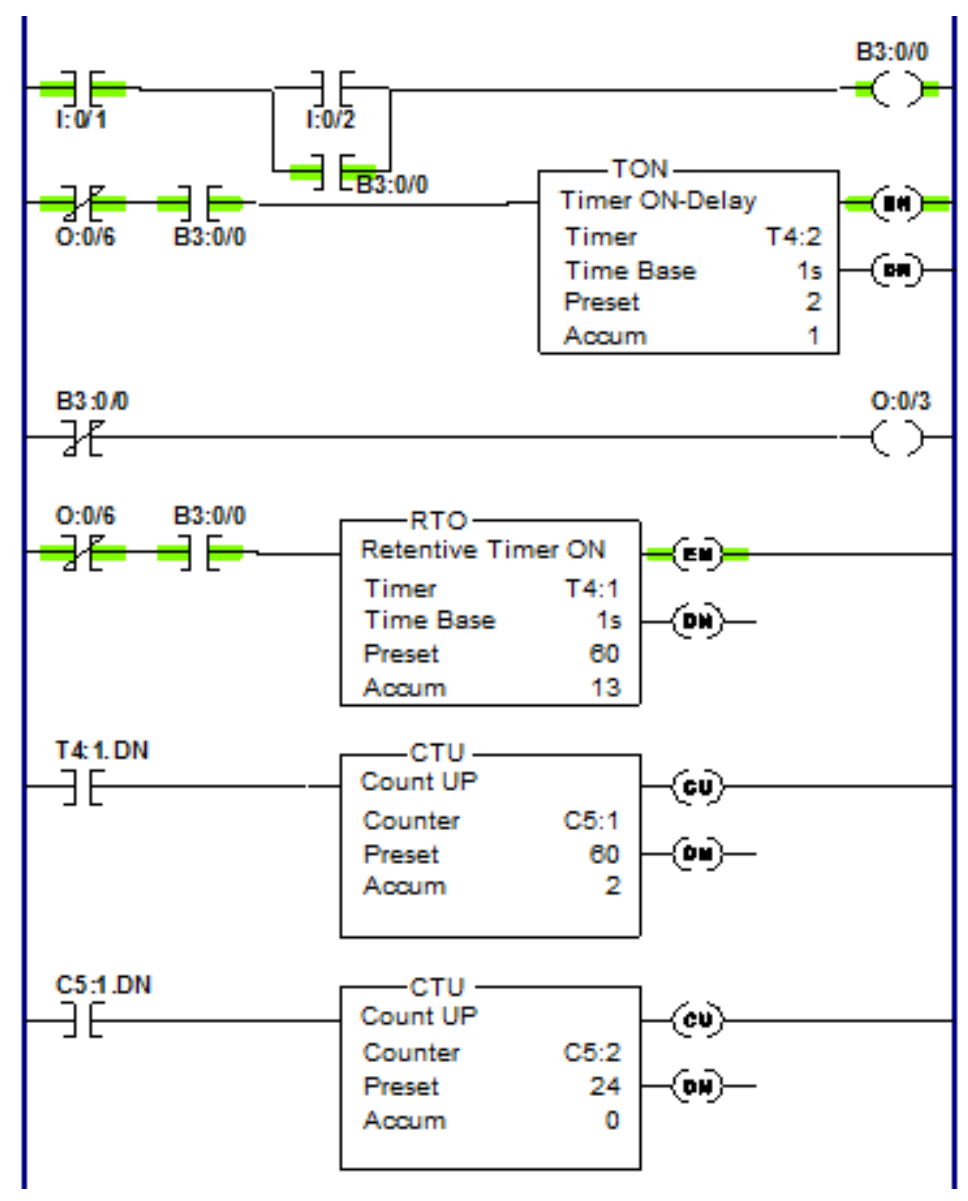

Figure 4.12: A section of the Ladder Logic Program for Lab 5 including the timers and counters that are working in conjunction with one another to clock the failure event 


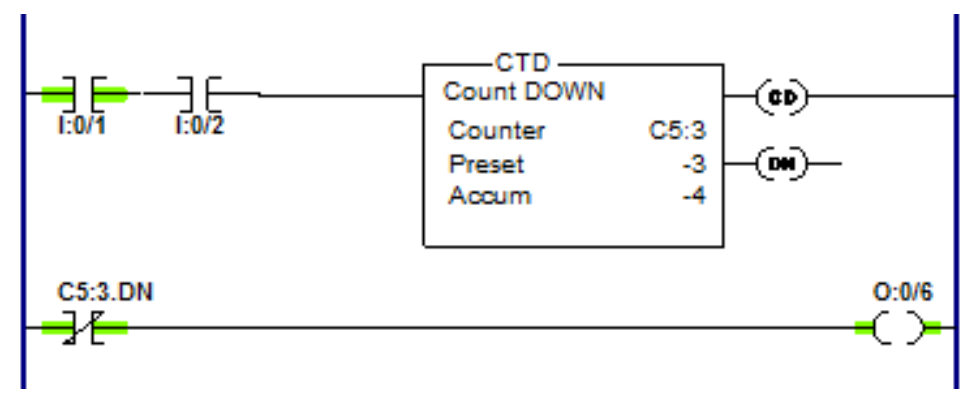

Figure 4.13: Ladder Logic program using the CTD operation which when energized leads to a lockout of the control system

Laboratory 13 is designed for students to incorporate experience from all prior laboratory exercises as well as prior knowledge to provide a solution for the complex control problem. Knowledge of motor theory is imperative to solving this laboratory. Applications of motor control are far and beyond and found in every industry from automobiles and aerospace to industrial machines and small equipment. Thus, controlling motors with PLCs is an efficient method of solving all control problems and changing operations when desired without having to modify wirings at every change operation.

The objective of this laboratory is to control the direction of a 24 VDC motor in forward and reverse directions as desired along with a coolant pump that runs simultaneously. Once the system is turned on, there is a delay of 5 seconds before which a direction of the motor can be selected. A forward and reverse pushbutton control are provided as external inputs to the PLC module. The motor must be given 5 seconds to slow down before it can change direction which must be indicated by an external LED. This is due to the fact that if a motor is operating in a forward direction, it cannot directly switch to a reverse direction but has to slow down to a stop before reversing directions and continue operation. In the event that a motor is not stopped or does not change directions for 10 seconds, the motor and coolant pump both stop. Finally, a stop pushbutton is used to stop the motor operation at any given point.

Figure 4.14 demonstrates the wiring connections for the motor using relays along with 2 indicator LEDs that are also activated based on which direction the motor is running in. This is because the software does not allow us to determine which direction the motor is operating. An H-bridge wiring connection is employed forward and reverse relays that are external outputs to 
the output module of the PLC. Once a relay is activated, the circuit is closed and current runs through the motor in the direction in which it is activated.

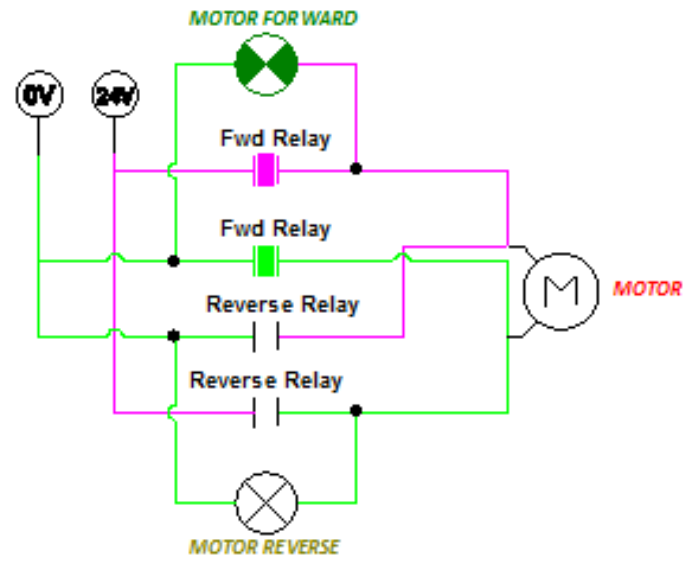

Figure 4.14: Wiring Diagram for the Motor Connection external to the PLC Module

In Figure 4.15, a section of the ladder logic programming is demonstrated of the motor running in forward direction. Rungs 1, 2 and 3 consist of instructions to operate the motor in the forward direction with XIC and XIO instructions. An internal memory bit is also used to interconnect the rungs and turn on the motor when the conditions are satisfied. Each time the motor is running in the forward direction, a retentive timer begins running for 10 seconds which is why Rung 2 ends with the instruction to check when the retentive timer is done timing.

Once the timer is done timing, the XIO coil is de-energized and the motor stops running and starts slowing down. Similarly, the rungs following these instructions perform an operation similar to that of the forward direction with the input coils reversed and another memory bit created to interconnect the rungs. Appendix F provides a complete solution the problem which includes the rungs following motor forward and reverse direction control. Each time the motor is activated in a particular direction, the output status of the motor direction controls the retentive timer. The ladder logic programming ends with a reset instruction for the retentive timer that is activated each time the motor is activated in a forward or reverse direction 


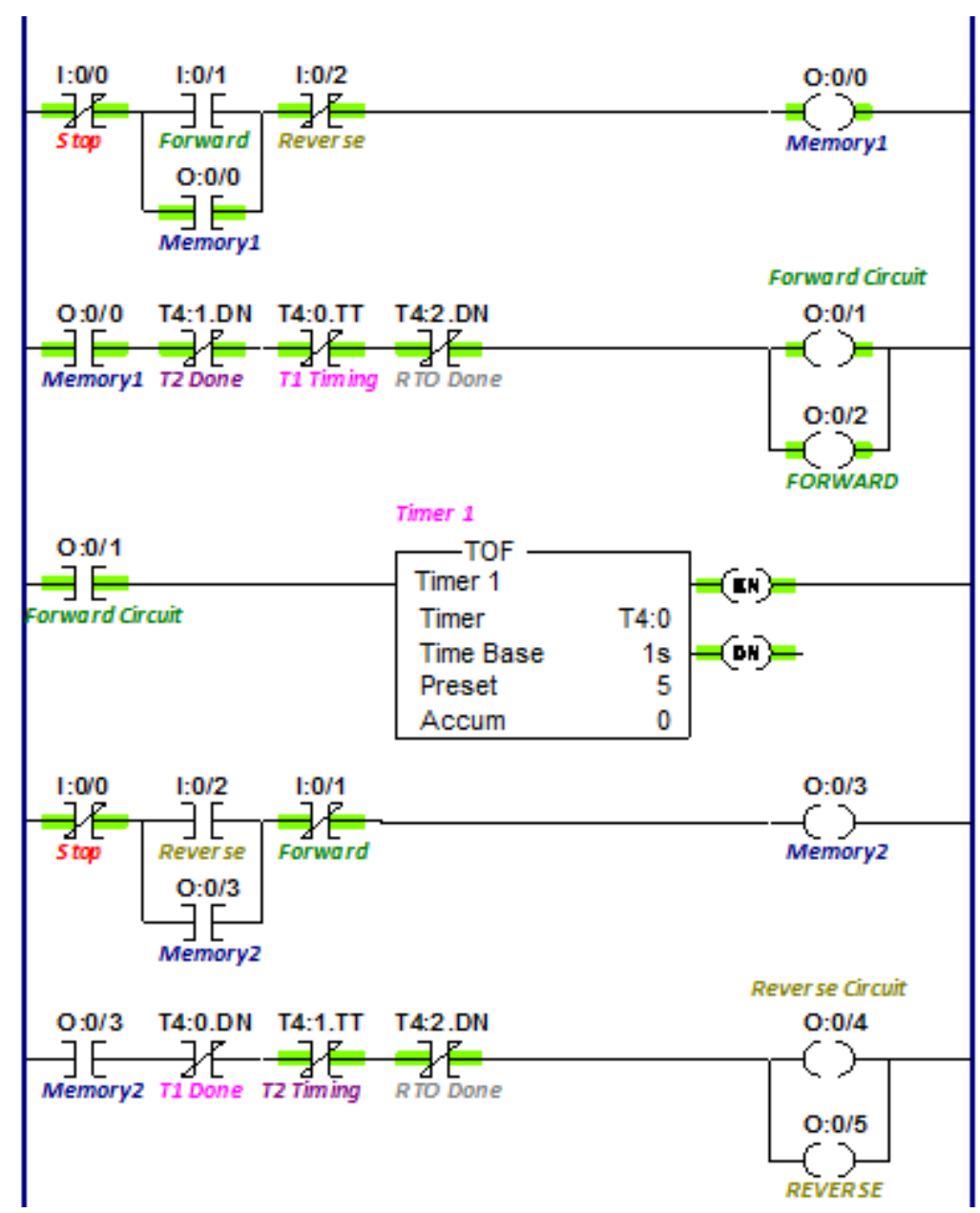

Figure 4.15: Section of the Ladder logic program in Forward Operation

Thus, the laboratory is designed for students to incorporate timers along with latch/unlatch and interlocking concepts to provide a solution to the control problem presented here. There are 2 TOF timers used here that begin timing when the motor direction is changing to indicate the slow-down status of the motor with an LED. A retentive timer is used to begin counting each time the rung instructions become true.

In the final laboratory experiment, a wind turbine control problem is presented to students. The power and electricity demands are fast increasing with the increasing industrialization and urbanization around the globe. In today's times, electricity is one of the fundamental needs of many livelihoods that needs to be sustained and developed to continue life as we know it. One of vastly developed sources of electricity generation are Wind Turbines. Over time, these sources 
have been engineered and developed to a great extent to provide us with a renewable source of electricity supply [30].

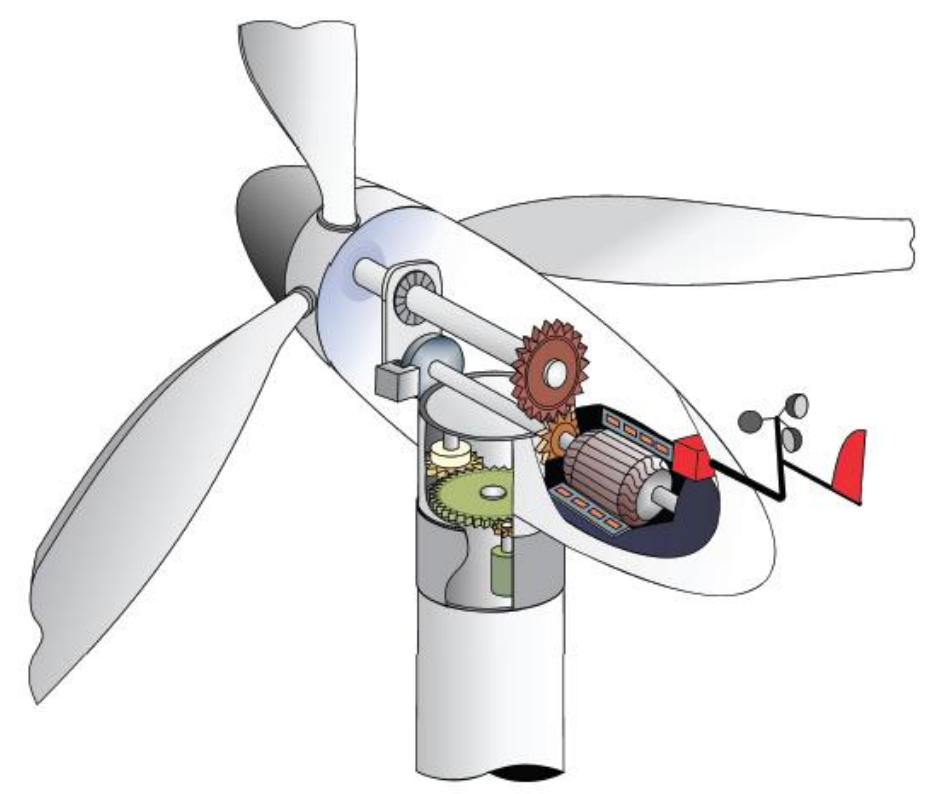

Figure 4.16: Cross-section of the nacelle present in the Wind Turbine [30]

As we know, wind turbines are devices that convert the wind energy into electricity. The varying speeds at which the wind blows causes the rotors to rotate furiously and drive a power generator inside the nacelle of the turbines [30]. The nacelle is the life source of the wind turbine that includes a control unit and other mechanical components to alter the axes of the rotors or the direction of the blades as well as monitor the functioning of the wind turbine[30]. This can be seen in Figure 4.16.

PLCs are typically used as these control units to provide the different conditions for monitoring and altering the wind turbine motors and rotor blades. In the lab, students simulate the nacelle of the wind turbine and calculate the power of the wind speed detected in the wind turbine. Another important programming functionality is demonstrated here where the solution requires students to enter into a sub-routine to calculate the power produced by the wind turbine. Again, these are bound by a series of constraints and specifications that can be seen in Appendix F.

\subsection{Validation}

The laboratory experiments designed here demonstrate the use of PLC ladder logic programming in practical industrial applications. They gradually increase in their level of 
difficulty from lab 2 to the motor control problem in lab 13 which also requires to explore the theory outside of the laboratory. In order to validate the design of the laboratory experiments, a group of 5 students were selected. The 5 students correspond to 2 third-year and 3 fourth year Electrical Engineering undergraduate students. A small questionnaire consisting of 5 questions was provided to them along with lab 2 and lab 13 from the laboratory experiments which can be found in Table 4.2. Students were required to rate their answers from 1 (low) to 5 (high). In order to attempt solving the control problems, students were divided into 2 groups - one group of 2 third year students and another group of 3 fourth year students. Students were also given a framework for laboratory 6 to make it easier for them to solve the lab.

Table 4.2: Questionnaire presented to 5 students as a part of the validation study. Students assign ranks to questions from 1 (low/easy) to 5 (high/difficult)

\begin{tabular}{|c|c|c|c|c|c|c|c|}
\hline $\begin{array}{c}\text { No } \\
\cdot\end{array}$ & Question & $\begin{array}{c}\text { Student } \\
1 \text { (3rd } \\
\text { year) }\end{array}$ & $\begin{array}{c}\text { Student } \\
2 \text { (3rd } \\
\text { year) }\end{array}$ & $\begin{array}{c}\text { Student } \\
3 \text { (4th } \\
\text { year) }\end{array}$ & $\begin{array}{c}\text { Student } \\
4 \text { (4th } \\
\text { year) }\end{array}$ & $\begin{array}{c}\text { Student } \\
5 \text { (4th } \\
\text { year) }\end{array}$ & Avg. \\
\hline 1 & $\begin{array}{l}\text { Do you have prior } \\
\text { experience with } \\
\text { PLCs? }\end{array}$ & 1 & 1 & 1 & 3 & 1 & 1.4 \\
\hline 2 & $\begin{array}{l}\text { Rate your knowledge } \\
\text { of PLCs and ladder } \\
\text { logic programming. }\end{array}$ & 1 & 2 & 2 & 3 & 1 & 1.8 \\
\hline 3 & $\begin{array}{l}\text { How difficult did you } \\
\text { find Laboratory 2? }\end{array}$ & 2 & 1 & 1 & 1 & 2 & 1.4 \\
\hline 4 & $\begin{array}{l}\text { How difficult was it to } \\
\text { solve Laboratory } 13 ?\end{array}$ & 5 & 5 & 4 & 4 & 5 & 4.6 \\
\hline 5 & $\begin{array}{l}\text { Were the laboratories } \\
\text { engaging and incite } \\
\text { your interest in the } \\
\text { subject? }\end{array}$ & 3 & 5 & 4 & 3 & 4 & 3.8 \\
\hline
\end{tabular}

On reviewing the results of the questionnaires, it was found that all the students had little to no prior experience or knowledge about PLCs besides 1 fourth-year student who had worked with PLCs during an internship work experience. All the students found laboratory 2 easier to solve whereas laboratory 6 was challenging and required more than 3 hours of time to solve. $40 \%$ of the students had to review their knowledge through an external source such as the internet or book to answer laboratory 13 and all the students had to learn or review their knowledge on 
motor theory to solve the laboratory 13 . In spite of being provided a framework solution to begin solving the problem, students found it difficult and required more than 3 hours to solve the question. Only the group 2 which consisted of the fourth-year students was able to solve the control problem in laboratory 13 . However, the feedback from the laboratory was positive and $76 \%$ of the students found that that laboratories were interesting and engaging and they would be interested in solving similar questions in the future.

\subsection{Limitations}

The validation study for this experiment is limited by the number of students that were used to perform the study. An accurate evaluation of the difficulty of the labs as well as knowledge testing needs to be performed with a larger number of subjects to perform all the laboratories from lab 1 to lab 13 to gradually understand how students progress in their understanding of PLC ladder logic programming. Furthermore, a questionnaire should also be given to students prior them attempting the lab exercises to include questions that would evaluate the technical knowledge they have before attempting the laboratory exercises. This would significantly help in understanding what aspects of the exercises need to be detailed further and whether exercises are too difficult to solve.

Finally, students are also limited by time constraints which was not taken into account in this study. Students must be evaluated while they attempt to solve the control problems to determine how long it takes them to solve each exercise to ensure each laboratory exercise is designed to be solved in a 3 hour laboratory period.

\subsection{Chapter 4 Summary}

This chapter has outlined the different laboratory experiments that were designed for third and fourth-year undergraduate students. Labs were designed with increasing complexity from an introductory lab 1 to solving complex control problems in lab 13 and lab 14. The chapter has also detailed the solutions to all the control problems and explained the learning objectives, concepts and knowledge required to solve the control problems designed. Finally, the results and limitations of a validation study conducted on 5 subject students that were required to 2 laboratory exercises has been presented. 


\section{Chapter 5}

\section{Conclusions \& Future Work}

\subsection{Conclusions}

$\mathrm{T}$

his paper has successfully presented a series of laboratory experiments to allow students to learn and test their understanding PLCs and ladder logic programming. The goal of this project was to design experiments with increasing levels of difficulty to allow students to learn about programming PLCs with practical industrial applications. Control problems presented here not just introduce students to Automation principles but also demonstrate the practical application of PLCs in industries such as electronics, automotive, power and oil and gas. Applications in these industries are demonstrated with the typical processes that occur here such as material handling and sorting, fault monitoring of motor and water systems, chemical mixing, cutting processes and power calculations amongst others. Although the 14 laboratory experiments have been validated by engaging students and increasing their interest in the subject, further study is required with larger number of subjects. Laboratory experiments introduce students to important PLC concepts such as latch and unlatch, interlocking and lock out mechanisms that are critical to solve a wide range of industrial applications. Exercises are also designed for students to use timers, counters and compare instructions to handle constraints appropriately and solve the control problems efficiently. All the laboratory exercises also provide an $\mathrm{I} / \mathrm{O}$ address map which is required to be followed to solve the problems.

With these exercises, students with no prior knowledge in industrial automation and Programmable Logic Controllers are able to solve complex control problems using ladder logic programming. The labs start with an introductory tutorial to explain the instructions, tools and concepts of ladder logic as well as the software, Automation Studio that is used to perform these exercises. The exercises not only allow students to develop programming skills but also teach them how the wiring connections are made on PLC input and output terminals. In this process, 
students also explore the theory in-depth that is required to perform the exercises providing them with a greater wealth of industrial knowledge and concepts. Finally, students develop soft skills such as critical thinking and team work by solving the control problems in teams and by using specifications and constraints provided to them.

\subsection{Future Work}

This project has presented a series of exercises to introduce students to the concepts of ladder logic programming. However, the validation study performed is not enough to aptly determine whether the laboratory exercises are an effective mechanism to allow students to learn about PLCs. Future work is required to introduce these laboratory exercises to students for one entire semester of study to gradually understand how their understanding improves and how a larger group of students find the difficulty of these laboratory exercises. In this project, the validation study only involved 5 students and they were only given 2 laboratory exercises without any time constraints. Future work needs to be done to precisely control how long each exercise takes to perform and how a larger group of students with no prior knowledge or experience with PLCs find the difficulty of these experiments. Upon evaluation of student feedback, more complex ladder logic exercises can be developed if necessary involving the use

of concepts of subroutine and program control instructions. Similarly, if required exercises can also be simplified providing further details and reducing the number of constraints to make it easier for students to solve the problems in a 3 hour laboratory class.

Moreover, this project can also be used as a framework to setup a physical PLC trainer module including a PLC module along with I/Os such as pushbuttons, switches and output LEDs. Although the concepts of ladder logic programming would still be the same, a practical exercise such as this would require students to manually wire the PLC I/Os as well as engage them in manually playing with the inputs and visualizing the outputs in front of them as opposed to simulating these exercises. 


\section{Appendix A: Introduction Laboratory One: Ladder Logic}

\section{Programming}

\section{A.1 Overview}

Automation Studio is design and simulation software developed by Famic Technologies Inc. primarily for the use of training and troubleshooting electrical control systems and learn about PLC ladder logic [11]. Each lab in this manual has been designed to uniquely learn about different components of Ladder Logic by solving industrial control problems to give students a wholesome learning experience.

Lab 1 begins with a tutorial and background on the critical components that will be required to solving the control problems as well as tips on how to navigate through Automation Studio. These components are to be used as a reference while solving the laboratories thereafter.

Beginning Lab 2, students will answer different control problems and unique present solutions based on the specifications and constraints provided to them. Prior to each lab, students are expected to review their knowledge from prior studies or lectures as well as the contents of the lab.

\section{A.2 Background}

PLC Programming can be defined by various programming languages such as Functional Block Diagram, Structured Texts and Instruction Lists. For the laboratories designed here, the Ladder Logic programming method has been employed which we will learn about now. 


\section{A.2.1 Basic Instructions}

In order to solve any control problem through ladder logic, input and output instructions are common to all programs which when used appropriately can perform a task efficiently and quickly.

These include the Examine If Closed (XIC) Examine If Open (XIO) and Output energizer (OTE). These instructions when used in combination with one another can be used to perform Boolean logic operations [5].

Figure A.1 demonstrates a simple rung instruction consisting of a Normally Open and Normally Closed contact in the 'AND' logic operation which when true, energize the output Y.

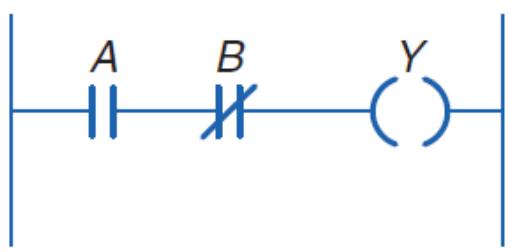

Figure A.1: Basic Ladder Logic Rung Instruction [5]

Similarly, ladder logic includes a wide range of input and output instructions that can be critical to performing key tasks.

\section{A.2.2 Timers}

Before beginning this lab, it is important to understand the types of timers and how they function. Timers are an integral part of PLC Ladder logic that are widely used to solve control problems with timing constraints. There are 3 types of Timers used in PLC ladder logic which are:

i) $\mathrm{TON}-\mathrm{ON}$-Delay Timers

ii) TOF - OFF-Delay Timers

iii) RTO - Retentive Timers 


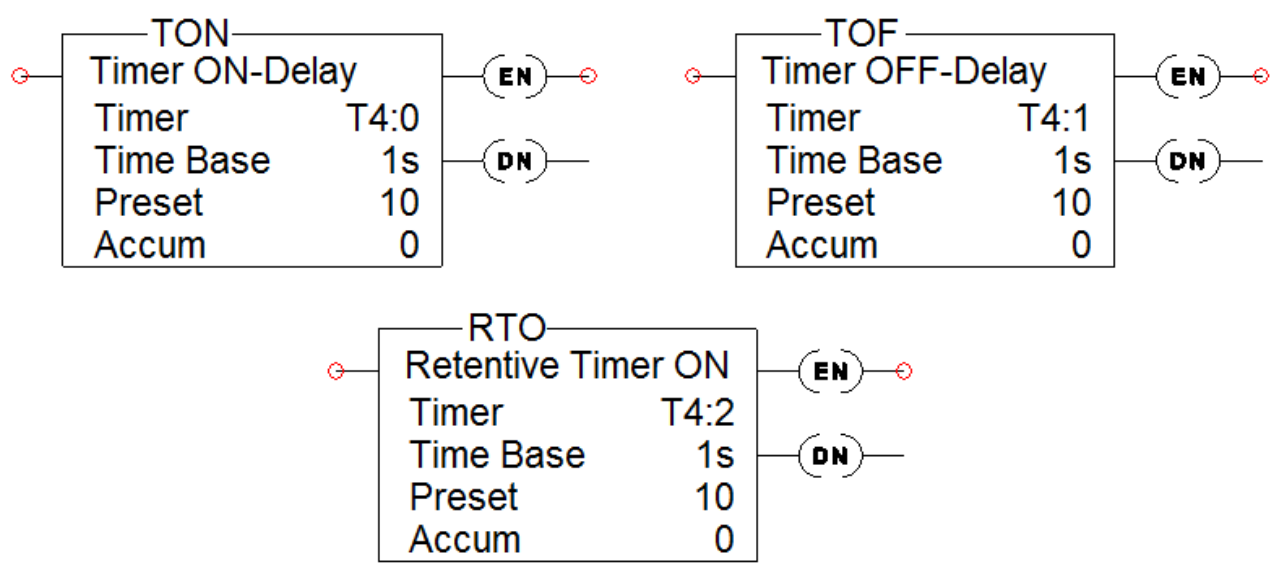

Figure A.2: Types of Timers use in PLC Ladder Logic [15]

Timers are used in a wide variety of applications. Typically, they are used to delay actions, perform an action for a limited period of time or to accumulate the amount of time that has accrued over the course of an action.

Figure A.2 displays the types of timers used in ladder logic. However, it is crucial that these timers are programmed appropriately by the programmer to achieve the desired outcome. Each timer is programmed by defining its variables such as the Time Base, Timer Address and Preset.

i) Timer: This refers to the address that the timer belongs to and is defined in the format - $\mathrm{T}: 4 / \mathrm{N}$, where 4 refers to the address for all Timer instructions (in most PLCs) and $\mathrm{N}$ is an integer which is a unique identification number of the specific timer instruction [15].

ii) Time Base: It is the interval the timer uses to function which can be set at a value between 1 second and 0.001 second.

iii) Preset: This is one of the main variables that defines a programmer's application which defines how many intervals to count before the timer should stop.

iv) Accum: The accumulator is the number that displays the count value while the timer is in progress. 
An important and useful attribute of all timers is their Status bits which help us identify the state of the timer. They have a similar characteristic in TON and RTO timer but differs in TOF timers which is indicated in parenthesis below.

i) T4:N.EN - Timer Enabled Bit: Bit is set to HIGH when the timer's rung is TRUE (FALSE for TOF timers), Bit is set to LOW when the timer's rung is FALSE (TRUE for TOF timers) [15].

ii) T4:N.TT - Timer Timing Bit: As a timer begins and the rung is TRUE (FALSE for TOF timers), and the accumulated value remains below the Preset value, the bit is set to HIGH [15]. A simpler way to understand this is - the bit is set to HIGH when the timer is $\mathrm{ON}$ and set to LOW otherwise.

iii) T4:N.DN - Timer Done Bit: The DN bit is set to HIGH when the timer is done counting and the timer's rung is TRUE (FALSE for TOF timers), it is set to LOW in all other cases [15].

\section{A.2.3 Counters}

PLC Counters are one of the most important tools of ladder logic programming.

Using Counters, like timers can be a challenging to use efficiently and for the desired application. There are 3 different types of counter instructions which are:
i) $\quad$ CTD - Count-Down Timer
ii) $\mathrm{CTU}-$ Counter-Up Timer
iii) $\quad$ RTO - Retentive Timer 
Table A.1: Description of different types of Counters used in Ladder Logic [15]

\begin{tabular}{|c|c|c|}
\hline Instruction & $\begin{array}{c}\text { This instruction is } \\
\text { used to }\end{array}$ & $\begin{array}{c}\text { Functional } \\
\text { description }\end{array}$ \\
\hline Count Down & $\begin{array}{c}\text { Count dorn from a desired } \\
\text { value to zero }\end{array}$ & $\begin{array}{c}\text { An operator interface display shows the } \\
\text { operator the number of parts } \\
\text { remaining to be made for a lot of,say 50 } \\
\text { parts ordered. }\end{array}$ \\
\hline Count Up & Count from zero up to a \\
desired value & $\begin{array}{c}\text { produced during a specific work shift } \\
\text { or batch.Also counting the number of } \\
\text { rejects from a batch. }\end{array}$ \\
\hline High-speed \\
Counter & $\begin{array}{c}\text { Count input pulses that are } \\
\text { too fast,from normal input } \\
\text { points and modules }\end{array}$ & $\begin{array}{c}\text { Most fixed programmable logic } \\
\text { input points that allow interface to } \\
\text { high-speed inputs. Signals from an } \\
\text { incremental encoder would be a typical } \\
\text { high-speed input. }\end{array}$ \\
\hline Counter Reset & To reset a counter or timer & $\begin{array}{c}\text { Used to reset a counter to zero so that } \\
\text { another counting sequence can begin. }\end{array}$ \\
\hline
\end{tabular}

Similar to Timers, counters also have an addressing format. All counters addressed in the C5 register of the PLC address book, although this may differ with some manufacturers. The different types of bits are as follows:

i) C5:13/DN - Counter Done Bit: Bit is set to HIGH when the counter's accumulator value is equal to or greater than the preset value. The value 13 refers to bit 13 which is known as the Done bit [15].

ii) $\quad$ C5:14/CD - Counter Down Bit: The bit refers to the count-down enable bit which is set to HIGH when the rung instructions it is present on becomes True. 
iii) C5:15.CU - Counter Up Bit: The count up bit is set to HIGH to count up from a zero value to a desired preset value. Again, the counter only beings counting when the rung it is present on becomes True.

\section{A.2.4 Automation Studio}

Here are some tips to navigate through Automation Studio to complete the lab procedures.

All PLC components as well as electronic components can be found the Library Explorer window. This can be seen on the toolbar at the top with the icon -

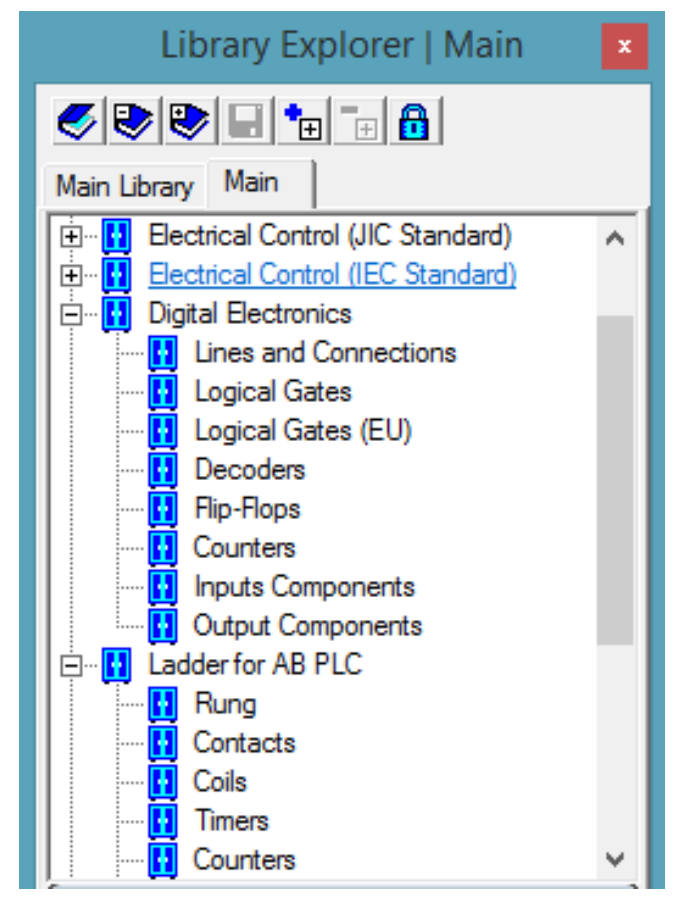

Figure A.3: Library Explorer on Automation Studio [11]

In order to change Timer parameters, change the address and configuration of the Timer, import the Timer to the drawing and double-click on the timer to open up the box as below. Click on Modify to change the Preset value as desired. 


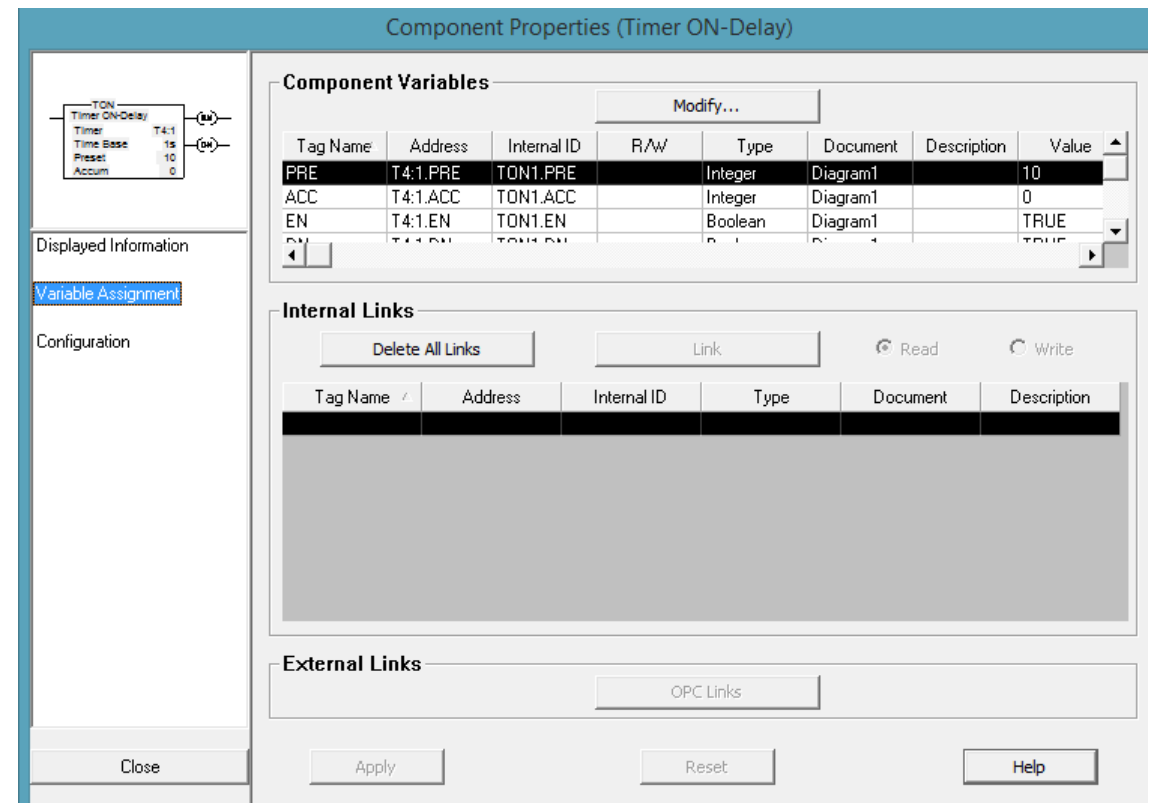

Figure A.4: Component Properties (in order to change Timer Properties) [11]

To view, add and manage variables, you can use the variable manager to ensure all your parameters are as desired. This can be found on the toolbar at the top with the icon - $x_{1}^{\prime}$

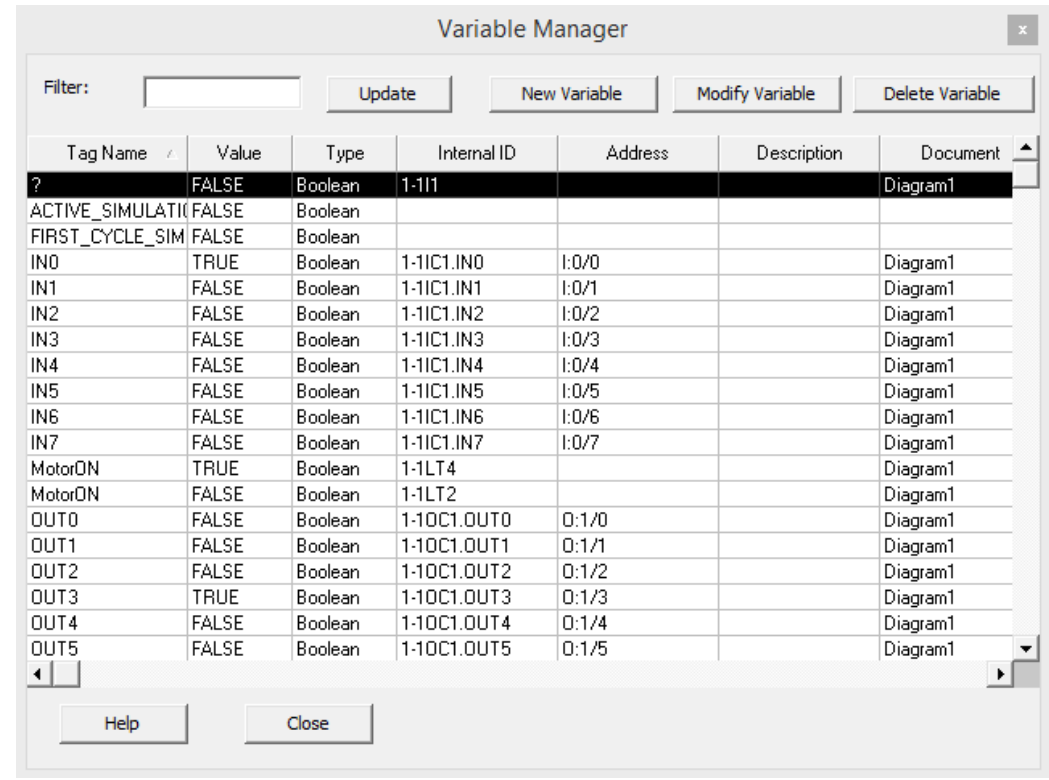

Figure A.5: Variable Manager (to add, delete, modify \& manage variables) 


\section{A.3 Laboratory Procedure}

\section{A.3.1 Part 1}

Wire an 8 Input PLC Module along with address in the PNP and NPN wiring connections.

\section{A.3.2 Part 2}

Convert the following Structured Text program to a ladder logic program and answer the questions that follow.

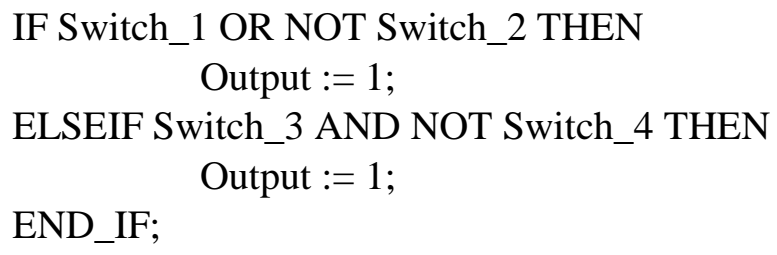

i) What is the status of the Output when Switch 2 and Switch 3 are closed?

ii) If Switch 2 is opened, does the status of the output change?

iii) Is the output energized if all the input switches are open?

\section{A.3.3 Part 3}

As an Electrical engineer, you have been contracted by an Automobile company to control 2 Output indicator lights, Output $\mathrm{X}$ and Output $\mathrm{Y}$ using a single Input Pushbutton. You are required to actuate the outputs using 2 different methods of input instructions. A flashing coil that is continuously operating which must turn OFF when the input pushbutton is pressed.

Table A.2: I/O Address Map for Problem A.3.3

\begin{tabular}{|c|c|c|}
\hline Address & Type & Description/Functional Requirement \\
\hline $\mathrm{I}: 0 / 1$ & External Input & Input Pushbutton \\
\hline $\mathrm{O}: 0 / 2$ & $\begin{array}{c}\text { External } \\
\text { Output }\end{array}$ & Indicator Light (Green) - Output X \\
\hline $\mathrm{O}: 0 / 4$ & $\begin{array}{c}\text { External } \\
\text { Output }\end{array}$ & Flashing Coil \\
\hline O:0/6 & $\begin{array}{c}\text { External } \\
\text { Output }\end{array}$ & Indicator Light (Red) - Output Y \\
\hline
\end{tabular}

Use the JMP and LBL instructions to turn on Output Y. 


\section{A.4 Post-Laboratory Requirements}

Students are expected to hand in their lab reports individually or in groups of 2 by the next laboratory class to the lab TA. Your lab report must include the following sections -

i) Standard Electrical Departmental Cover Page

ii) Introduction

iii) Results \& Discussion (reasoning behind your logic design must be explained in detail along with screen captures of the design)

iv) Conclusion

\section{A.5 Laboratory One - Solutions}

\section{A.5.1 Part 1 Solutions}

The connection of Sink/Source in PLC wiring is one of the fundamental principles that is critical to the proper functioning of a PLC module. PLC I/O Modules must be appropriately powered using a voltage supply and common ground.
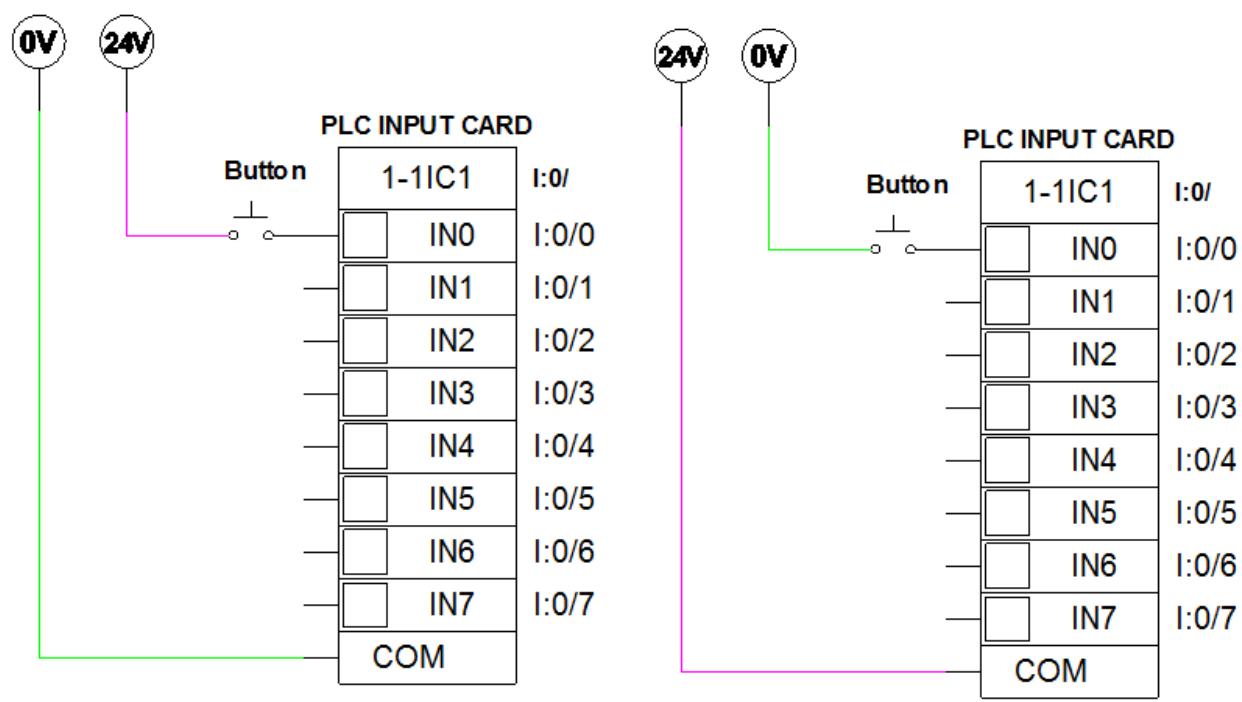

Figure A.6: PLC Input Module connections when NPN sensors are being used (left) and when PNP sensors are being used (right). In the case of NPN sensors, applying 0 VDC to the common terminal prevents the PLC from sensing the input which is why the connection is reversed 


\section{A.5.2 Part 2 Solutions}

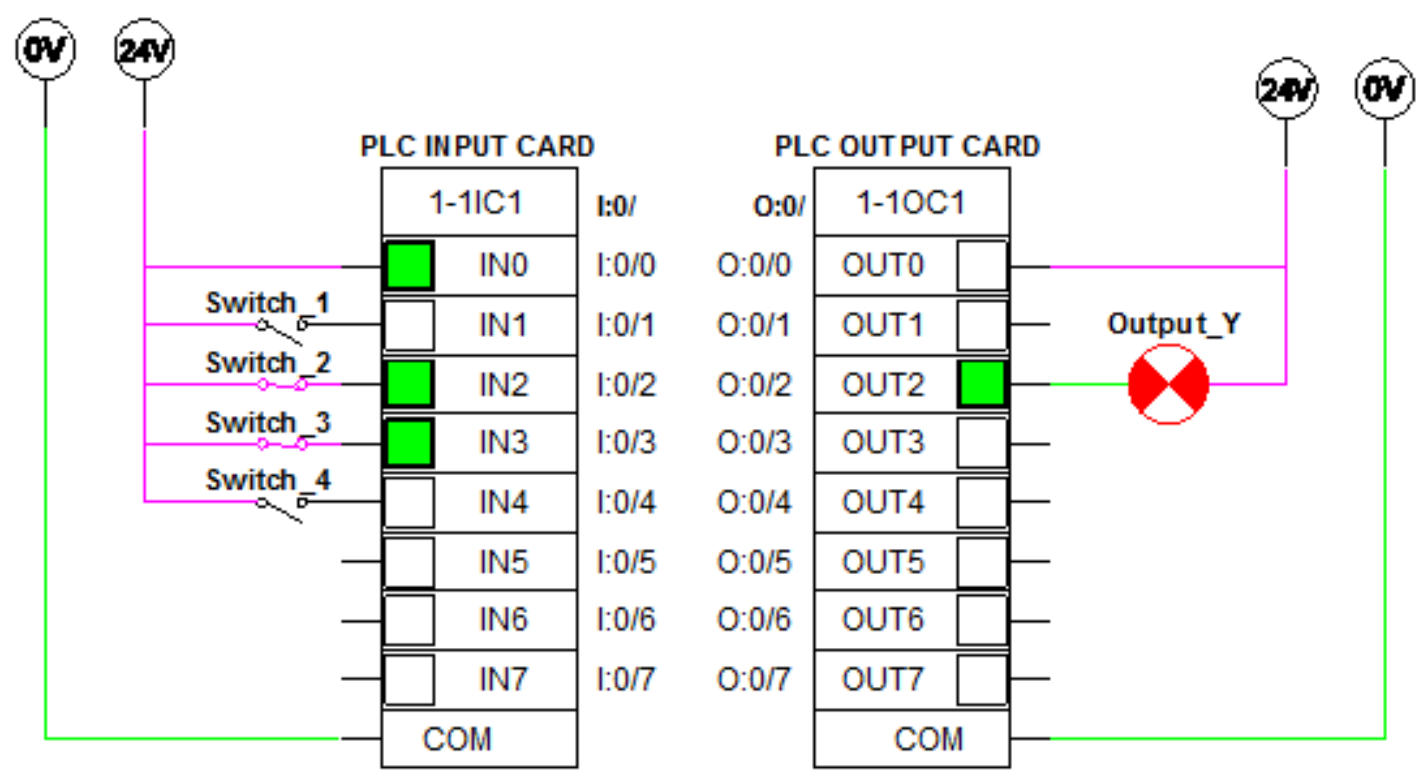

Figure A.7: I/O Wiring for Part 2

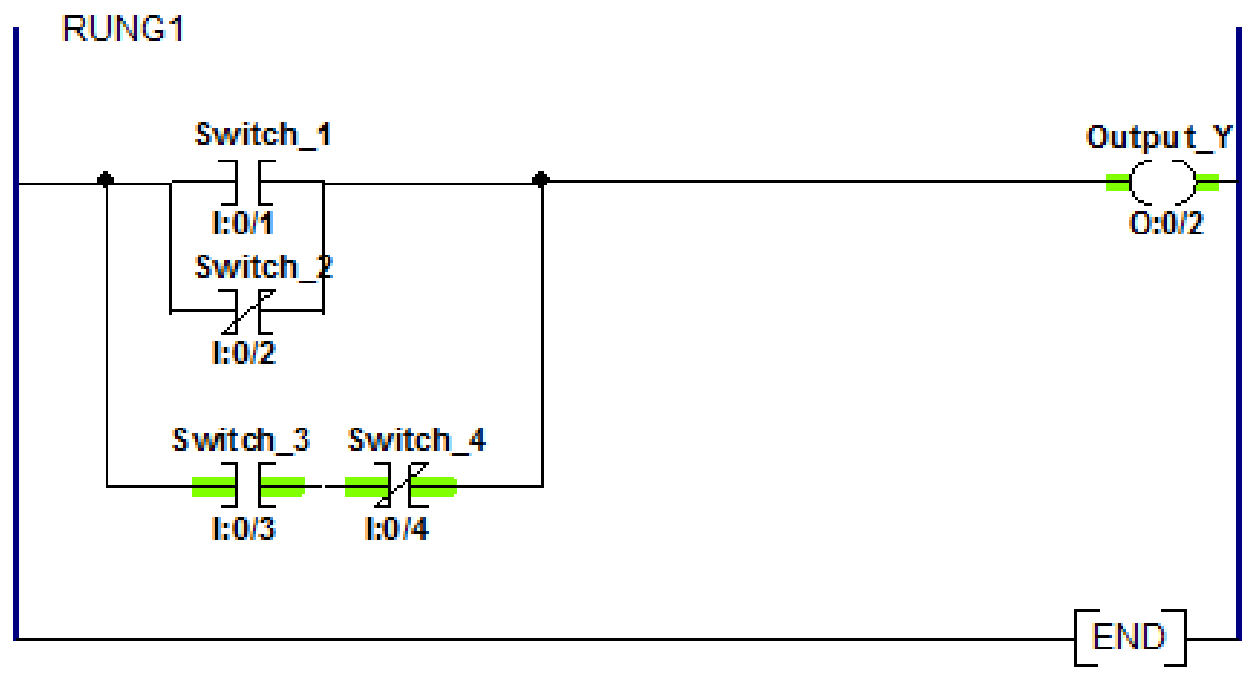

Figure A.8: Ladder Logic Diagram converted from Structure Text 


\section{A.5.3 Part 3 Solutions}
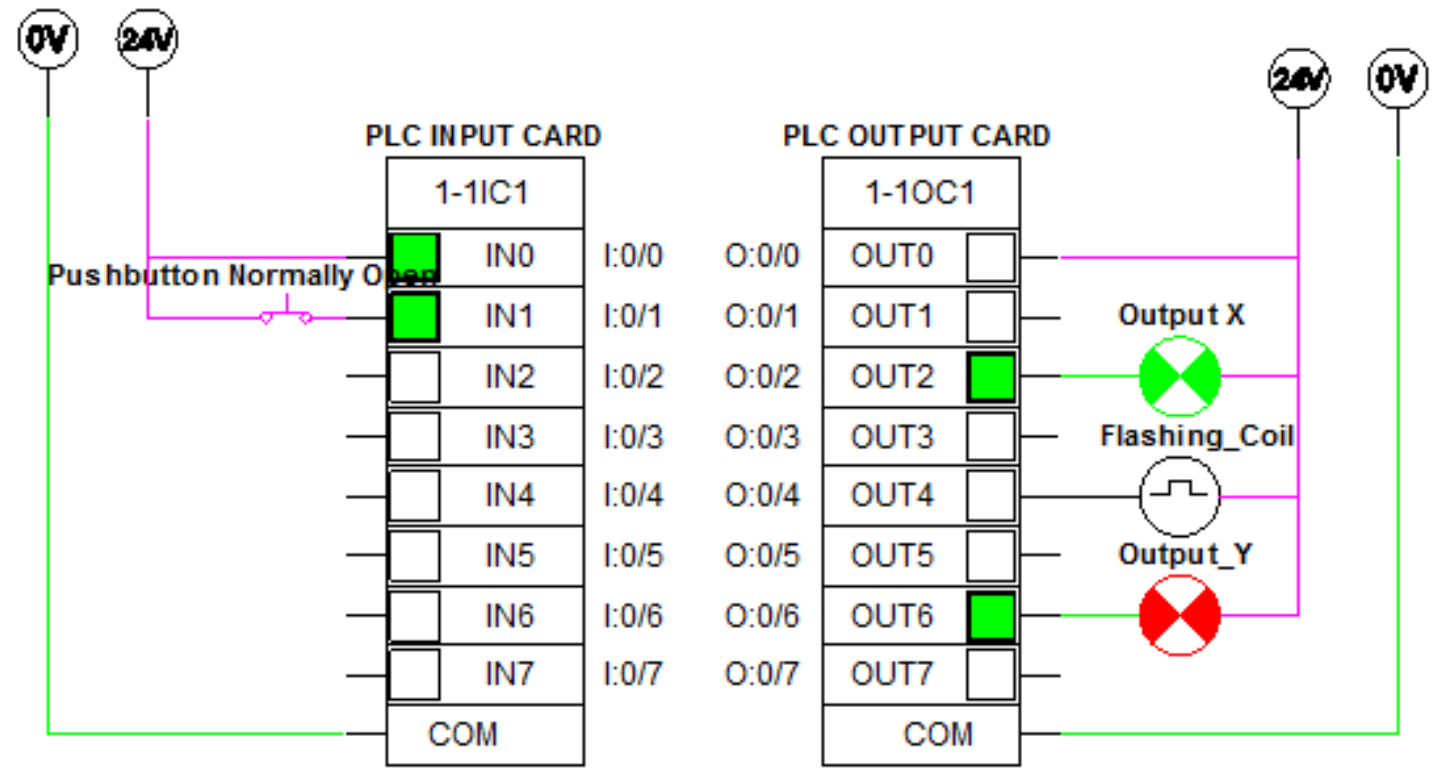

Figure A.9: PLC I/O Wiring Diagram for Part 3

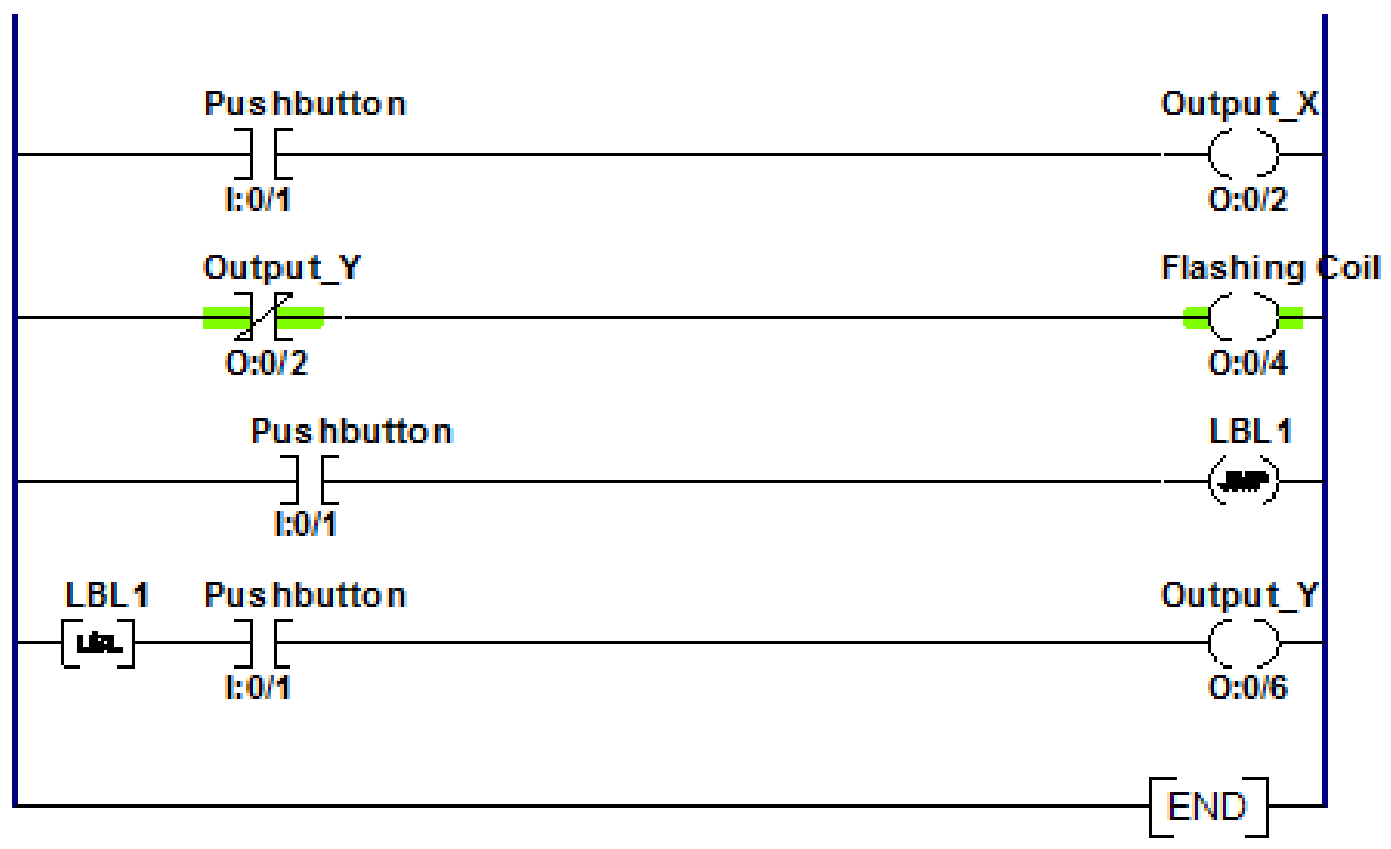

Figure A.10: Ladder Logic Diagram for Part 3 


\section{Appendix B: Applications in Electronics \& Avionics Industries}

PLC digital systems can be designed using logic operators like OR, AND and NOT functions. An understanding of their functionality and operation in combination with one another can be useful to solve various control problems. In the field of Electronics \& Avionics, PLCs are widely used for the manufacture of Printed Circuit Boards and other parts for material handling, inventory control as well as in product conveying systems.

In the following 2 labs, students use ladder logic to perform ladder logic operations as well as learn how to perform a planar machine control - a system widely used in manufacturing operations.

\section{Laboratory Two: Logic Gates}

\section{B1.1 Lab Procedure}

\section{B1.1.1 Part 1 - Problem Description}

As a Junior Engineer of 'ABC Printronics, you are provided a test unit that is used to control a Flexographic machine used to print product labels. This test unit contains 2 Switches, 1 Light outputs and a relay to familiarize yourself with the PLC I/Os and control problem specifications. Provide 2 different ways of creating a NAND Gate using this test unit provided.

Use 8 I/O PLC Module and make appropriate wirings for the power supply and ground. You are required to use a status bit for one of the methods alongside the address map provided below. 
Table B.1: Address Map for Problem in Part 1

\begin{tabular}{|c|l|l|}
\hline Address & \multicolumn{1}{|c|}{ Type } & Description/Functional Requirement \\
\hline I:0/2 & External Input & Switch \#1 \\
\hline I:0/4 & External Input & Switch \#2 \\
\hline B3:0/0 & Status Bit & To be used for the Relay \\
\hline O:0/2 & External Output & $\begin{array}{l}\text { Light\#1 which turns ON when the } \\
\text { NAND logic output is 1 }\end{array}$ \\
\hline
\end{tabular}

\section{B1.1.2 Part 2 - Problem Description}

The Engineering manager of 'ABC Printronics' has assigned you to yet another task to convert a digital logic wiring circuit of a packaging machine that is controlled by 5 Input Switches and a single output light. The light must only turn ON when the output to the logic circuit is ' 1 '.

Using these specifications and the digital logic circuit below, convert the circuit into a Boolean expression using the required laws of Boolean algebra. You are required to use a status bit for one of the methods alongside the address map provided below. Use 8 I/O PLC Module and make appropriate wirings for the power supply and ground.

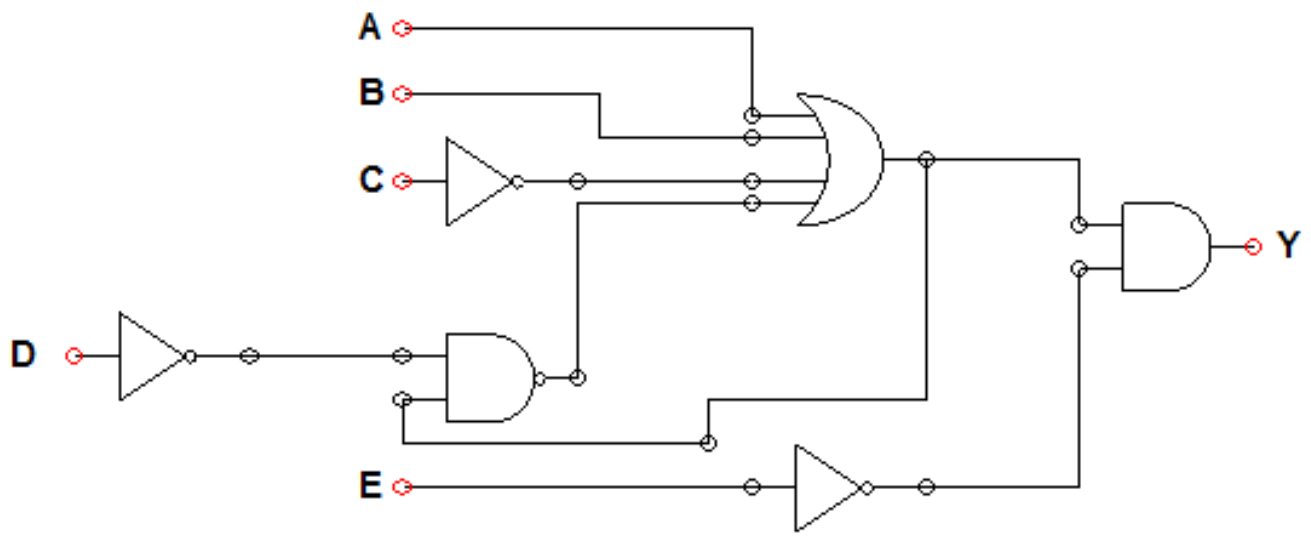

Figure B.1: Digital Logic Circuit for Problem Part 2 
Table B.2: Address Map for Problem in Part 2

\begin{tabular}{|c|c|c|}
\hline Address & Type & Description/Functional Requirement \\
\hline $\mathrm{I}: 0 / 2$ & External Input & Switch \#1 \\
\hline I:0/3 & External Input & Switch \#2 \\
\hline $\mathrm{I}: 0 / 4$ & External Input & Switch \#3 \\
\hline I:0/5 & External Input & Switch \#4 \\
\hline $\mathrm{I}: 0 / 6$ & External Input & Switch \#5 \\
\hline B3:0/0 & Internal & Internal Relay 1 \\
\hline B3:0/1 & Internal & Internal Relay 2 \\
\hline $\mathrm{O}: 0 / 1$ & $\begin{array}{l}\text { External } \\
\text { Output }\end{array}$ & Light which turns ON when the output to the Logic circuit is '1' \\
\hline
\end{tabular}

\section{B1.2 Laboratory Two - Solutions}
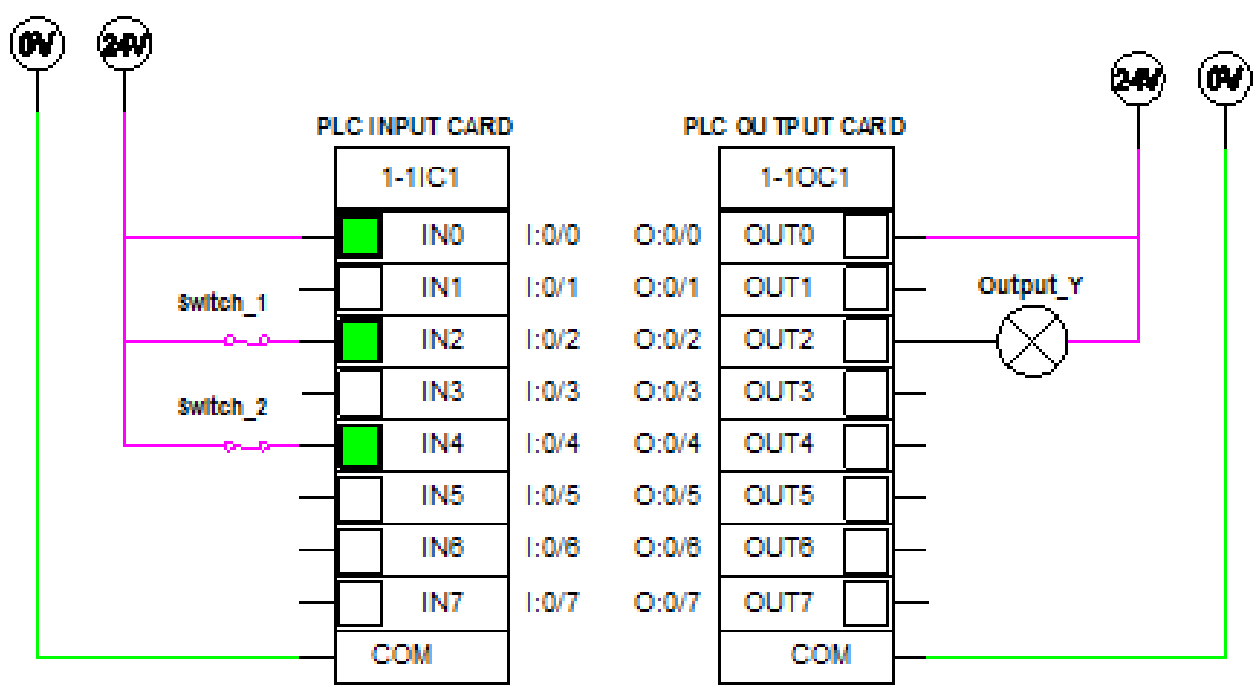

Figure B.2: I/O Wiring Diagram indicating Output Y in OFF state when the input switches are CLOSED. 

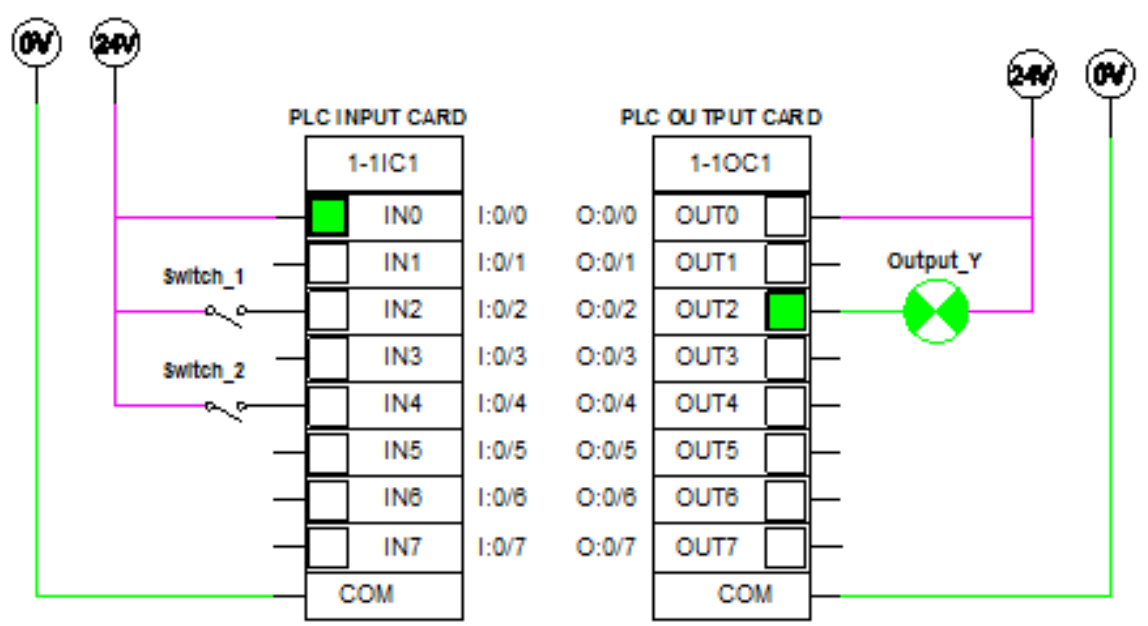

Figure B.3: I/O Wiring Diagram indicating Output $\mathrm{Y}$ in the ON state when the input switches are OPEN.

\section{B1.1.2 Part 1 Solutions}

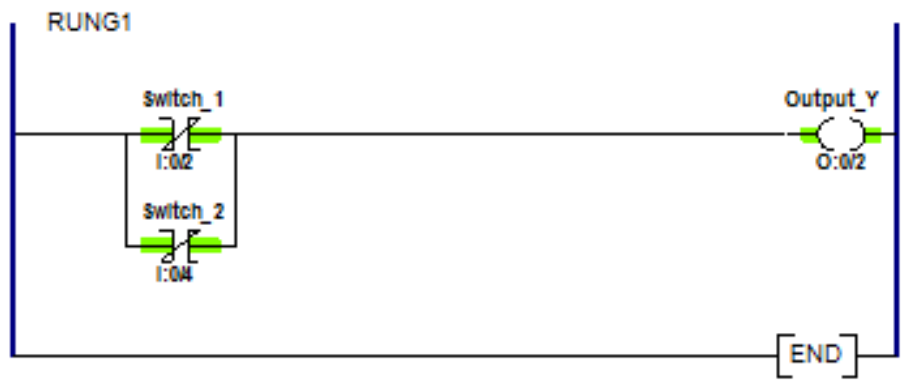

Figure B.4: Ladder Logic Program for Method 1

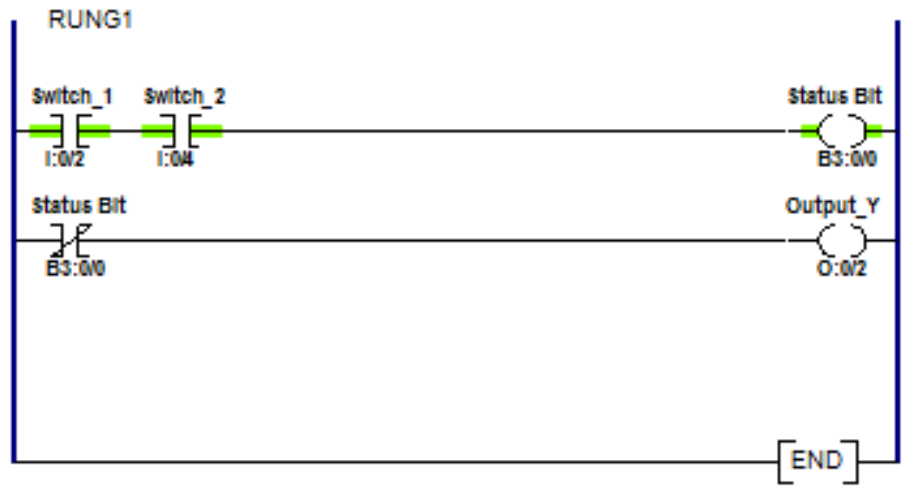

Figure B.5: Ladder Logic Program for Method 2 using the status bit internal variable 


\section{B1.1.2 Part 2 Solution}

Boolean Expression

$$
\begin{aligned}
Y & =E^{\prime} .\left\{\left(A+B+C^{\prime}\right)+\left[D^{\prime} .\left(A+B+C^{\prime}+D^{\prime}\right)\right]^{\prime}\right\} \\
& =E^{\prime} .\left\{\left(A+B+C^{\prime}\right)+\left[D+\left(A^{\prime} . B^{\prime} . C \text { C } D\right)\right]\right\} \text { (Using DeMorgan's Theorem) }
\end{aligned}
$$
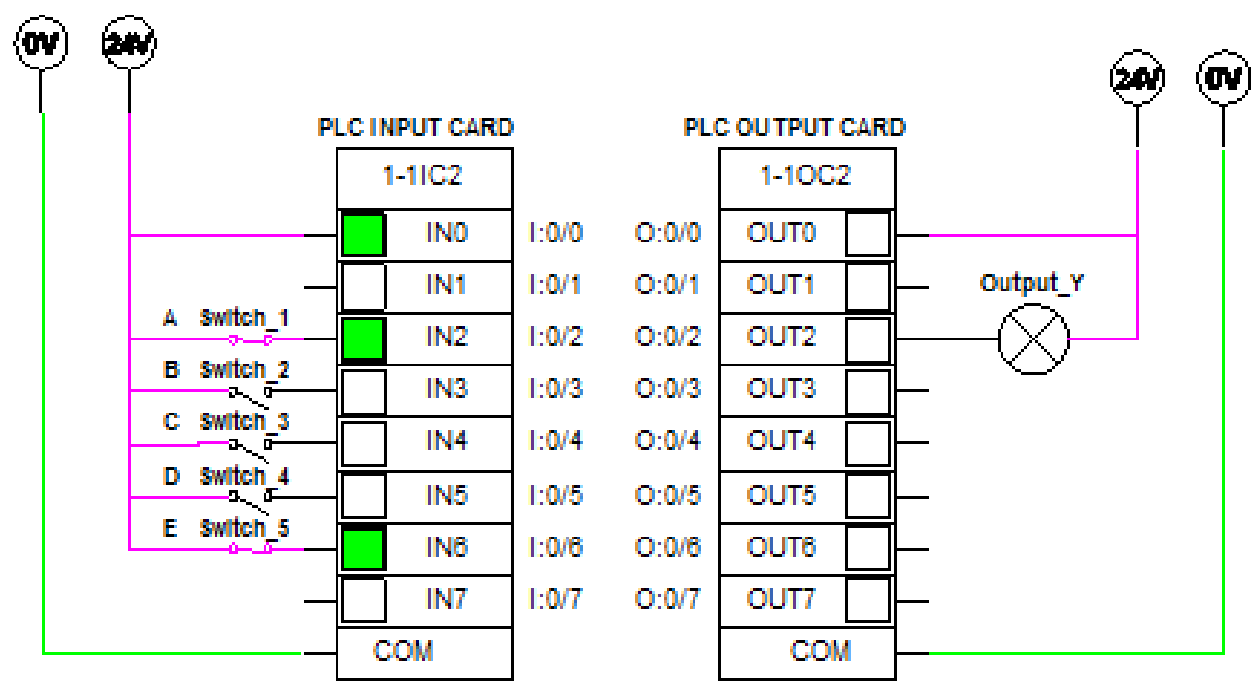

Figure B.6: Wiring Diagram on PLC Modules for Part 2 the Output $Y$ is in the OFF state 

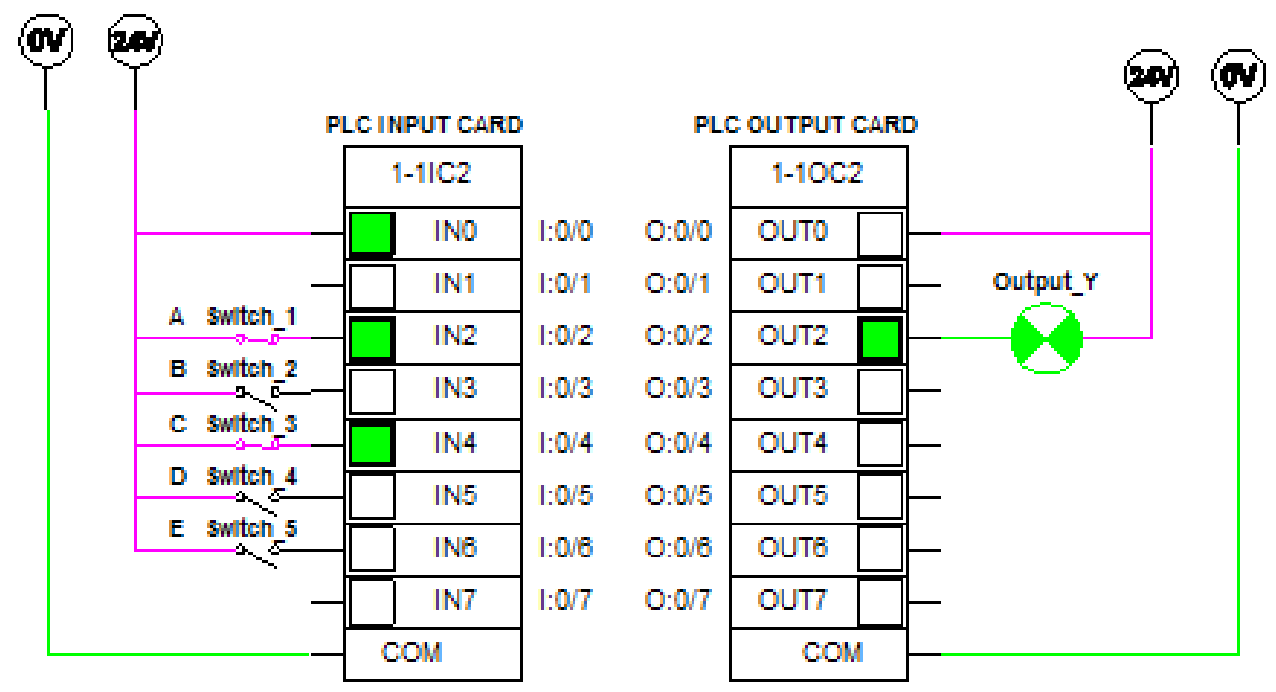

Figure B.7: Wiring Diagram on PLC Modules for Part 2 the Output Y is in the ON state

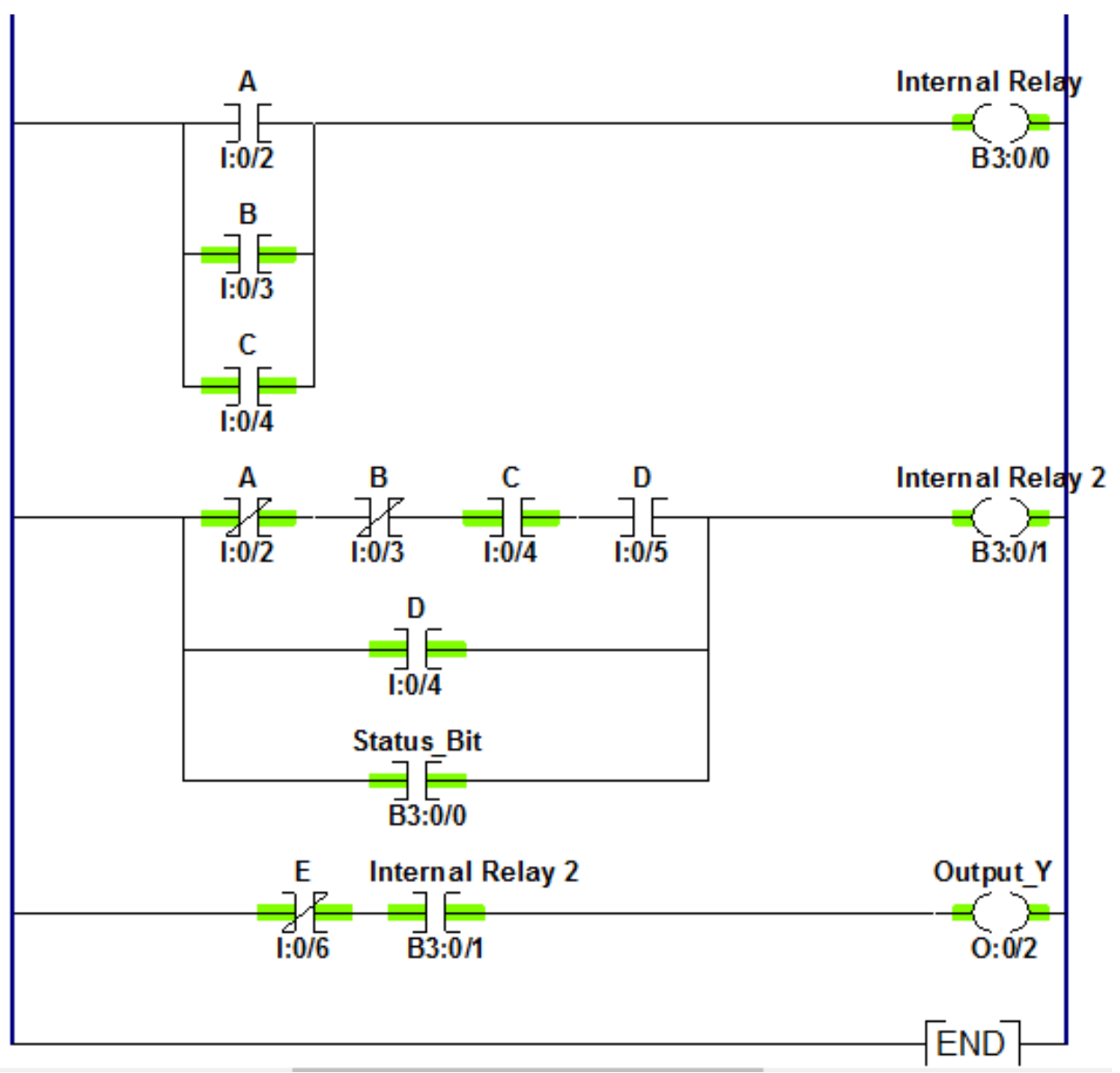

Figure B.8: Ladder Logic Program for Laboratory 2 


\section{Laboratory Three: Planer Machine Control}

\section{B2.1 Laboratory Procedure}

A Planar or Planer machine is a commonly used industrial tool that uses linear motion and a cutting tool to generate flat surfaces and repair injection moulds and stamping dies. Although milling machines have overtaken the planer machine in some industries, they are still widely used amongst smaller tool and die shops in the world today.

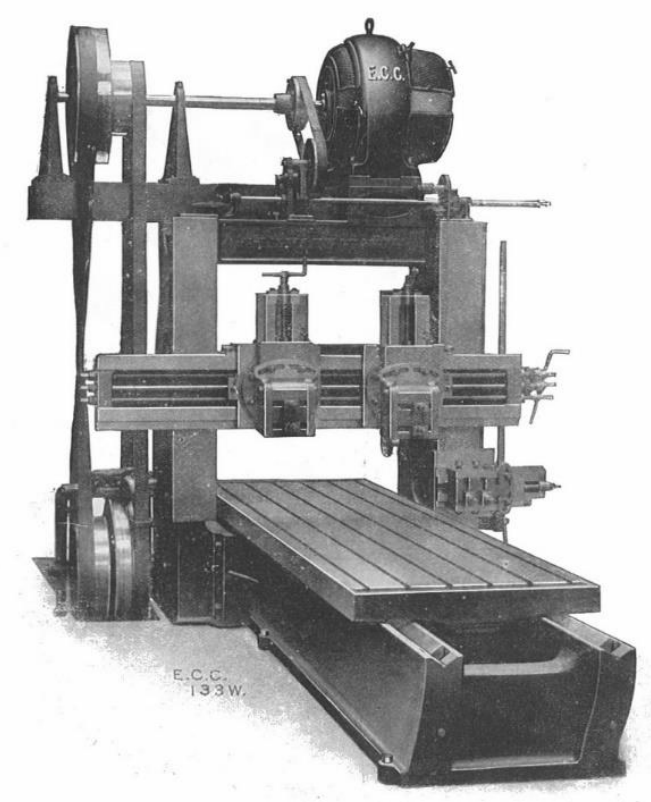

Figure B.9: Planer Machine [29]

The workpiece is typically placed on the table that moves back and forth in a linear motion. Thus, the cutting tool remains stationary on the machine whereas the table continues to move linearly controlled by switches 1LS and 2LS [27]. The control problem is bound by the following constraints:

i. Once the system is turned $\mathrm{ON}$ by pushing the start push-button, the motor begins to operate which is reversed upon job completion by the limit switches 1LS and 2LS.

ii. A provision of 'Jog' push-button must be allocated in the test-unit to operate the machine in incremental steps to calibrate the tool when necessary (This may not be required for the ladder logic but is to be allocated appropriately with an address). 
iii. Machine should not start if the machine table is lying in an extreme position.

iv. A delay period must be allotted to the motor prior to switching direction change from the left stroke to the right stroke.

v. In the event of overload tripping of the motor or if the stop push-button is pressed, the machine must instantly stop.

vi. The motor should not be able start unless the machine has been started (use the 'Interlocking' concept to interlock the motor pump as a pre-requisite for the operation of the machine.

You are required to appropriately determine the timers, contact coils and I/O connections to demonstrate the appropriate functioning of the logic and lab objectives. Use the Digital logic circuit to begin solving the control problem. In order to accommodate all the inputs, use a 16 point input/output module and drive it using $+24 \mathrm{~V}$ and ground supplies on Automation Studio.

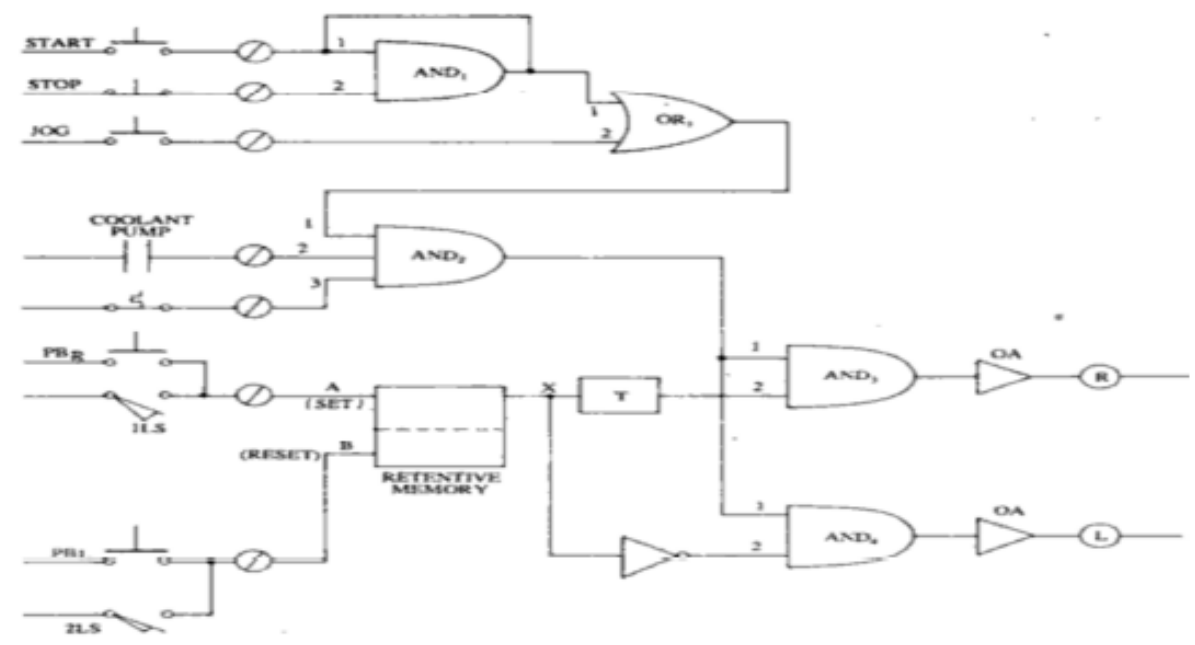

Figure B.10: Digital Logic Wiring Diagram for the Planer Machine Control System [25]

Table B.3: Address Map for Laboratory 3

\begin{tabular}{|c|l|l|}
\hline Address & \multicolumn{1}{|c|}{ Type } & \multicolumn{1}{c|}{ Description/Functional Requirement } \\
\hline I:0/1 & External Input & Normally-Open (NO) Start Pushbutton \\
\hline I:0/2 & External Input & Normally-Closed (NC) Stop Pushbutton \\
\hline I:0/3 & External Input & Normally-Open Jog Pushbutton \\
\hline I:0/4 & External Input & Toggle Switch NO - Pump \\
\hline
\end{tabular}




\begin{tabular}{|c|l|l|}
\hline I:0/5 & External Input & Toggle Switch NC - Overload \\
\hline I:0/6 & External Input & Toggle Switch NO - 1LS \\
\hline I:0/7 & External Input & Normally-Open (NO) Set Pushbutton \\
\hline I:0/8 & External Input & Normally-Open (NO) Set Pushbutton \\
\hline I:0/9 & External Input & Toggle Switch NO - 2LS \\
\hline O:0/1 & External Output & Indicator Light - L1 Output \\
\hline O:0/2 & External Output & Indicator Light - MR' Output \\
\hline O:0/3 & External Output & Indicator Light - MR (right stroke) Output \\
\hline O:0/4 & External Output & Indicator Light - ML (left stroke) Output \\
\hline
\end{tabular}

\section{B2.2 Laboratory Three - Solution}
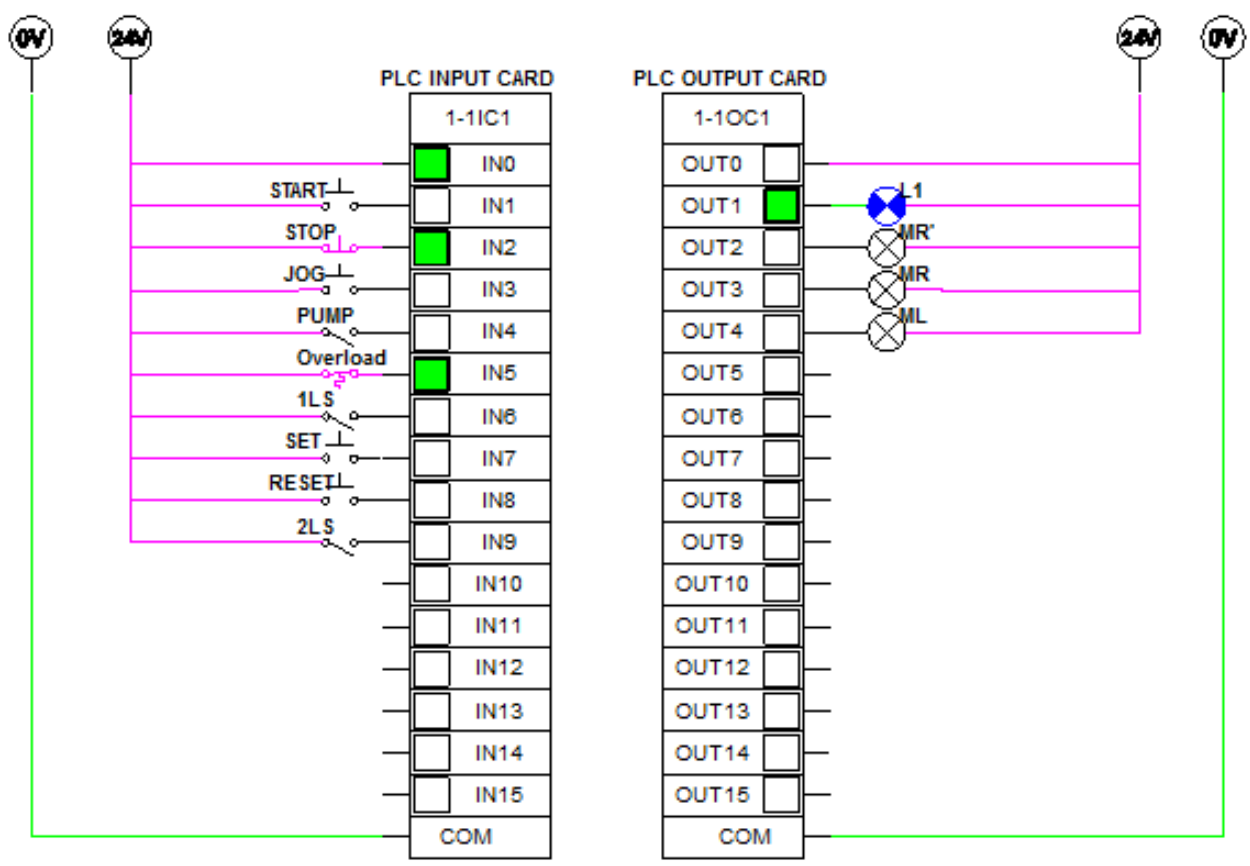

Figure B.11: PLC I/O Wiring Diagram for the Planer Machine Control System 


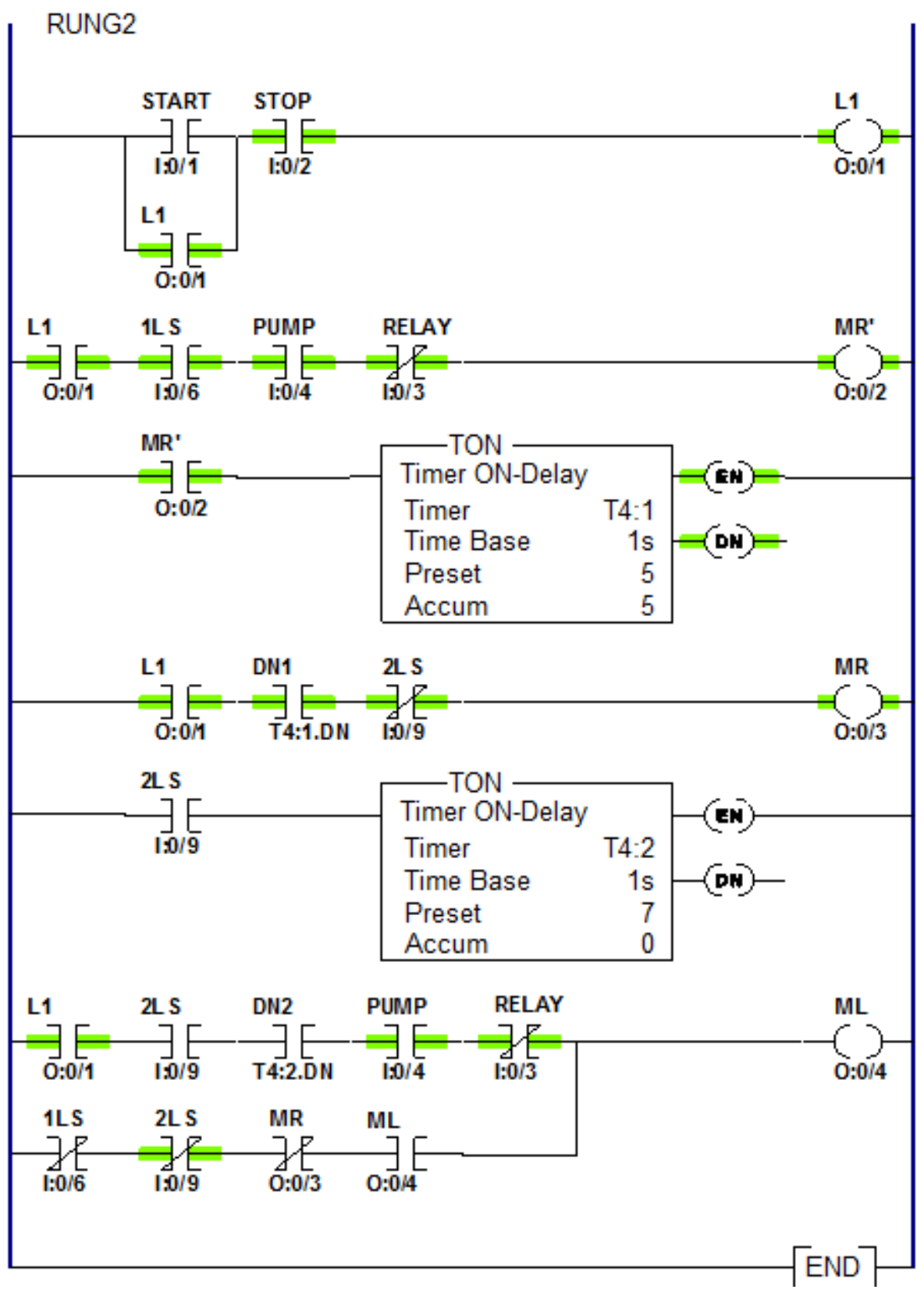

Figure B.12: Ladder Logic Program for the Planer Machine Control System 


\section{Appendix C: Applications in the Oil \& Chemical Industry}

PLCs have found a significant role the oil and chemical industries. They are also used in pharmaceutical firms and the food industry to perform numerous operations. Common applications in these industries include Batch operations for material handling, vial washing and mixing operations as well as in pump control systems.

In the following labs, students gain an understanding of how these batch operations are performed as well as how PLCs are efficiently used to perform the 1 in these industries.

\section{Laboratory Four: Batch Mixing}

\section{C1.1 Laboratory Procedure}

In Oil and chemical applications, vial mixing and washing is an important operation that needs to be carried out in a measured and systematic way. Two or more liquids are typically mixed with one another to form a batch wherein the level of the liquid in the tank is detected using sensors. The rate of liquid flow is fixed and the time and proportion is controlled using limit switches.

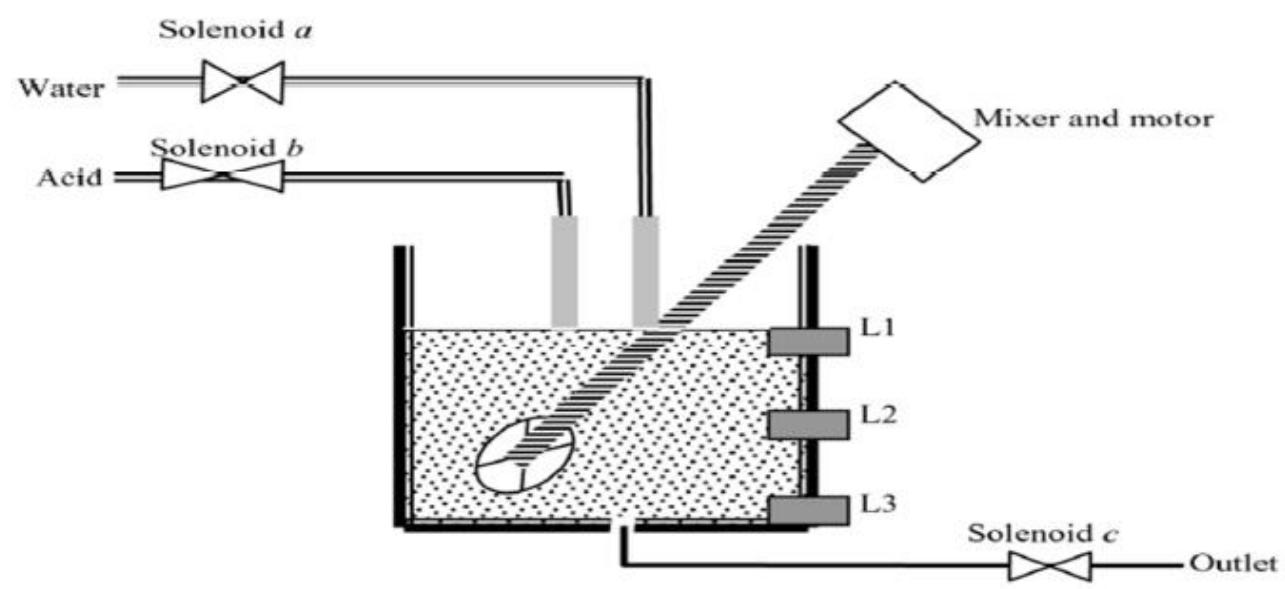

Figure C.1: Lab 4 - Batch Mixing System Diagram [25] 
In this application, you are required to dilute a chemical by mixing it with water in a ratio of 3:2. Three level sensors L1, L2 and L3 detect the level of liquid in the tank. Solenoid valves a,b and c control the inlet and outlet of liquid to and from the tank respectively. Once the ratio has been attained in the tank, the motor mixer begins to operate to blend the 2 liquids.

You are required to begin operation with the assumption that all the valves are initially closed, the tank is empty and the system is non-operational. A Reset pushbutton must be included to reset the timer once it has timed out.

Use a timer with a preset value of 10 along with contact coils and I/O connections to demonstrate the appropriate functioning of the logic and lab objectives. Use an 8 point input/output PLC module and $+24 \mathrm{~V}$ and ground supplies on Automation Studio to drive the PLC.

Table C.1: Address Map for Laboratory 4

\begin{tabular}{|c|l|l|}
\hline Address & \multicolumn{1}{|c|}{ Type } & \multicolumn{1}{c|}{ Description/Functional Requirement } \\
\hline I:0/1 & External Input & Normally-Open (NO) Start Pushbutton \\
\hline I:0/2 & External Input & Toggle Switch NO - L3 Valve \\
\hline I:0/3 & External Input & Toggle Switch NO - L2 Valve \\
\hline I:0/4 & External Input & Toggle Switch NO - L1 Valve \\
\hline I:0/5 & External Input & Normally-Open (NO) Reset Pushbutton \\
\hline O:0/1 & External Output & Indicator Light - System ON \\
\hline O:0/2 & External Output & Indicator Light - Solenoid C ON \\
\hline O:0/3 & External Output & Indicator Light - Solenoid A ON \\
\hline O:0/4 & External Output & Indicator Light - Solenoid B ON \\
\hline O:0/5 & External Output & Indicator Light - Mixer Motor Status \\
\hline
\end{tabular}




\section{C1.2 Laboratory Four - Solution}
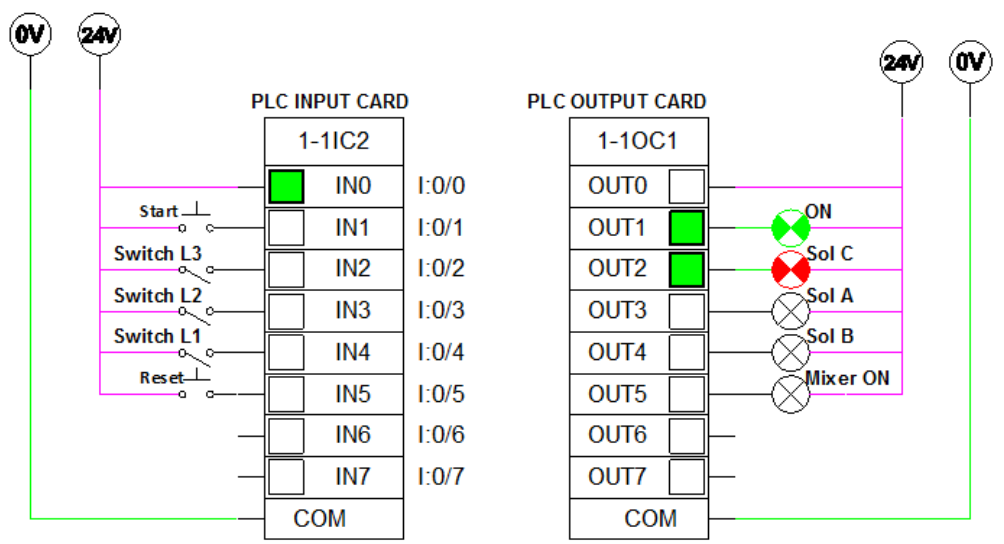

Figure C.2: I/O Wiring Diagram for the Batch Mixing system

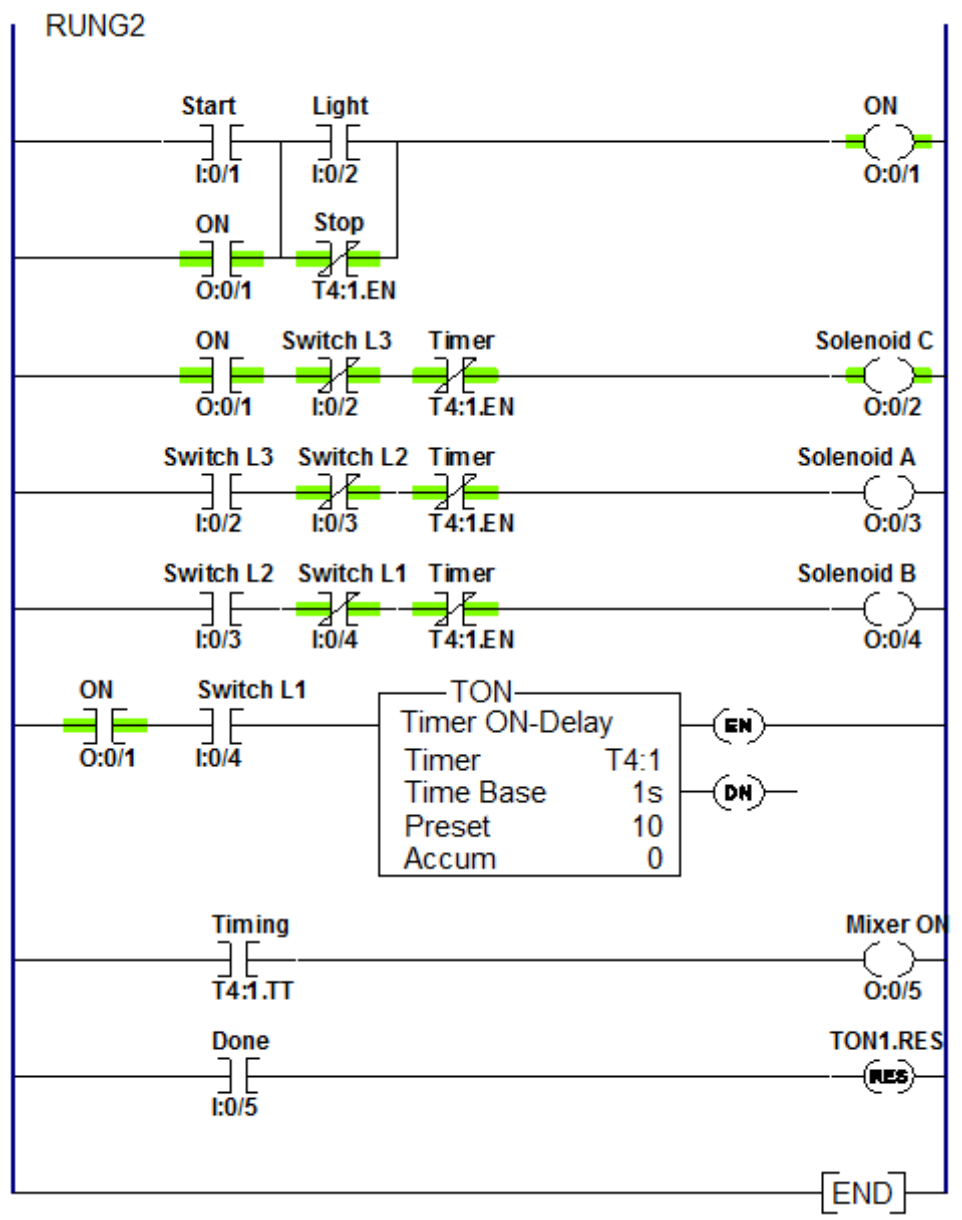

Figure C.3: Ladder Logic program for the Batch Mixing system 


\section{Laboratory Five: Bottle Filling System}

\section{C2.1 Laboratory Procedure}

In mass chemical or liquid packaging operations, PLCs are often used to operate the machines or conveyor belts to detect the position of the bottles or packages following which the filling operation begins. Once the bottle has been filled, the PLC appropriately moves it along the conveyor for further sealing and labelling operations. If a manual workforce was employed to perform individual filling and packaging operations, the process can become very time consuming and tedious. This is why PLCs are used by which the process is significantly simplified and can be altered with programming to perform the desired operation.

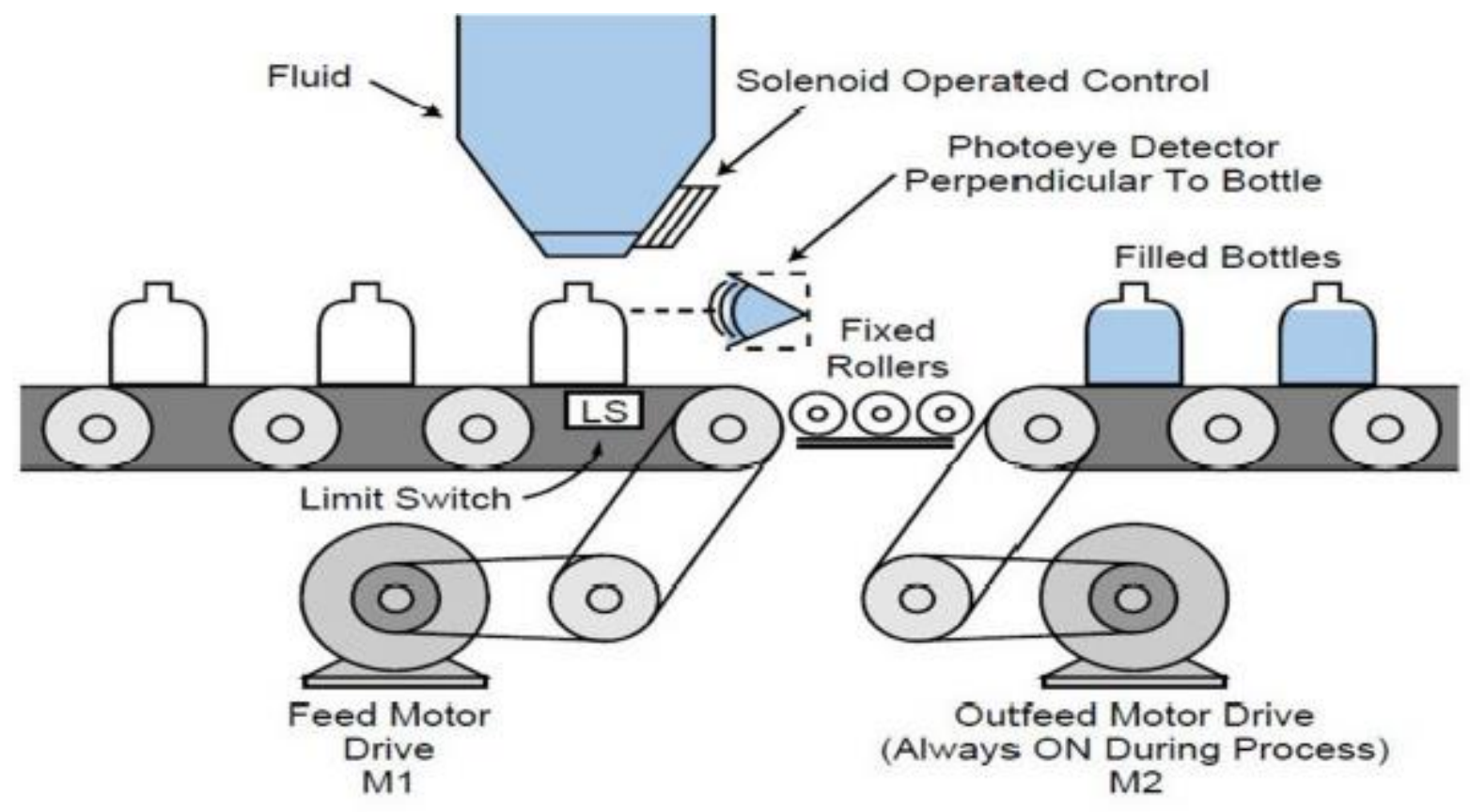

Figure C.4: Bottle Filling System for Laboratory 5 [25]

Although the position of the bottle is typically detected by a position sensor on the system, we indicate the detection of a bottle in our control problem using a limit switch (LS) following which a timer begins for a wait of 10s. Again, another switch is to be used as a photodetector to determine when the bottle has been filled to the required level. Completion of the bottle fill condition is indicated with an output indicator light which begins the fixed rollers and the motor 
M2 to move the filled bottles onto the next operation. However, as long as the limit switch (LS) has not been signalled, the M1 motor continues to operate. This motor M1 is to drive the belt to appropriately position the bottle below the solenoid water nozzle.

Program a PLC using ladder logic programming to perform this bottle filling system operation as described. Use appropriate timers and contact coils to demonstrate the appropriate functioning of the control system operation and lab objectives. Use an 8 point input/output PLC module and $+24 \mathrm{~V}$ and ground supplies on Automation Studio to drive the PLC.

Table C.2: Address Map for Laboratory 5

\begin{tabular}{|c|l|l|}
\hline Address & \multicolumn{1}{|c|}{ Type } & \multicolumn{1}{|c|}{ Description/Functional Requirement } \\
\hline I:0/1 & External Input & Normally-Open (NO) Start Pushbutton \\
\hline I:0/2 & External Input & Normally-Open (NO) Stop Pushbutton \\
\hline I:0/3 & External Input & Toggle Switch NO - Limit Switch (LS) \\
\hline I:0/4 & External Input & Toggle Switch NO - Photodetector \\
\hline I:0/5 & External Input & Toggle Switch NO - Filled Bottle Status \\
\hline O:0/1 & External Output & Indicator Light - Motor M1 Status \\
\hline O:0/2 & External Output & Indicator Light - Motor M2 Status \\
\hline O:0/3 & External Output & Solenoid - Water Filler Nozzle System \\
\hline O:0/4 & External Output & Flash Light \\
\hline O:0/5 & External Output & Indicator Light - Filled Bottle Condition \\
\hline
\end{tabular}




\section{C2.2 Laboratory Five - Solution}

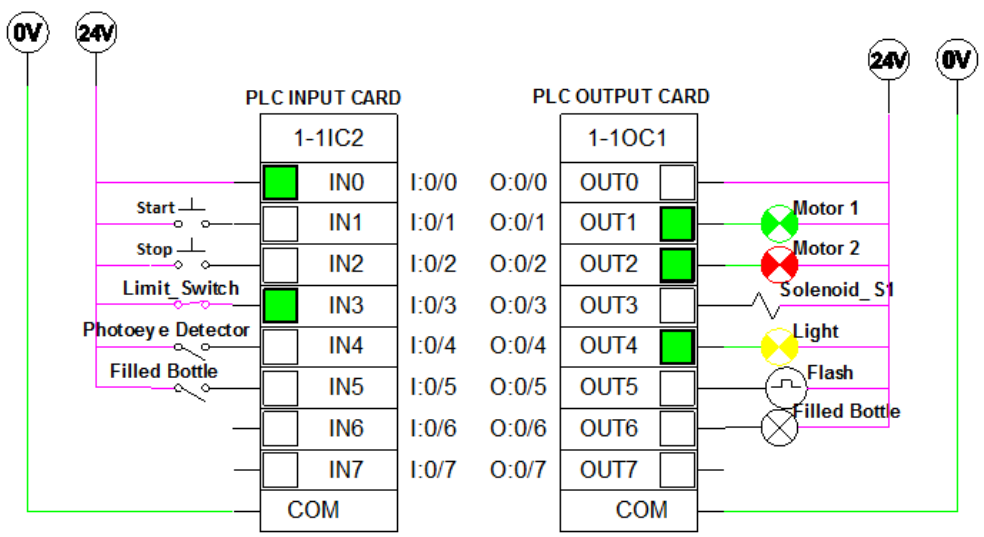

Figure C.5: PLC I/O Wiring Diagram for Laboratory 5

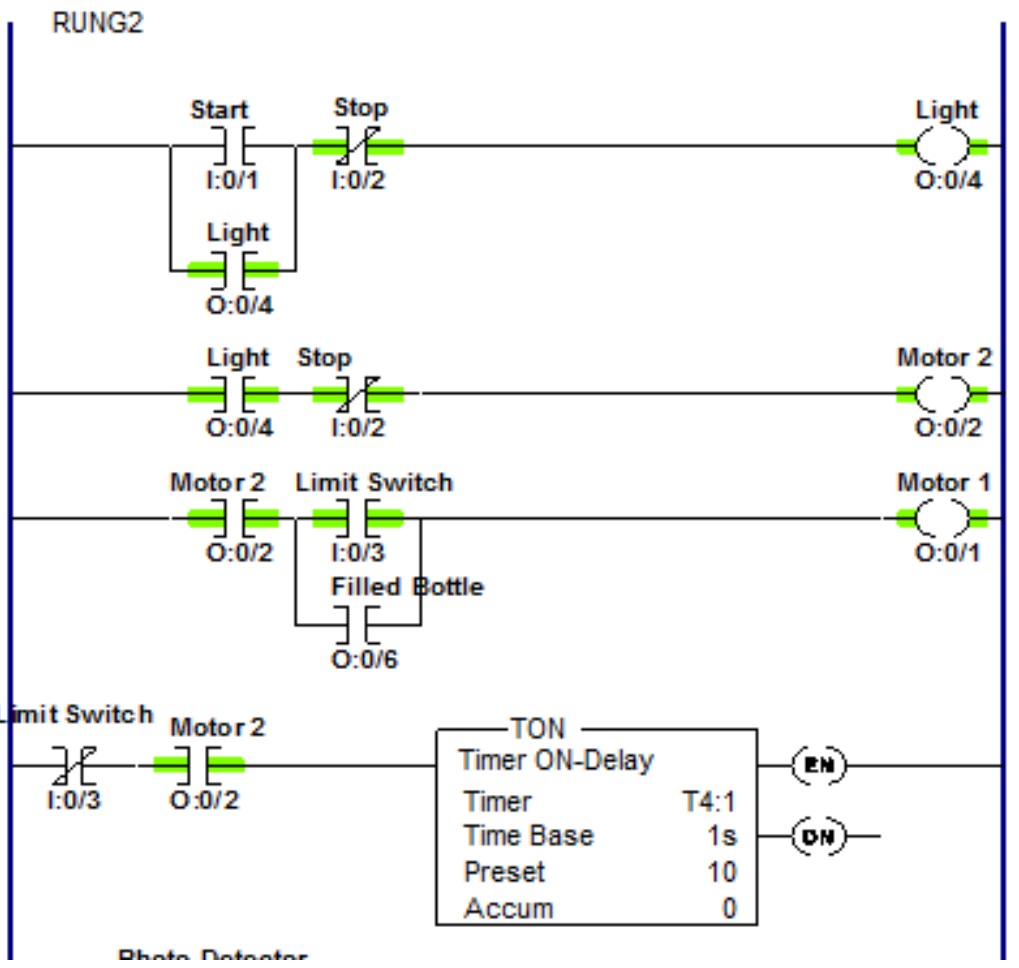

Figure C.6: Part 1 of the Ladder Logic program for Laboratory 5

Figure C.6: Part 1 of the Ladder Logic program for Laboratory 5 


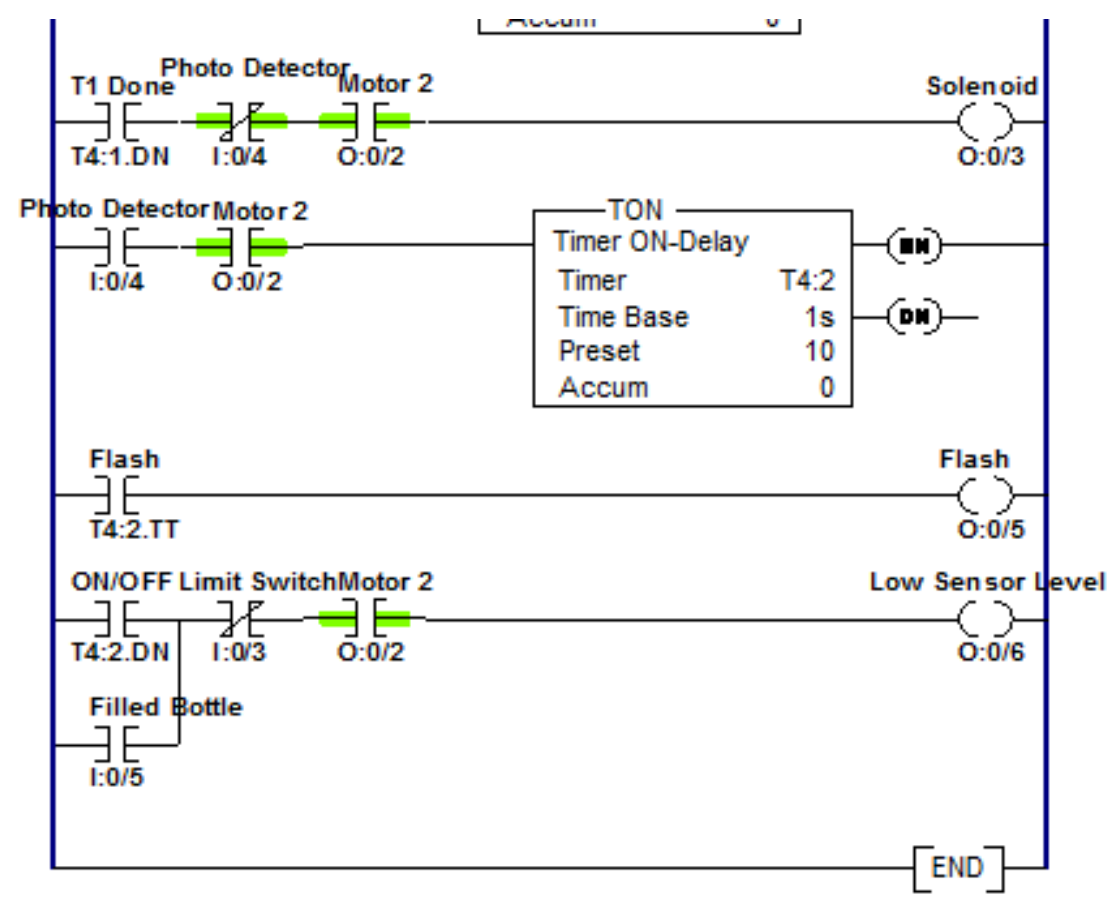

Figure C.7: Part 2 of the Ladder Logic program for Laboratory 5

\section{Appendix D: Applications in the Automotive \& Aerospace Industry}

In competitive industries such as Automobile and Aerospace, one of the fastest growing and increasingly demonstrated technologies is the use of PLCs. Applications of their use can be found in the manufacture of products, material handling, monitoring and data logging as well as fault detection. For such applications, PLCs are required to control multiple output devices simultaneously to perform an operation. Using PLCs to control these outputs in the desired manner and change their operation when required is what makes PLCs highly suitable for these applications.

Furthermore, timing and comparison constraints also play a crucial role in the successful operation of a task. This can be seen at Traffic light junctions where each light needs to be timed for a certain amount of time or in a manufacturing plant where machines need to alternate in 
operation to produce batches. This lab is designed to introduce students to handle these type of constraints and appropriately determine which ladder logic functions to use to solve the control problems presented below in 2 parts.

In the following labs, students will learn to program the PLCs to solve 3 such control problems. Applications of these problems are intertwined with other industries or a variation of these control problems are found in the industry.

\section{Laboratory Six: Air Conditioning System}

\section{D1.1 Laboratory Procedure}

Control problems pertaining to air-conditioning systems is not specific to only Automobile and Aerospace industries but is a widespan technological problem that can be found almost everywhere. One such Air-conditioner is the 3 stage A/C which consists of motor compressors to operate the $\mathrm{A} / \mathrm{C}$ and a thermostat to control and maintain the temperature at the desired level. The control logic for the A/C can be seen in the figure below.

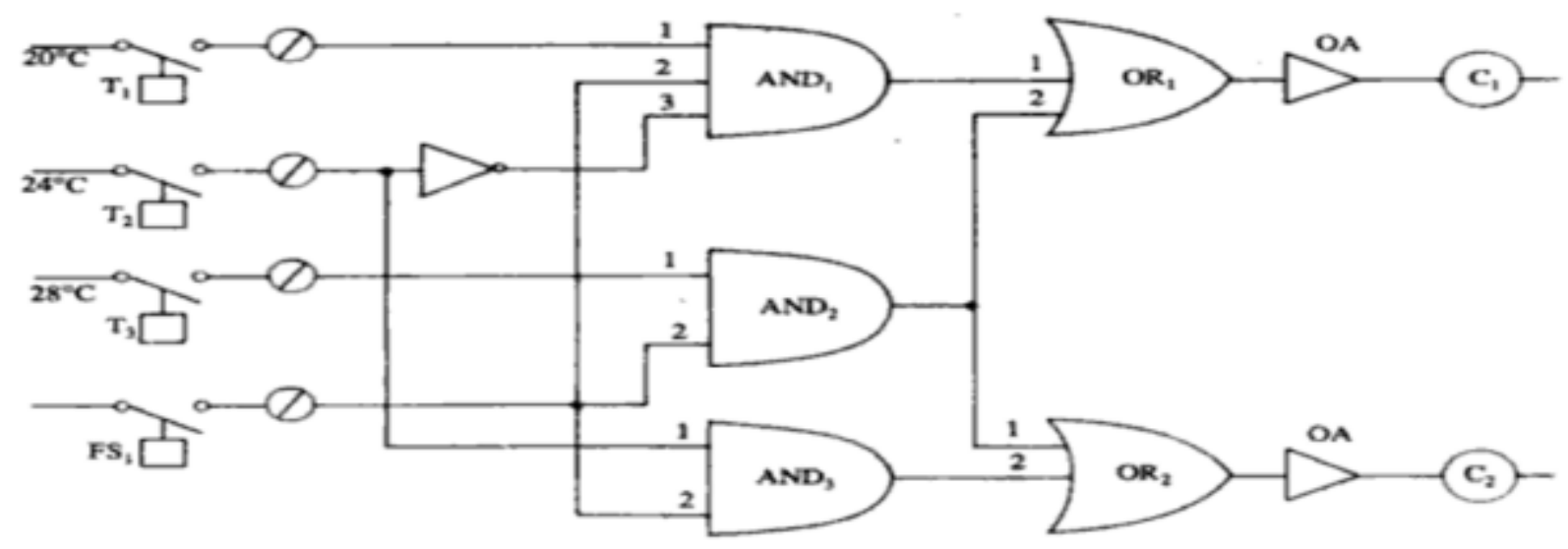

Figure D.1: 3 Stage Air-Conditioner System for Laboratory 6 [25]

The outputs of the digital logic circuit are $\mathrm{C} 1$ and $\mathrm{C} 2$ which are designators for the 2 compressor motors in the A/C system. The following constraints determine the system:

i) Three thermostats must be present in the system which shall be substituted by toggle switches. The thermostats can be turned $\mathrm{ON}$ and $\mathrm{OFF}$ to visualize the various 
operational conditions. The temperature settings present in the circuit diagram can be ignored here.

ii) In order for either of the compressors $\mathrm{C} 1$ or $\mathrm{C} 2$ to be operational, the Flow Switch (FS1) must be closed.

iii) Terminal 2 of all the gates are driven by the status of FS1. Thus, when FS1 is closed, a high logic signal is sent to the Terminal 2 of all the logic AND gates.

Note: Operation Amplifiers (OA) between the compressors and OR gate outputs can be ignored for this ladder logic operation.

Simulate the Air Conditioning system using ladder logic programming and the digital logic circuit to demonstrate the appropriate functioning of the control system operation. Use an 8 point input/output PLC module and $+24 \mathrm{~V}$ and ground supplies on Automation Studio to drive the PLC.

Table D.1: Address Map for Laboratory 6

\begin{tabular}{|c|l|l|}
\hline Address & \multicolumn{1}{|c|}{ Type } & \multicolumn{1}{c|}{ Description/Functional Requirement } \\
\hline I:0/1 & External Input & Toggle Switch NO - Thermostat \#1 \\
\hline I:0/2 & External Input & Toggle Switch NO - Thermostat \#2 \\
\hline I:0/3 & External Input & Toggle Switch NO - Thermostat \#3 \\
\hline I:0/4 & External Input & Toggle Switch NO - Flow Switch (FS1) \\
\hline O:0/1 & External Output & Indicator Light - Output AND1 \\
\hline O:0/2 & External Output & Indicator Light - Output AND2 \\
\hline O:0/3 & External Output & Indicator Light - Output AND3 \\
\hline O:0/4 & External Output & Indicator Light - Contact Compressor 1 \\
\hline O:0/5 & External Output & Indicator Light - Contact Compressor 2 \\
\hline
\end{tabular}


D1.2 Laboratory Six - Solution
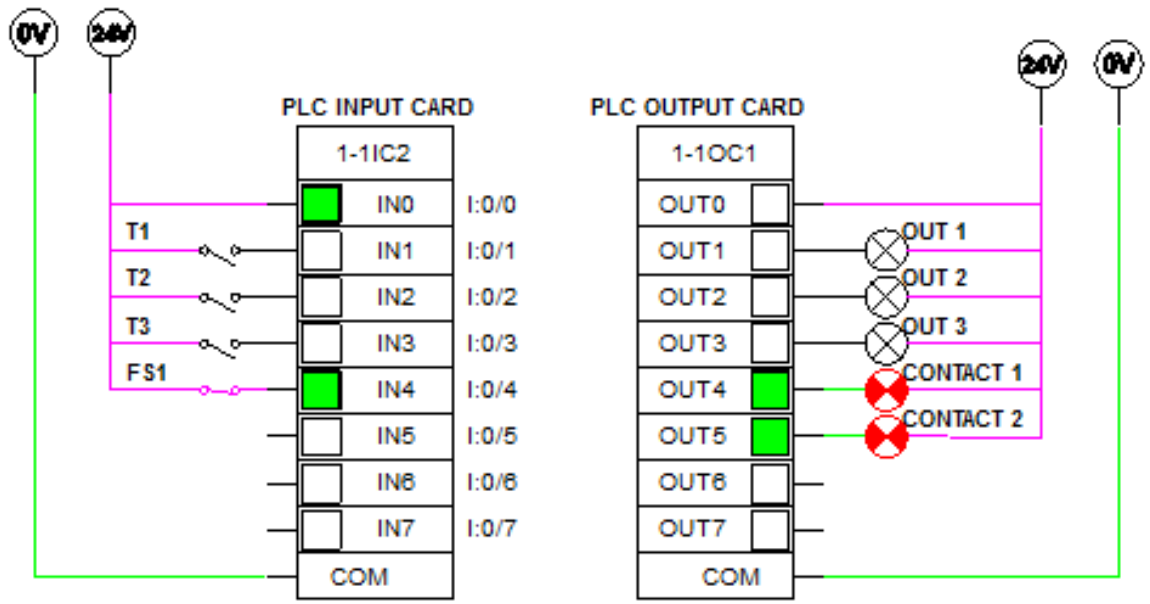

Figure D.2: PLC I/O Wiring Diagram for Laboratory 6

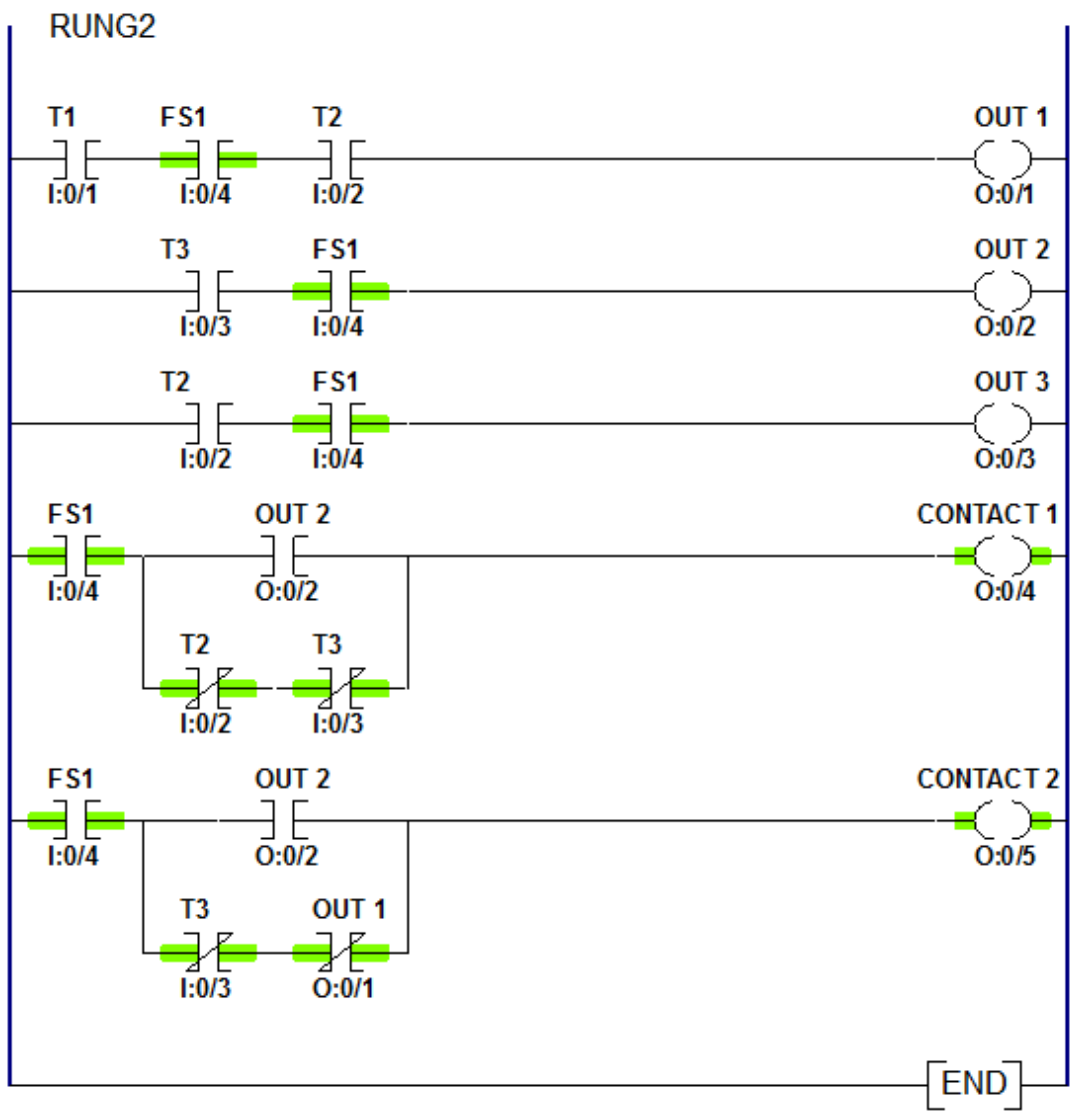

Figure D.3: Ladder Logic program for Laboratory 6 


\section{Laboratory Seven: Water Tank Monitoring}

\section{D.2.1 Laboratory Procedure}

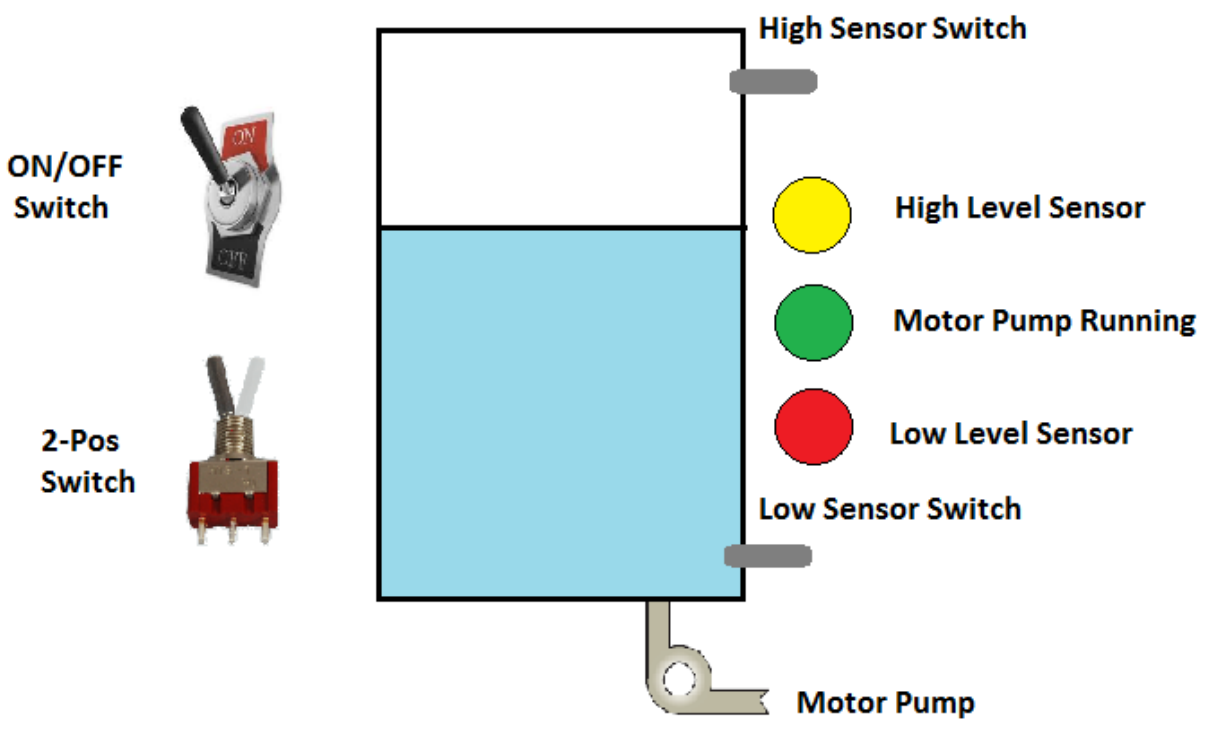

Figure D.4: Illustration of the Water Tank Monitoring System

You have been contracted by 'YYZ Hydro' to use PLC ladder logic to control a water tank level system for a Chemical Manufacturing company. The water tank is to be filled and emptied as desired constantly over a 24 -hour period. The chemical manufacturing company has provided YYZ Hydro with the following constraints and specifications [5]:

i) The water tank system operates in 2 modes - Manual Mode \& Automatic Mode.

a. In the Manual mode - the water pump motor can be turned ON to fill the tank with water as long as the water level in the tank is not low.

b. In the Automatic Mode, the pump begins to operate when the water level is at a HIGH level and continues to operate until the LOW level sensor is actuated. Once the LOW level sensor is activated, the pump stops.

ii) To test the appropriate operation, the manufacturing company has suggested to use 2 sensor switches in place of level sensors to indicate the 'High' and 'Low' water level in the tank. 
iii) The status of the water pump must be indicated with 3 outputs lights (Red Water Level High, Yellow - Level Low \& Green - Motor Pump is in operation).

iv) Interlocking

a. The tank is controlled by a single ON/OFF switch and will not operate under any circumstances if the switch is OFF.

b. Since we know the water level cannot be HIGH and LOW at the same time, an interlock needs to be incorporated such that both sensors are not activated at the same time (in this case - the two status LEDs for the sensors cannot be $\mathrm{ON}$ at the same time).

v) Use the Latch/Unlatch mechanism to latch onto the Motor Pump based on when the above conditions are TRUE or FALSE.

Using these specifications and the address map below, provide a solution for the control problem using the Latch/Unlatch method. Use 8 I/O PLC Module and make appropriate wirings for the power supply and ground.

Table D.2: Address Map for Lab 3 Control Problem

\begin{tabular}{|c|c|c|}
\hline Address & Type & Description/Functional Requirement \\
\hline $\mathrm{I}: 0 / 2$ & External Input & Pushbutton \#1 \\
\hline $\mathrm{I}: 0 / 3$ & External Input & 2 Position Switch (Manual Mode \& Auto \\
\hline $\mathrm{I}: 0 / 4$ & External Input & Mode) \\
\hline $\mathrm{I}: 0 / 5$ & External Input & Low Sensor Switch \\
\hline $\mathrm{I}: 0 / 6$ & External Input & High Sensor Switch \\
\hline $\mathrm{O}: 0 / 1$ & $\begin{array}{l}\text { External } \\
\text { Output }\end{array}$ & Motor \\
\hline $\mathrm{O}: 0 / 4$ & $\begin{array}{l}\text { External } \\
\text { Output }\end{array}$ & $\begin{array}{l}\text { Green LED - to indicate the when the Motor } \\
\text { Pump is RUNNING. }\end{array}$ \\
\hline $\mathrm{O}: 0 / 5$ & $\begin{array}{l}\text { External } \\
\text { Output }\end{array}$ & Red LED - to indicate Low Water Level \\
\hline $0: 0 / 6$ & $\begin{array}{l}\text { External } \\
\text { Output }\end{array}$ & Yellow LED - to indicate High Water Level \\
\hline
\end{tabular}




\section{D.2.2 Laboratory Seven - Solutions}
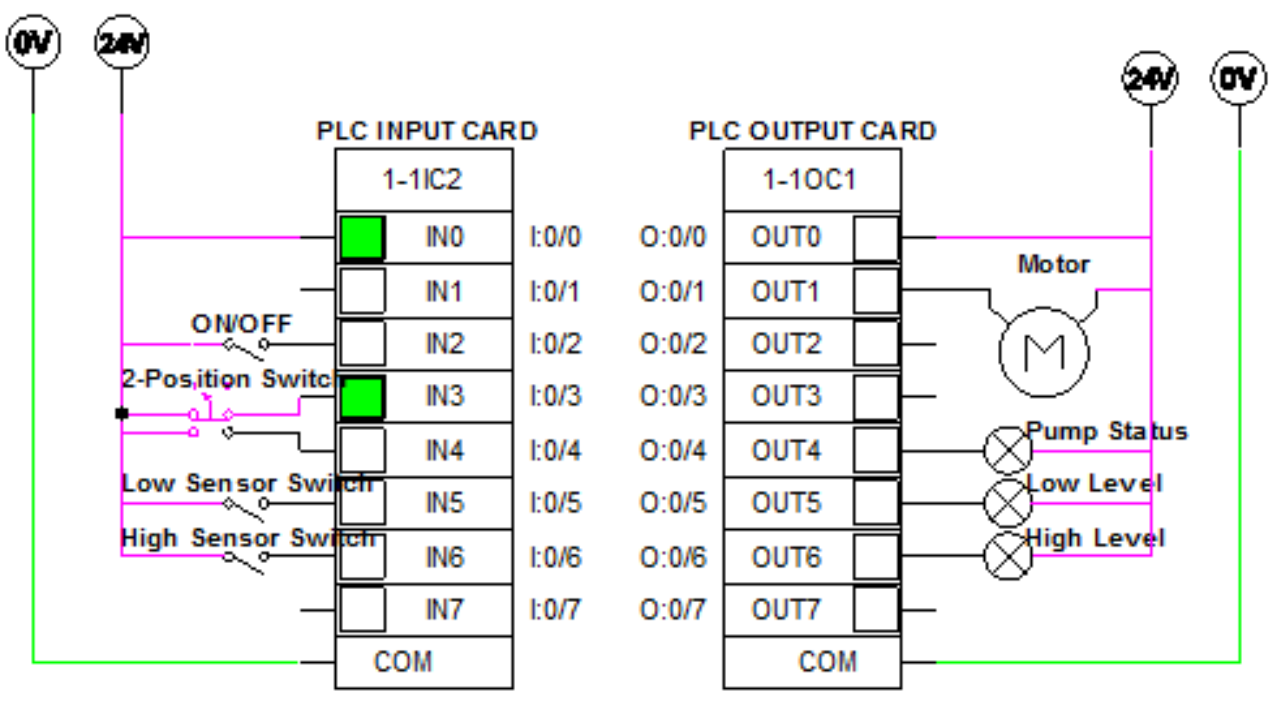

Figure D.5: I/O Wiring Diagram on the PLC Modules for Lab 3 when the system is in OFF state and the Motor Pump is non-functional
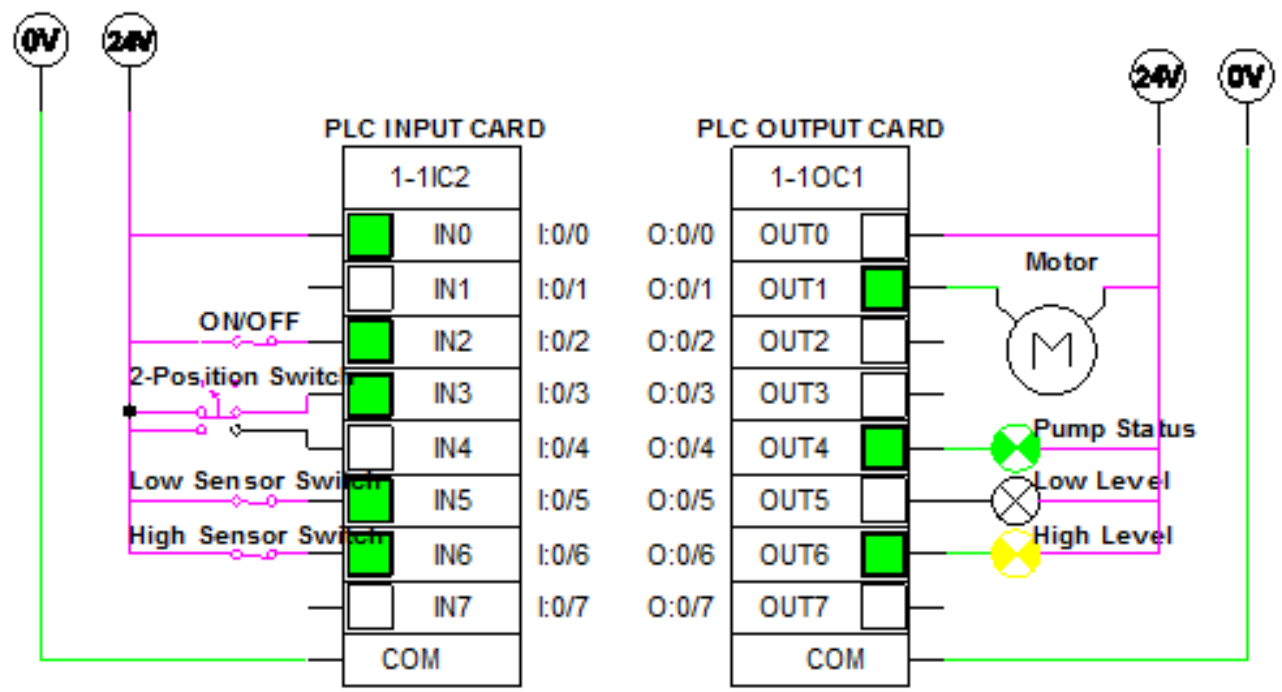

Figure D.6: I/O Wiring Diagram on the PLC Modules for Lab 3 when the water level is HIGH and the Motor Pump has begun operation to lower the water level in the tank. The tank is currently operating in Manual Mode. 

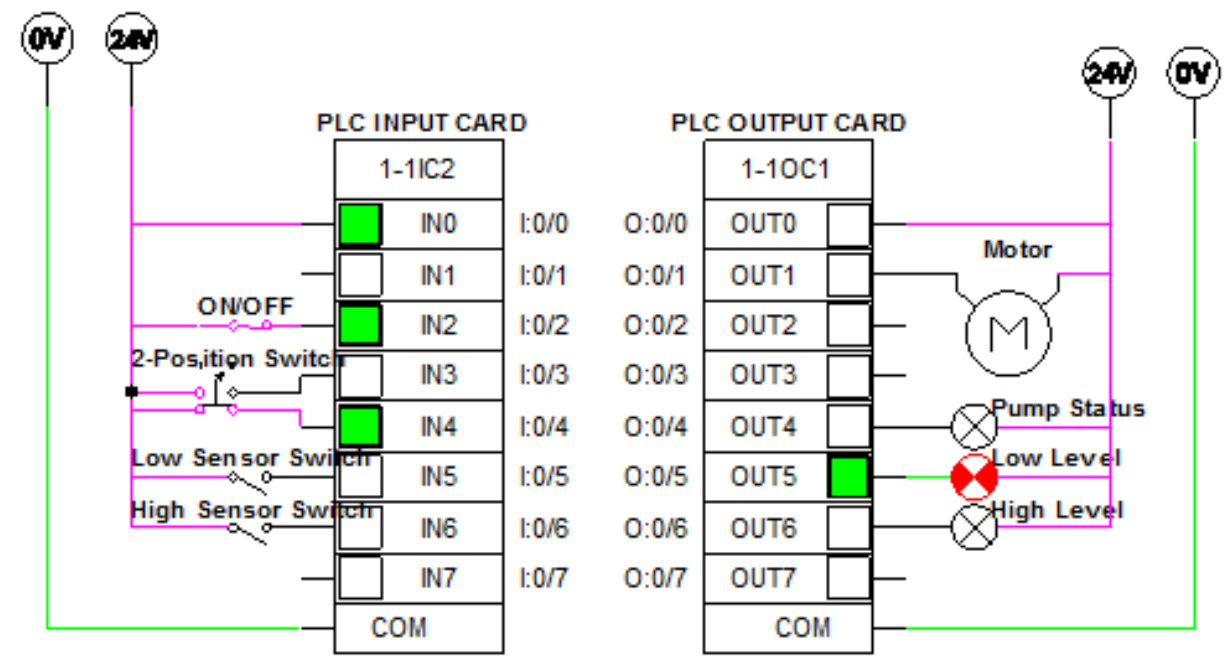

Figure D.7: I/O Wiring Diagram on the PLC Modules for Lab 3 when the water level is LOW and the Motor Pump has stopped. The tank is currently operating in Auto Mode.

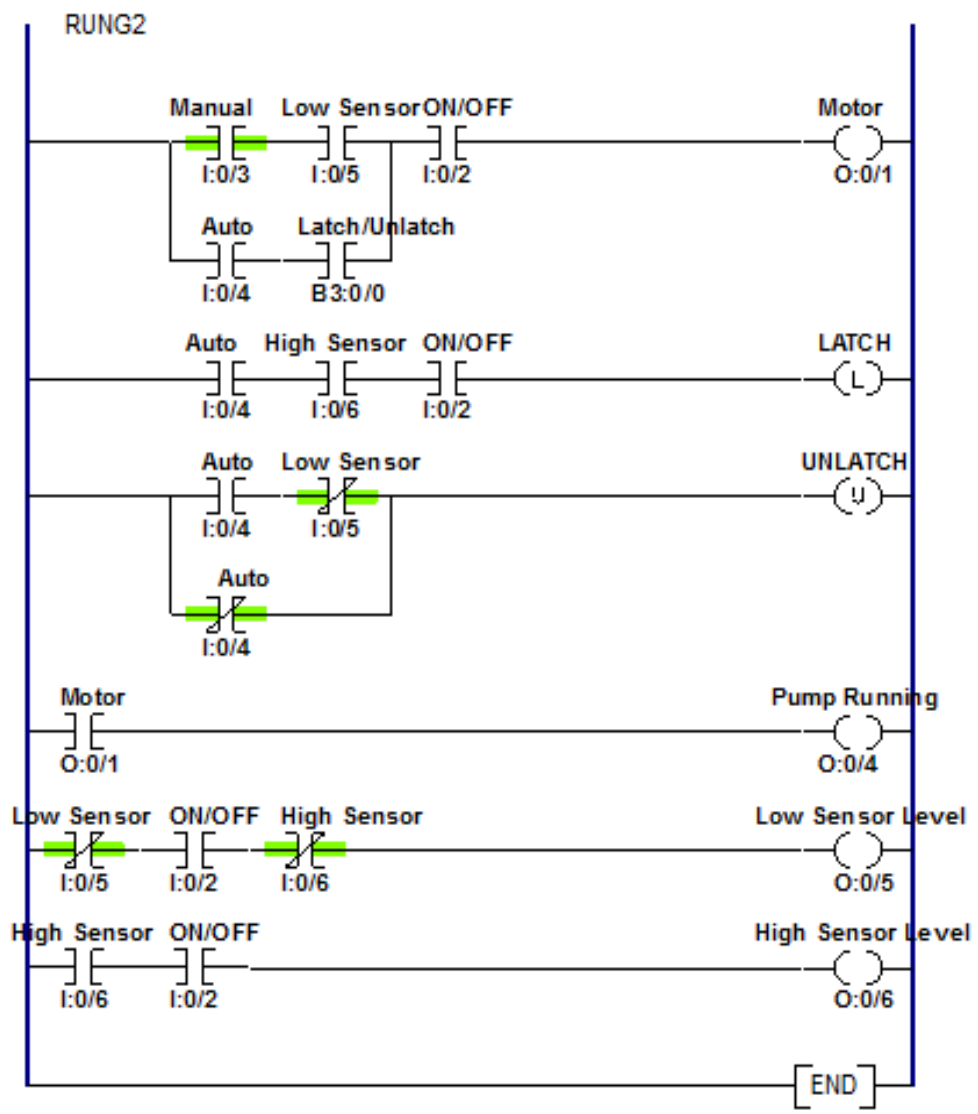

Figure D.8: Ladder Logic Program for Lab 3 


\section{Laboratory Eight: Constraint Handling}

\section{D.3.1 Laboratory Procedure}

\section{D.3.1.1 Part 1 - Problem Description}

Use a TON timer to turn on a cooling fan for 10 seconds using a Push Button. Wire an 8 Input/8 Output PLC module in Automation Studio with a switch to start \& stop the fan and 2 output LEDs to display the status of the fan i.e. whether it is ON or OFF. Use a $+24 \mathrm{~V}$ supply and $0 \mathrm{~V}$ Common to supply the PLC modules as well as the LEDs.

Table D.3: Address Map for Problem in Part 1

\begin{tabular}{|c|c|c|}
\hline Address & Type & Description/Functional Requirement \\
\hline I:0/1 & External Input & ON/OFF Switch \\
\hline O:0/1 & $\begin{array}{c}\text { External } \\
\text { Output }\end{array}$ & Green LED - ON State \\
\hline O:0/3 & $\begin{array}{c}\text { External } \\
\text { Output }\end{array}$ & Red LED - OFF State \\
\hline T4:1 & Timer & Timer Address; Time Base - 1s \\
\hline
\end{tabular}

\section{D.3.1.2 Part 2 - Problem Description}

As an Engineering consultant for an Automobile company, you have been contracted to solve the following control problem. As part of a larger system, you have been provided a Motor starter and a cooling fan that continues to operate at certain times before it finally stops to dissipate the heat generated by the motor. The following specifications and constraints have been presented to you:

i) When the system is started, both the Motor and the Cooling Fan must be turned ON.

ii) On generating too much heat, the started switch goes OFF which turns on the Timer module. For the Timer, you have been given the following timing constraints:

a. The timer Preset is to be set for 60s. 
b. Fan must be off when $5 \mathrm{~s}<\mathrm{Tf}<=10$ s OR Tf $>=30$ s where $\mathrm{Tf}$ is the Fan Timer.

c. Fan must be $\mathrm{ON}$ during all other timing periods

iii) During the Timer periods, you are not allowed to turn on the switch again and the timer will continue to operate. Turning on the switch during these time periods will continue to keep the motor in OFF state. In all other periods, you can restart the Motor and the timer should reset back to 0 .

iv) In the event that the Timer module is done timing, you need to include a Reset Button to reset the timer so the Motor can be restarted.

Wire an 8 Input/8 Output PLC module in Automation Studio with a toggle switch to start \& stop the fan and motor and display the status of both i.e. whether it is ON or OFF using output LEDs. Use a $+24 \mathrm{~V}$ supply and 0V Common to supply the PLC modules as well as the LEDs.

Table D.4: Address Map for Problem in Part 1

\begin{tabular}{|c|c|c|}
\hline Address & Type & Description/Functional Requirement \\
\hline I:0/1 & External Input & ON/OFF Switch \\
\hline I:0/7 & External Input & Reset Pushbutton Button \\
\hline O:0/1 & $\begin{array}{c}\text { External } \\
\text { Output }\end{array}$ & Green LED - Motor ON State \\
\hline O:0/2 & $\begin{array}{c}\text { External } \\
\text { Output }\end{array}$ & Red LED \#1 - Motor OFF State \\
\hline O:0/4 & $\begin{array}{c}\text { External } \\
\text { Output }\end{array}$ & Yellow LED - Fan ON State \\
\hline O:0/5 & $\begin{array}{c}\text { External } \\
\text { Output }\end{array}$ & Red LED \#2 - Fan OFF State \\
\hline
\end{tabular}

Your logic should perform the operation in a way such as the timing diagram below. Use the 'Plotter' Function to obtain the timing diagram. Your timing diagram need not look the same but must behave similarly. 


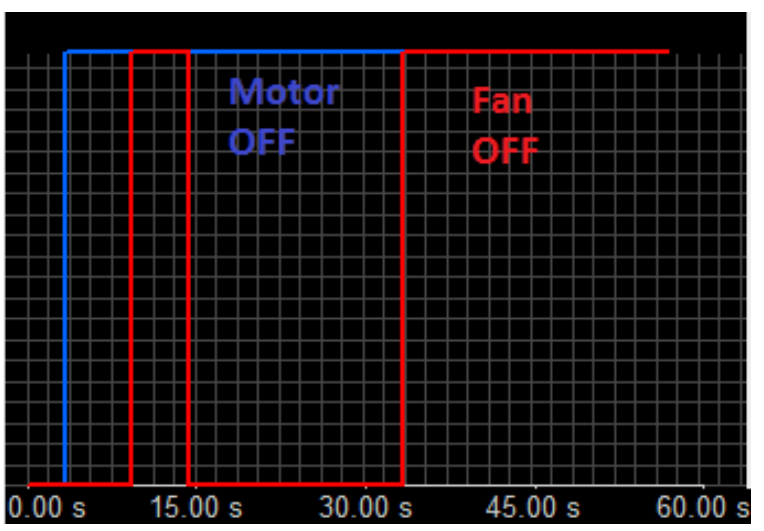

Figure D.9: Timing Diagram of OFF status LEDs

\section{D3.2 Laboratory Eight - Solution}

\section{D.3.2.1 Part 1 - Solution}
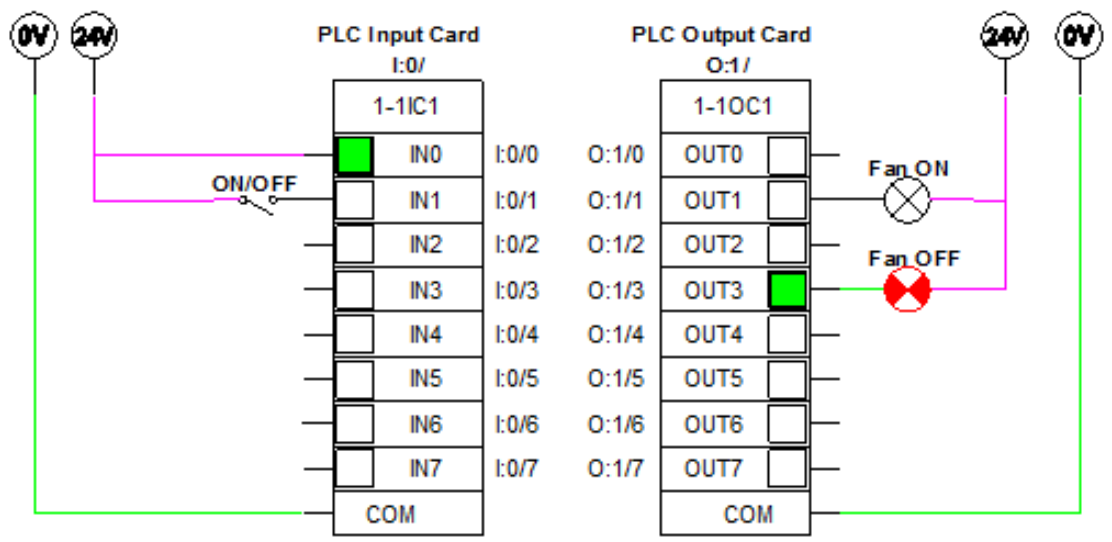

Figure D.10: I/O Wiring Diagram demonstrating the Fan in OFF state
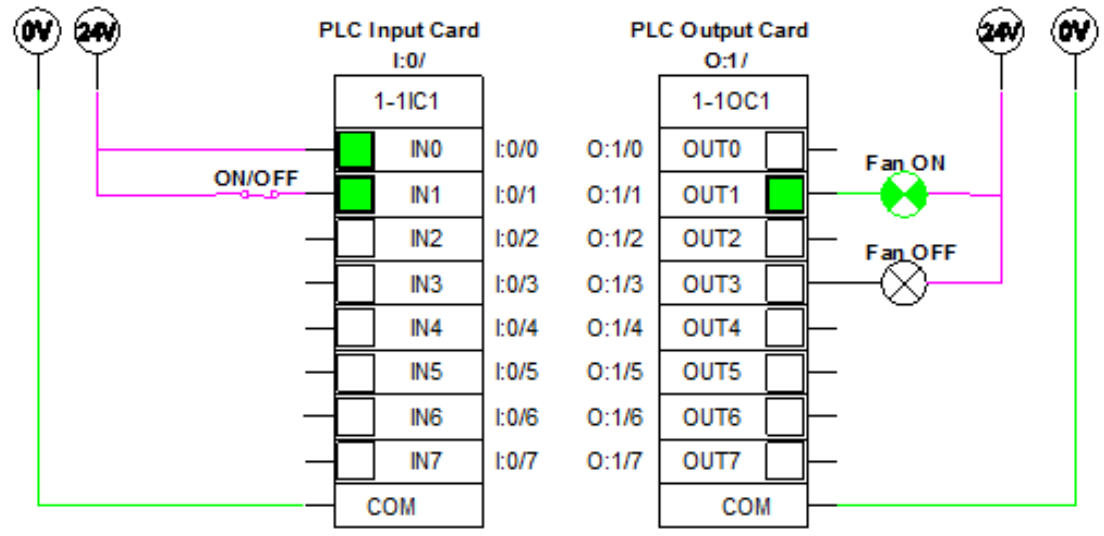

Figure D.11 I/O Wiring Diagram demonstrating the Fan in ON state 


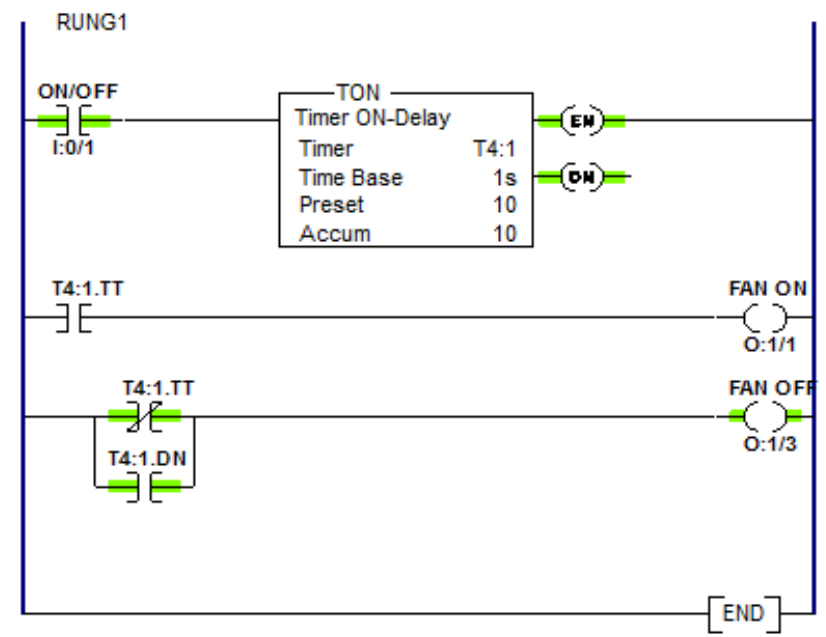

Figure D.12: Ladder Logic Diagram for Laboratory 4 - Part 1

\section{D.3.2.2 Part 2 - Solutions}
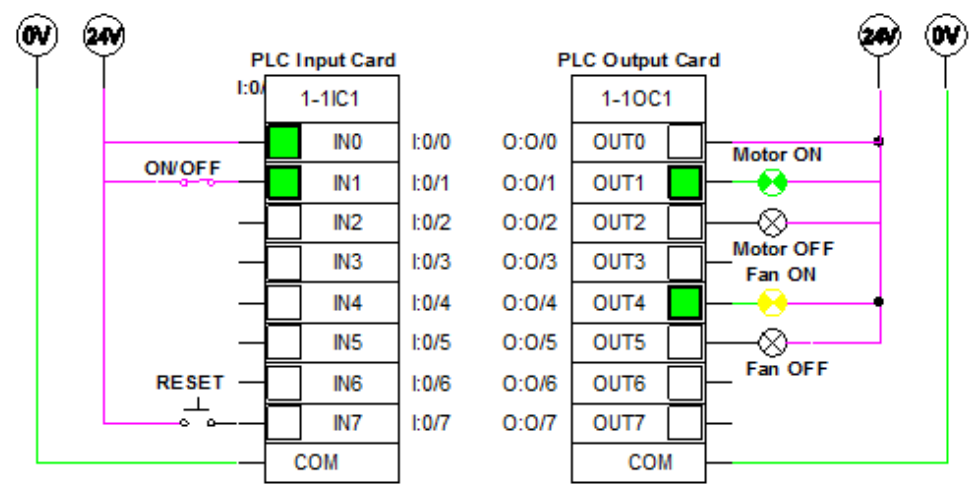

Figure D.13: I/O Wiring Diagram demonstrating the Motor and Fan in operation
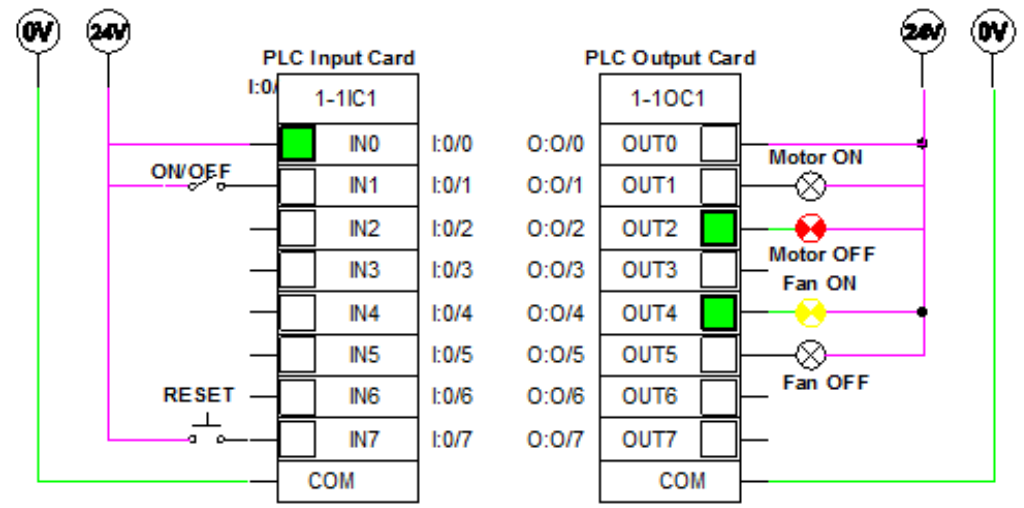

Figure D.14: I/O Wiring Diagram displaying the Motor OFF while the Fan continuing to operate 


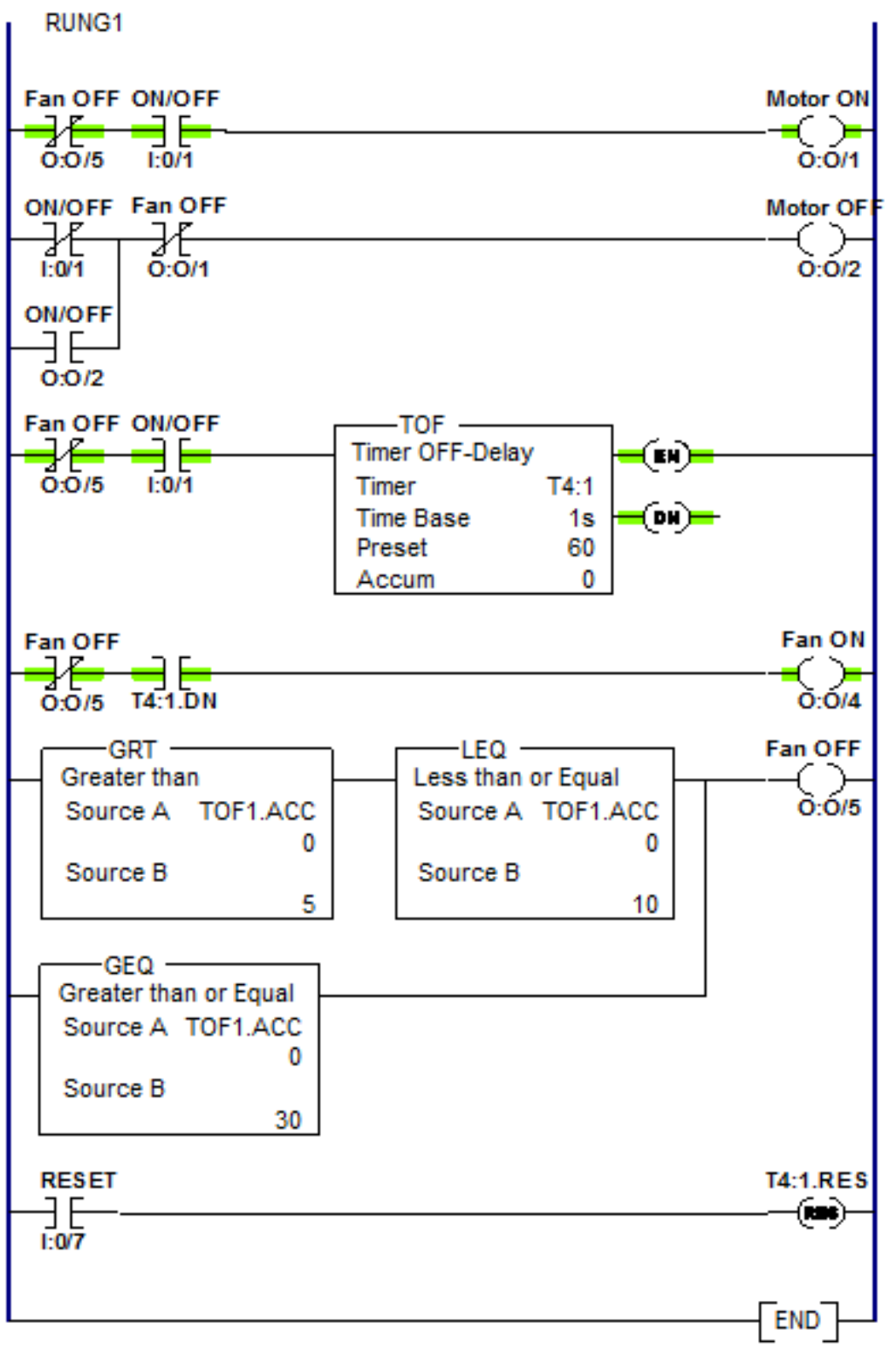

Figure D.15: Ladder Logic Diagram for Laboratory 4 - Part 2 


\section{Appendix E: Applications in the Steel \& Manufacturing Industries}

Manufacturing plants employ a large number of PLCs to significantly automate processes and increase the efficiency outputs of manufactured products. Here, concepts of 'Latching' and 'Interlocking' are very crucial to the applications in this industry to perform desired operation. The process of Latch/Unlatch is used to keep an output energized continuously even after an input is turned OFF. Interlocking method is used when there are multiple outputs that need to be controlled but only one of them should be $\mathrm{ON}$ at any given time i.e. switching the state of one output to ON must turn OFF the other outputs and so on.

Applications of their use can be found in the industry to control products conveying systems, sequencer systems, product sorting, shear press, die cutting as well as material positioning and handling. The following labs demonstrate such manufacturing operations that are commonly performed in the steel industry.

\section{Laboratory Nine: Conveyor Automatic Stacking}

\section{E.1.1 Laboratory Procedure}

A conveyor is a mechanical system commonly found in all industrial plants to automate processes. In this lab, you are required to write program the PLC to perform the conveyor operation depicted in the Figure below. The stacking system consists of 2 conveyor belts M1 and M2 along with a sensor. This sensor is used to count the transfer of each metal plate from conveyor M1 onto M2. Each time this stacking process occurs, the number of stacked metal plates is incremented. 


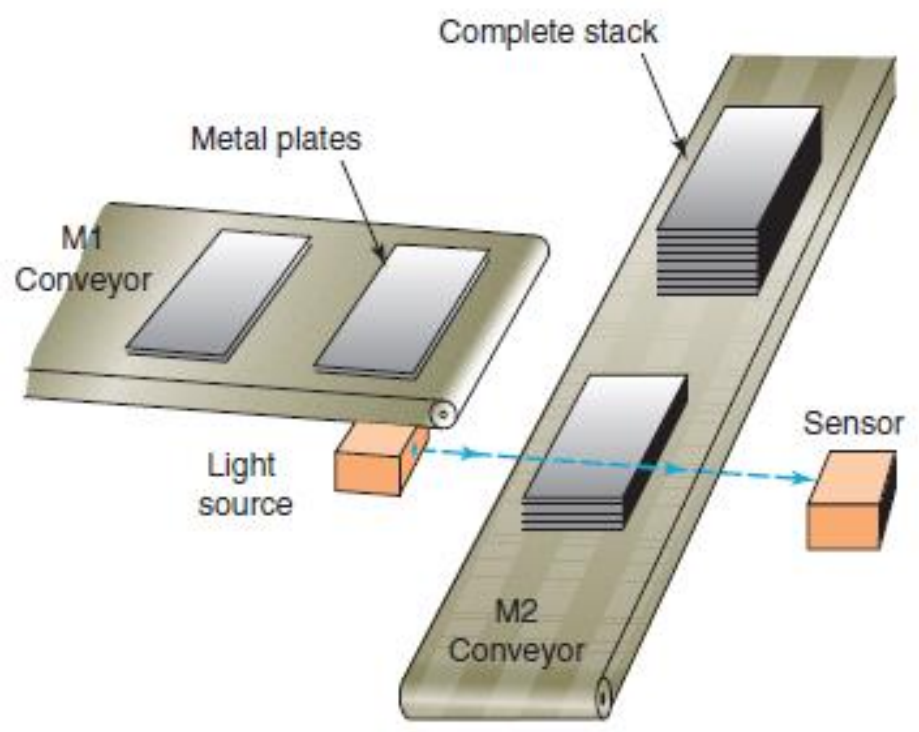

Figure E.1: Automatic Conveyor Stacking System [5]

The system operates under the following constraints:

i) When the system is started, the conveyor belt M1 begins to operate.

ii) The counter must increment upto a value of 10 i.e. when the sensor detects 10 plates transferred from M1 onto M2, the M1 conveyor belt must stop running and M2 must begin to operate.

iii) The M2 belt operates for $5 \mathrm{~s}$ following which the above sequence continues to operate.

iv) In order to restart the conveyor belt M1, the DN bit of the Timer and counter must be used.

Table E.1: Address Map for control problem in Lab 9

\begin{tabular}{|c|l|l|}
\hline Address & \multicolumn{1}{|c|}{ Type } & \multicolumn{1}{|c|}{ Description/Functional Requirement } \\
\hline I:0/1 & External Input & Pushbutton NC - Stop Operation \\
\hline I:0/2 & External Input & Pushbutton NO - Start Operation \\
\hline I:0/3 & External Input & $\begin{array}{l}\text { Toggle Switch NO - Manual Sensor } \\
\text { Detection }\end{array}$ \\
\hline O:0/1 & External Output & Indicator Light - Conveyor Belt M1 \\
\hline O:0/3 & External Output & Indicator Light - Conveyor Belt M2 \\
\hline
\end{tabular}


Using these specifications and the address map, provide a solution for the control problem using an 8 I/O PLC Module and make appropriate wirings for the power supply and ground.

\section{E.1.2 Laboratory Nine - Solution}
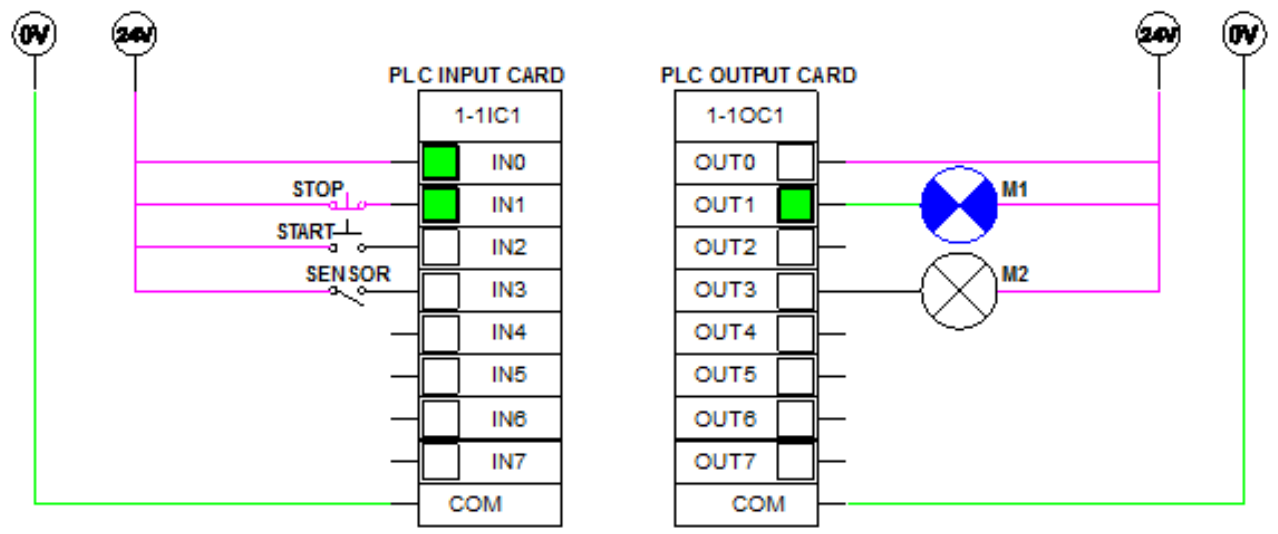

Figure E.2: PLC I/O Wiring Diagram for Lab 9 


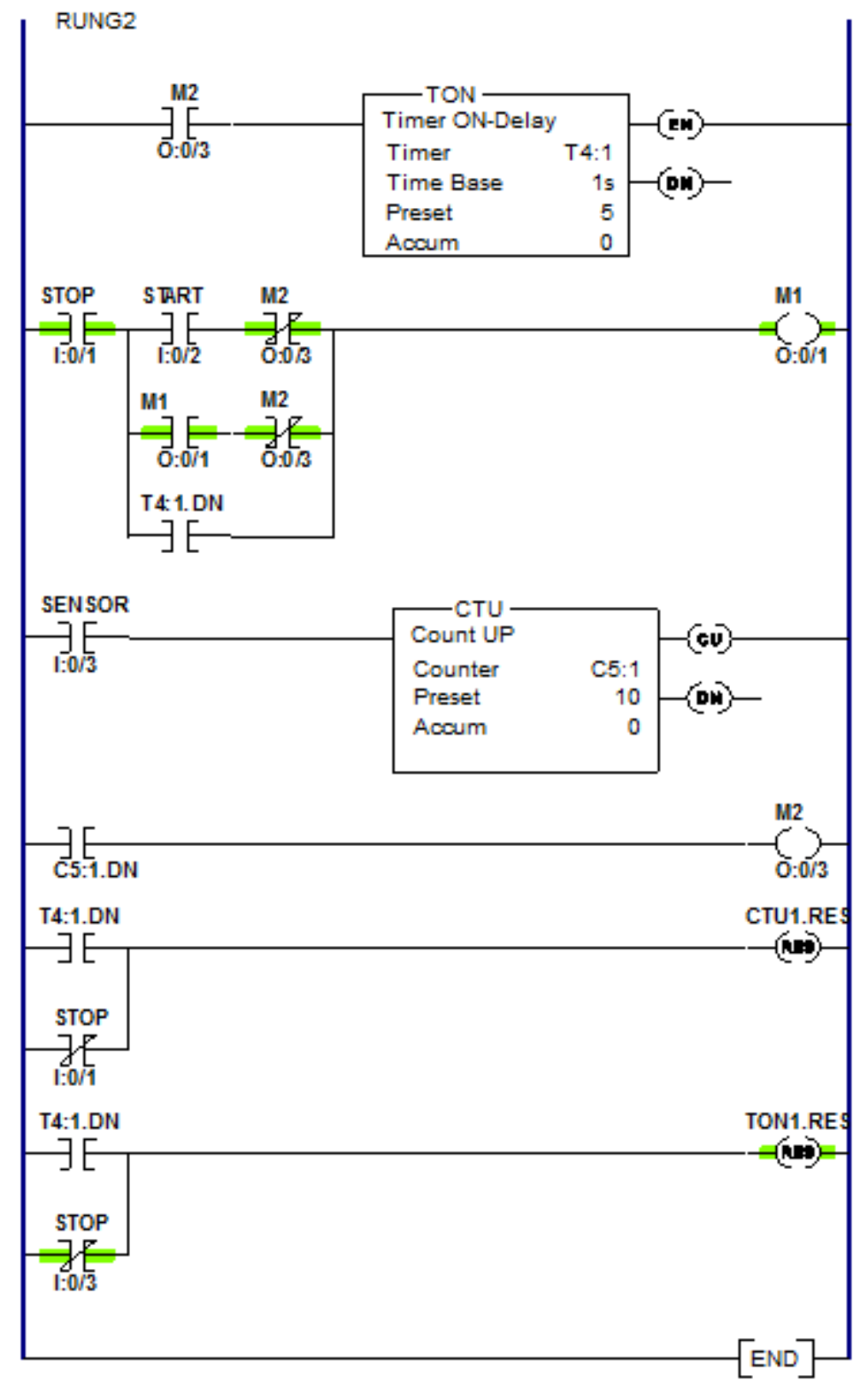

Figure E.3: Ladder Logic program for the Automatic Conveyor Stacking System 


\section{Laboratory Ten: Product Sorting}

\section{E2.1 Laboratory Procedure}

Product sorting is one such automated process that is routinely performed to distinguish good parts and bad parts. Similar to the previous laboratory, the conveyor here consists of a motor as well as a sensor. The objective of this control problem is to position the parts on the conveyor belt using the sensor in the system. In the event that the sensor detects a part, a timer is started for a period of $5 \mathrm{~s}$ following which the conveyor stops. Once the belt has been stopped, yet another timer is started for $5 \mathrm{~s}$ to restart the conveyor belt.

In order to sort the products into good or bad, gages have been incorporated in the system to distinguish between the parts and identify which ones hold defects. If a good part is detected, the system continues to operate as previously - continuously detecting parts with the timer delays. In the event that the bad part is detected, the timer delay is avoided by the system and a pneumatic cylinder begins to operate.

Table E.2: Address Map for control problem in Lab 10

\begin{tabular}{|c|l|l|}
\hline Address & \multicolumn{1}{|c|}{ Type } & \multicolumn{1}{c|}{ Description/Functional Requirement } \\
\hline I:0/1 & External Input & Pushbutton NC - Stop Operation \\
\hline I:0/2 & External Input & Pushbutton NO - Start Operation \\
\hline I:0/3 & External Input & Toggle Switch NO - Part Detection \\
\hline I:0/4 & External Input & Toggle Switch NC - Part Good Status \\
\hline O:0/1 & External Output & Indicator Light - Status Light \\
\hline O:0/3 & External Output & Indicator Light - Motor Status \\
\hline O:0/5 & External Output & Indicator Light - Cylinder Status \\
\hline
\end{tabular}

Using these specifications and the address map, provide a solution for the control problem using an 8 I/O PLC Module and make appropriate wirings for the power supply and ground. 


\section{E2.2 Laboratory Ten - Solution}
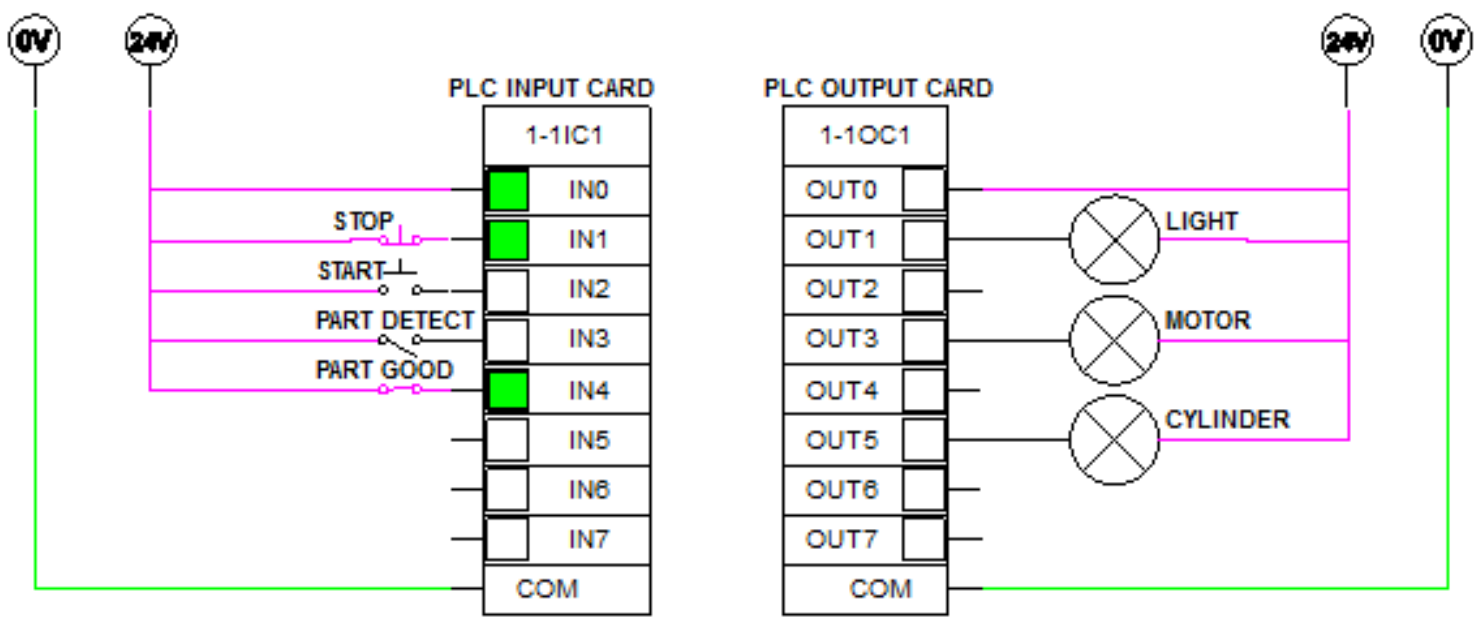

Figure E.4: PLC I/O Wiring Diagram for the Product sorting system

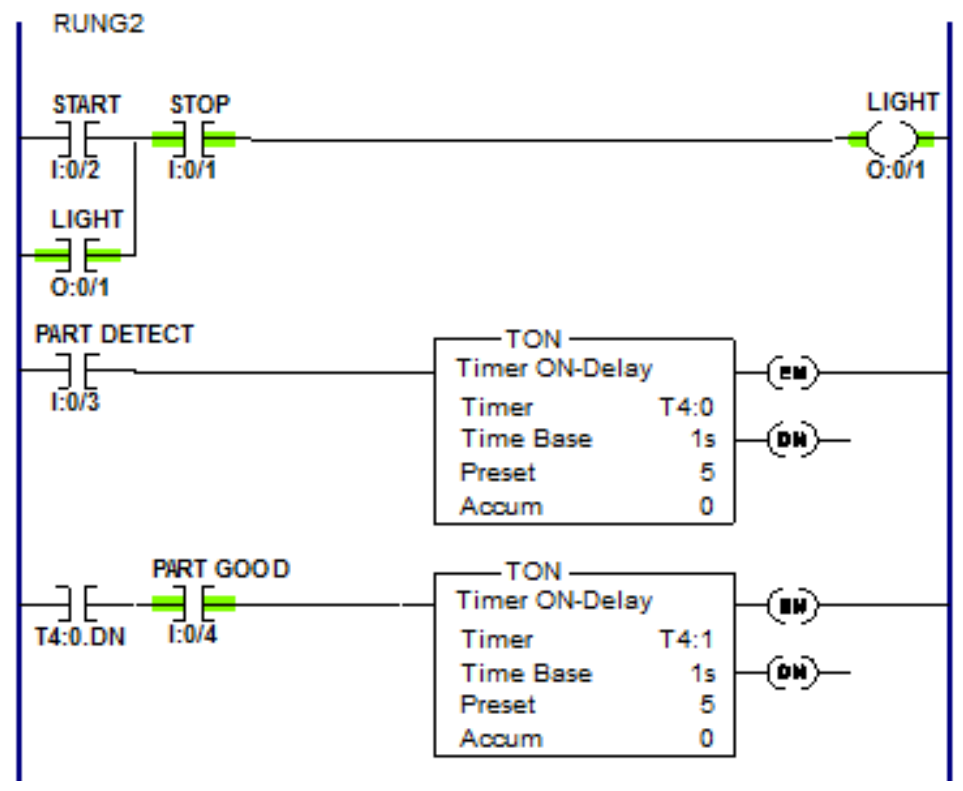

Figure E.5: Part 1 of the Ladder Logic program for the Product sorting system 


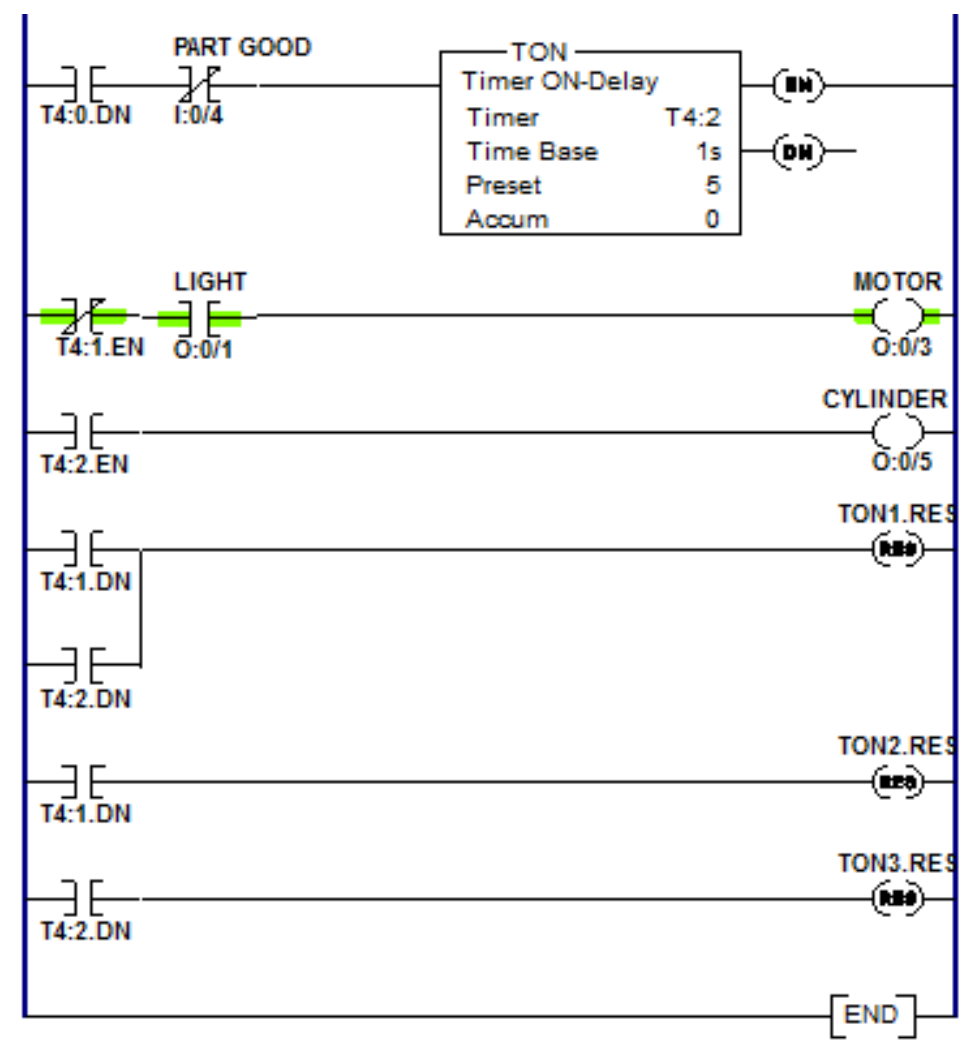

Figure E.6: Part 2 of the Ladder Logic program for the Product sorting system

\section{Laboratory Eleven: Shear Press}

\section{E3.1 Laboratory Procedure}

One of the most common operations in the steel industry is the process of die cutting, also known as Shear Press. The process is essentially the method by which sheet metals are cut and new parts and materials are produced using shear force. Parts produced are referred to as 'Stamped' components and are a result of different shear press processes like blanking and drawing. Examples of these Stamped materials or parts can be found all around us such as in canned drinks, automobile parts to household door mechanisms and other structural parts.

In this lab, you are required to program the die cutting machine to control a solenoid cylinder that extends to stamp materials and retracts. The solenoid can often be any actuator that needs to be controlled based on the type of shearing process and programming requirements. 


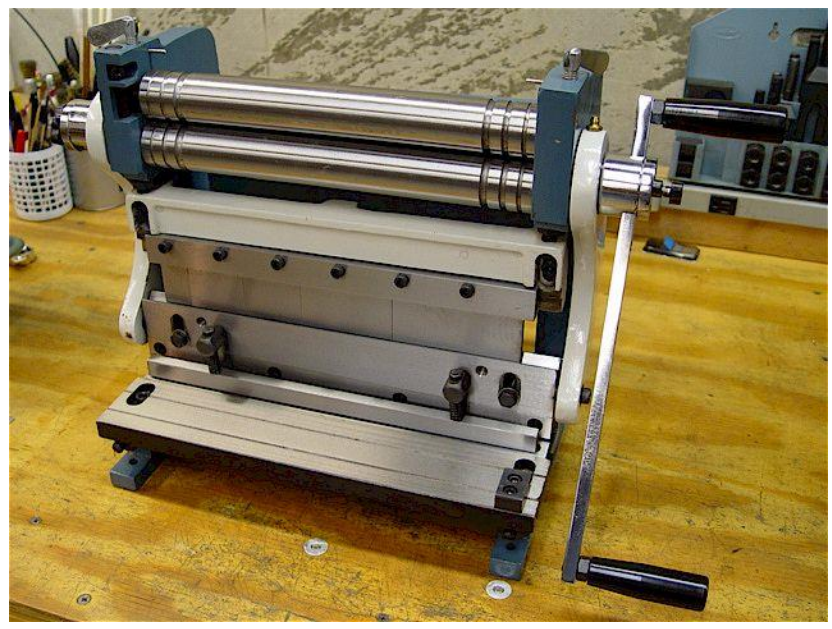

Figure E.7: Shear Press system [29]

The machine works with the following constraints:

i) In order to turn on or energize the solenoid (SOL), both the start switch (TS) and safety switch (LS1 - for Limit Switch) must be turned ON.

ii) On activating the SOL, it continues to remain ON until another safety switch (LS2) is activated. Turning $\mathrm{ON}$ of this second switch indicates that the stamping process is complete which is when the cylinder must retract from its extended position (i.e. the SOL must turn OFF).

iii) Completion of the extension and retraction of the cylinder results in a third limit switch (LS3) to turn ON. If the switch is not ON, the cycle should be unable to restart.

iv) The system must include a safety feature to alert the operator when the machine is jammed or faulty. You are required to include a Timer module (Preset: 7 seconds) and an output indicator LIGHT to demonstrate this.

v) The ladder program must also be able to count the number of parts produced. Each time 7 parts have been produced, the machine must come to a complete stop. Also, include a RESET feature to reset the counter once complete. 
Table E.3: Address Map for control problem in Lab 11

\begin{tabular}{|c|l|l|}
\hline Address & \multicolumn{1}{|c|}{ Type } & \multicolumn{1}{|c|}{ Description/Functional Requirement } \\
\hline I:0/1 & External Input & Toggle Switch NO - TS1 \\
\hline I:0/2 & External Input & Toggle Switch NO - LS1 (Switch 1) \\
\hline I:0/3 & External Input & Toggle Switch NO - LS2 (Switch 2) \\
\hline I:0/4 & External Input & Toggle Switch NO - LS3 (Switch 3) \\
\hline I:0/5 & External Input & Pushbutton NO - Reset \\
\hline O:0/1 & External Output & Indicator Light - Solenoid Status \\
\hline O:0/5 & External Output & Flashing Coil - Alarm (LIGHT) \\
\hline
\end{tabular}

Using these specifications and the address map, provide a solution for the control problem using an 8 I/O PLC Module and make appropriate wirings for the power supply and ground.

\section{E3.2 Laboratory Eleven - Solution}
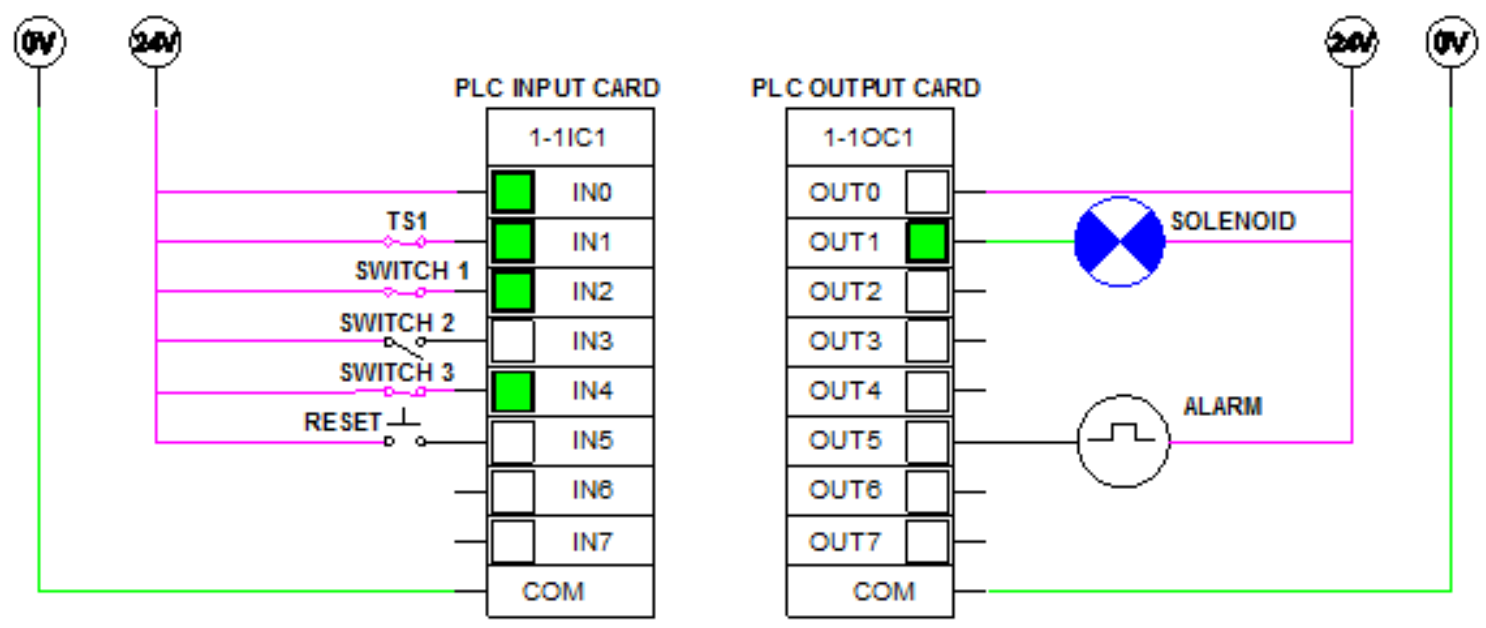

Figure E.7: PLC I/O Wiring Diagram for Laboratory 11 


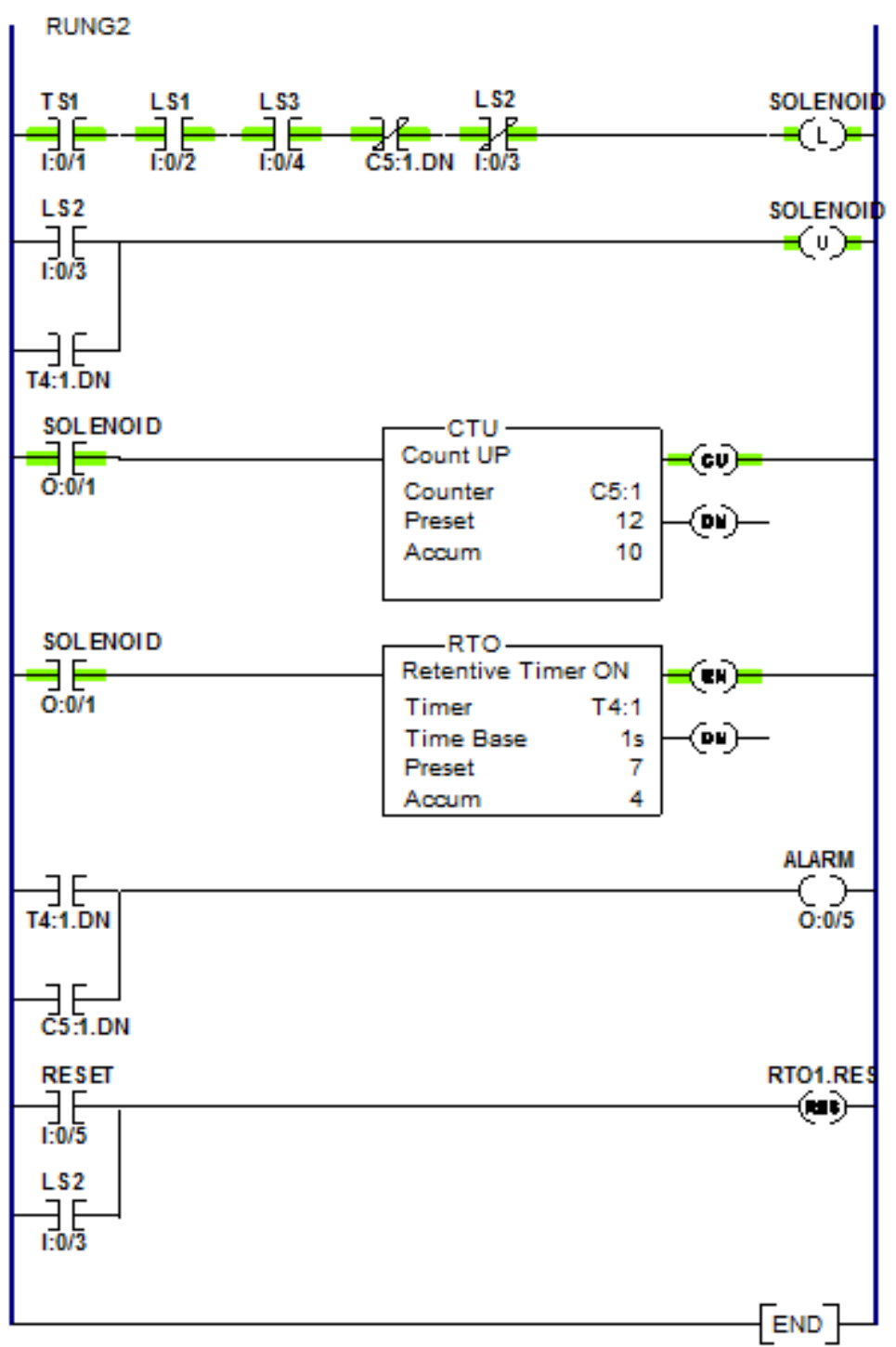

Figure E.6: Ladder Logic program for Laboratory 11 


\section{Appendix F: Applications in Power \& Gas Industries}

\section{Laboratory Twelve: Control of Pneumatic Cylinders}

In this controls lab, we look into pneumatic cylinders that are widely used in all industries such as automobile, healthcare and oil and gas. Pneumatic cylinders are simply mechanical devices work together to use compressed gas and produce a force. Examples of these are air brake systems, pressure regulators, compressed air engines and even some exercise machines [14]. Thus, pneumatic cylinders use air pressure to produce a linear motion that can be optimized and used to perform a desired operation [14].

Students are expected to review their knowledge on Pneumatic cylinder and hydraulics as well as review Counters and Timer functions from Lab 1. In this lab, we will learn to control a double-acting Pneumatic cylinder using PLCs and clock the time of failure events that occur during its operation.

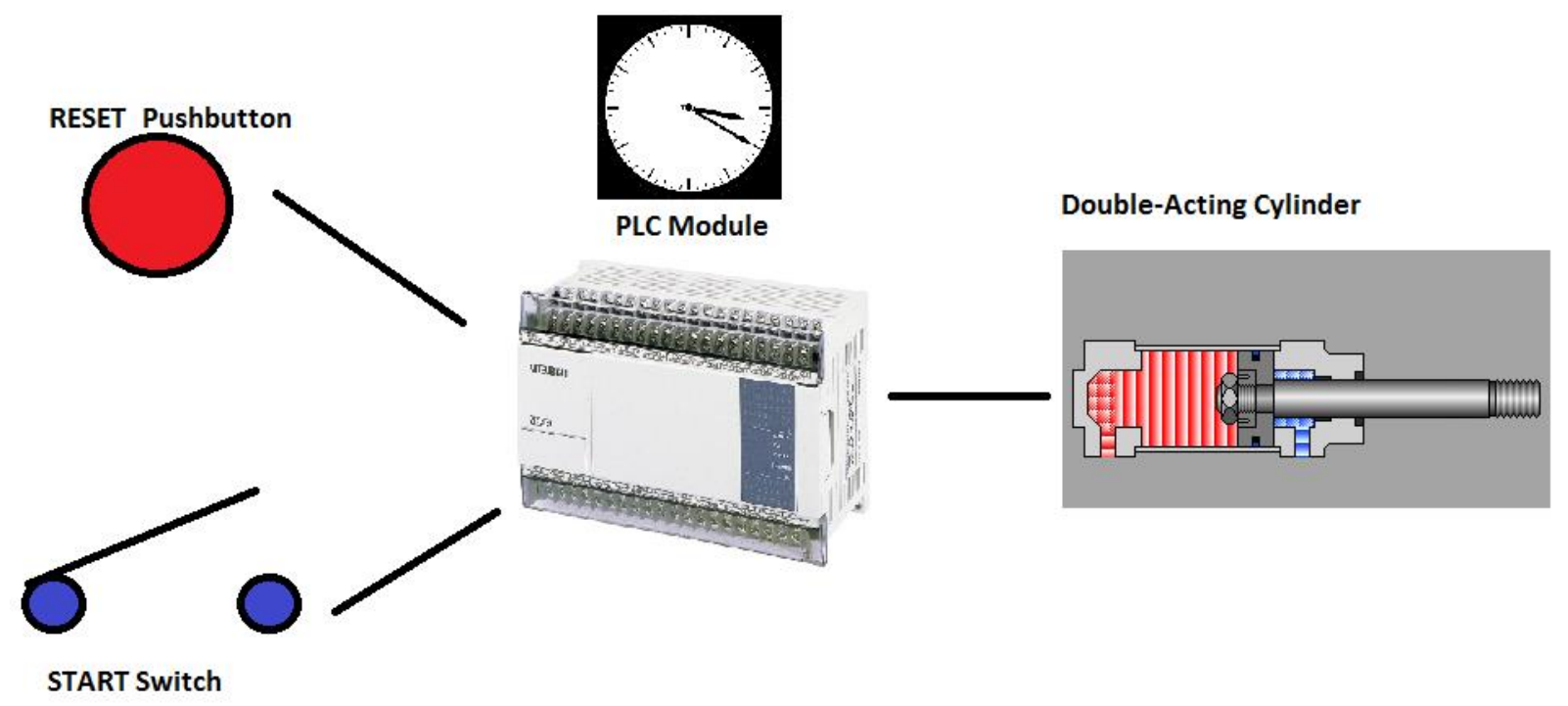

Figure F.1: Visualization of Control problem for Laboratory 5 


\section{F1.1 Laboratory Procedure}

\section{E.2.1 Problem Description}

You are required to control a double-acting cylinder that is constantly operating and clock the timing in the event that the circuit fails or trips. You are to record the timing of three failure events before completely shutting off the system and raising an alarm to change the device. The control problem has the following constraints:

vii. Once the system is turned ON (i.e. you begin the simulation), the cylinder only begins to operate when the reset button is pressed.

viii. The timing of the event must be in seconds, minutes and hours.

ix. In the event that the circuit trips (when the switch is open at any time), the timing must automatically stop and indicate this with an LED.

x. You are allowed to turn $\mathrm{ON}$ the switch up to 3 times before sending out an alarm to change the device. In this event, you will no longer be allowed to reset the timers or restart the clock and the system must be locked out.

You are required to appropriately determine the counters and timers you require to record the time in seconds, minutes and hours. Timing parameters can be reduce to demonstrate the appropriate functioning of the logic and lab objectives

Design a ladder logic program to clock the timing of a failure event as well as satisfying all the constraints above. Also, wire the Pneumatic cylinder and link it to the ladder logic program using a Solenoid. Since the inputs and outputs are less, 8 point input module and 8 point output module is sufficient using the $+24 \mathrm{~V}$ and ground supplies on Automation Studio.

Table F.1: Address Map for Problem in Part 1

\begin{tabular}{|c|c|c|}
\hline Address & Type & Description/Functional Requirement \\
\hline I:0/1 & External Input & Start - Normally Closed Switch \\
\hline I:0/2 & External Input & Reset - Normally Open Pushbutton \\
\hline B3:0/0 & Internal & Internal Memory Bit \\
\hline O:0/3 & External Output & Circuit Tripped - Status indicated by LED \\
\hline O:0/6 & External Output & Change Device - Blinking LED \\
\hline O:0/7 & External Output & Solenoid - connected to the Pneumatic Circuit \\
\hline
\end{tabular}




\begin{tabular}{|c|l|l|} 
T4:1 & RTO Timer & To count the seconds of failure event \\
\hline T4:2 & TON Timer & $\begin{array}{l}\text { Timer for the Pneumatic Cylinder to operate } \\
\text { continuously }\end{array}$ \\
\hline C5:1 & Counter Up 1 & To count the minutes of failure event \\
\hline C5:2 & Counter Up 2 & To count the hours of failure event \\
\hline C5:3 & Counter Down & To count the number of times circuit has been Reset \\
\hline
\end{tabular}

\section{F1.2 Laboratory Twelve - Solutions}

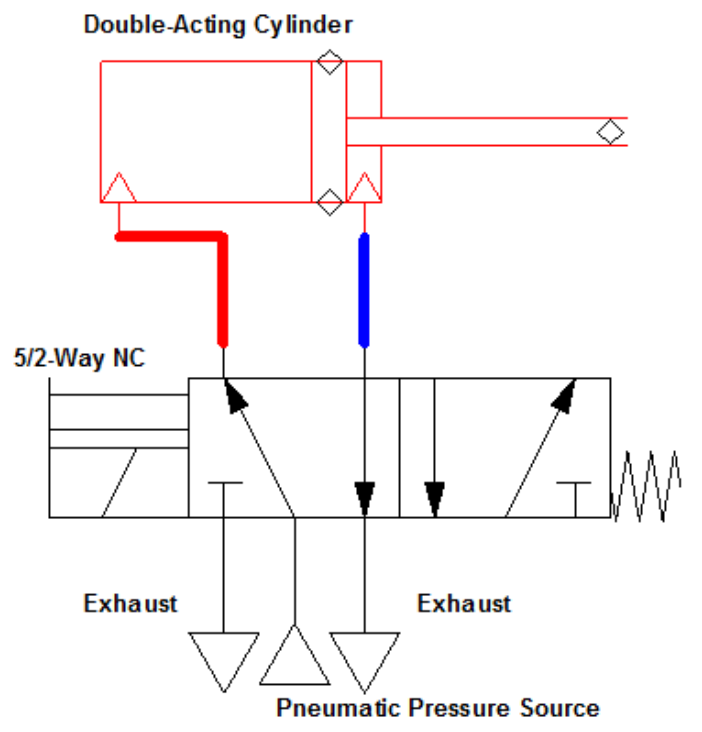

Figure F.2: Wiring Connections to the Double Acting Cylinder to begin operation 


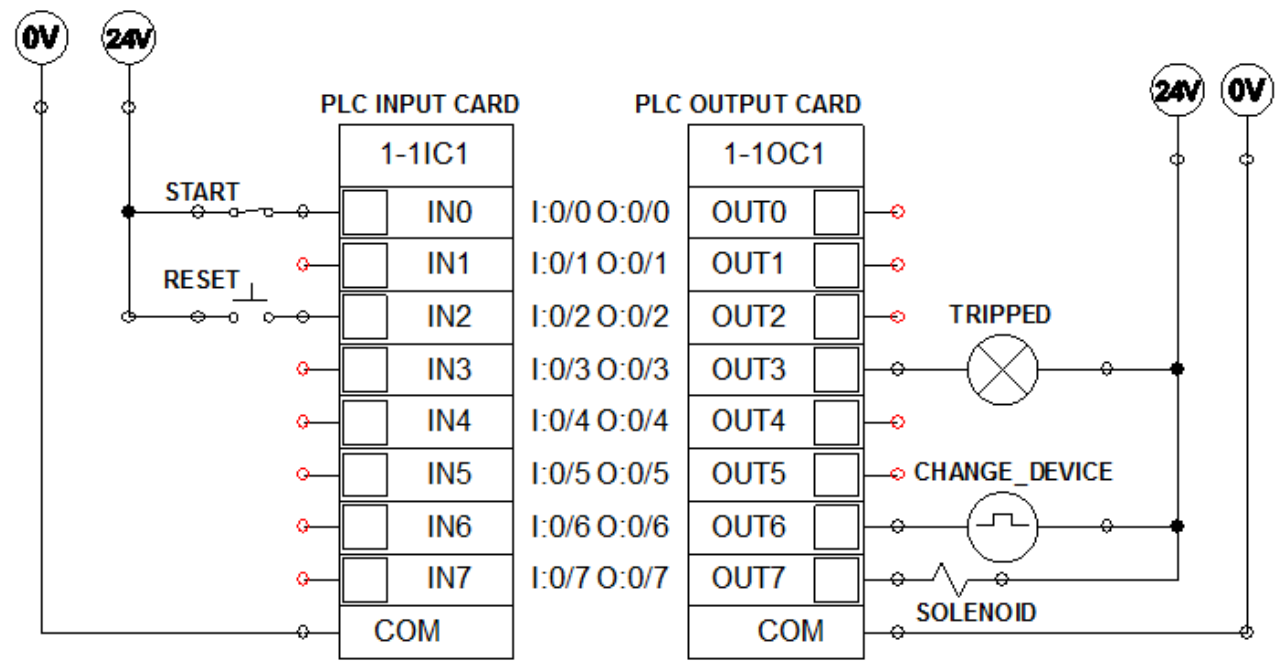

Figure F.3: I/O Wiring Diagram to the PLC Module for Lab 5

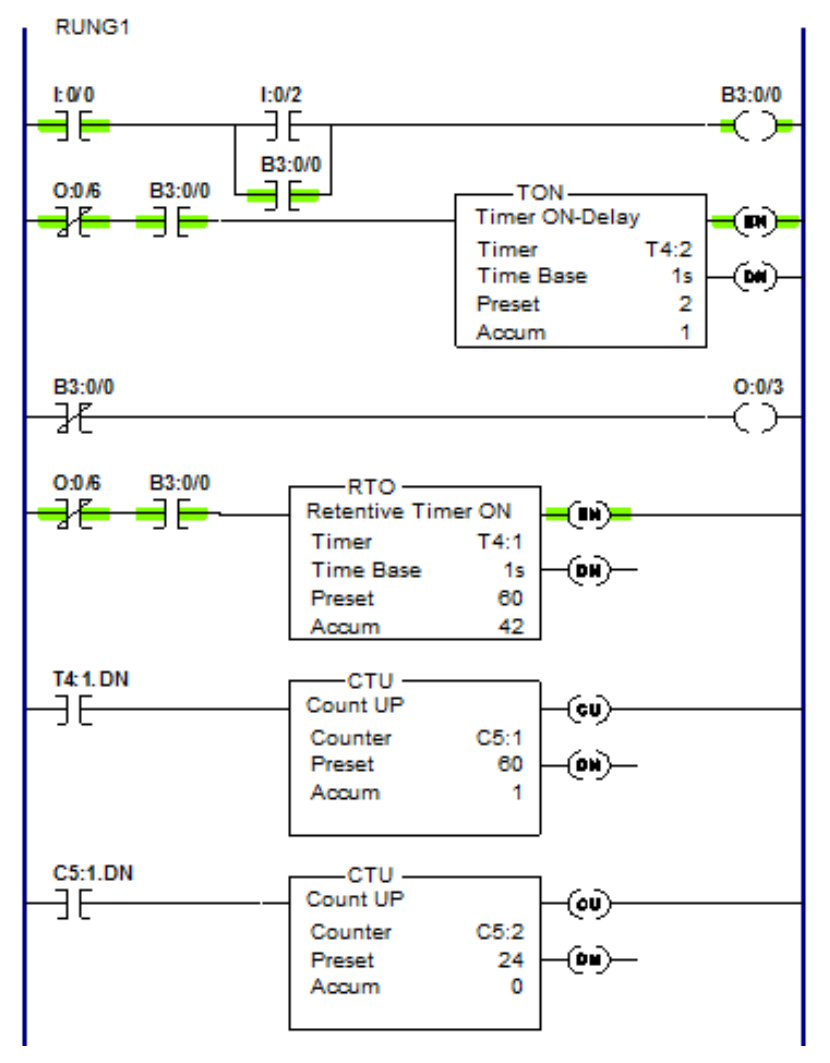

Figure F.4: Ladder Logic Diagram Part $1\left(1^{\text {st }}\right.$ Failure Event Clocked at $\left.\mathrm{t}=00: 01: 42\right)$ 


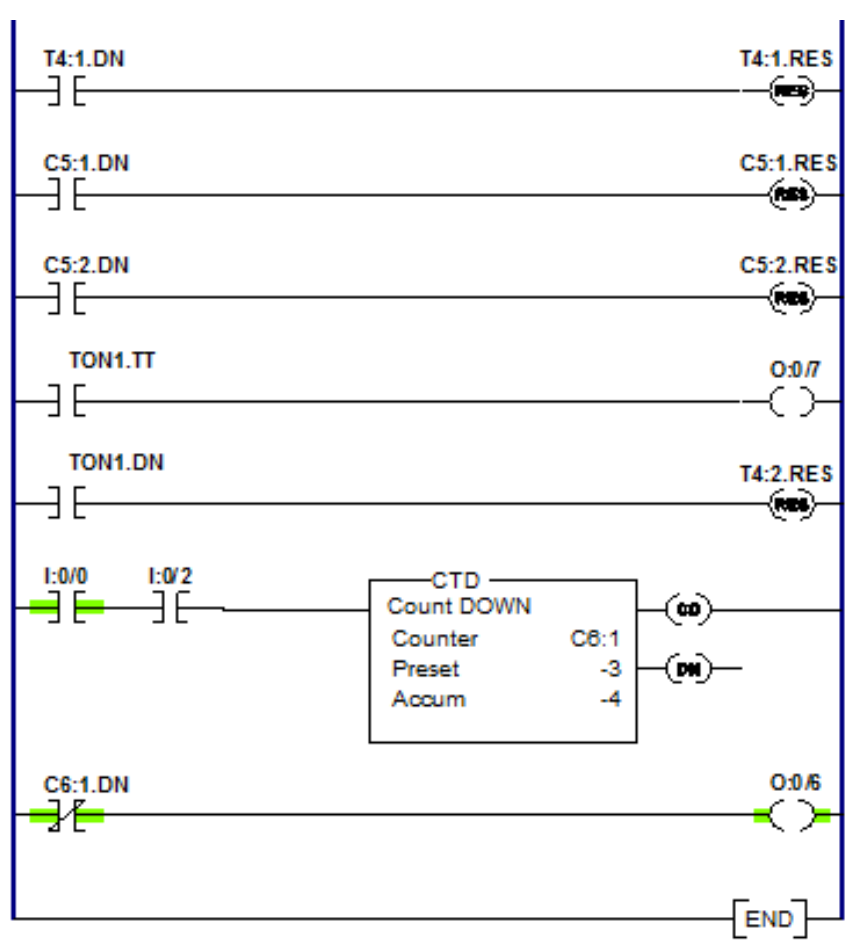

Figure F.5: Ladder Logic Part 2 (Reset Timers and Alarm Activated) 


\section{Laboratory Thirteen: Directional Control of}

\section{Motors}

The goal of this lab is to solve the Motor Control problem and understand how PLCs can be used to control and operate motors as desired. Students are expected to review their knowledge on Motor Theory and the types of timers and how they function in ladder logic. For this lab you will be required to control a Motor in Forward (clockwise) and Reverse (Anticlockwise) directions along with a coolant pump that is actuated and turns off the motor if it continues to operate for an extended period. You will be required to use the inputs/outputs, address map, design guidelines provided as well as wire the PLC to achieve this objective.

\section{F2.1 Laboratory Procedure}

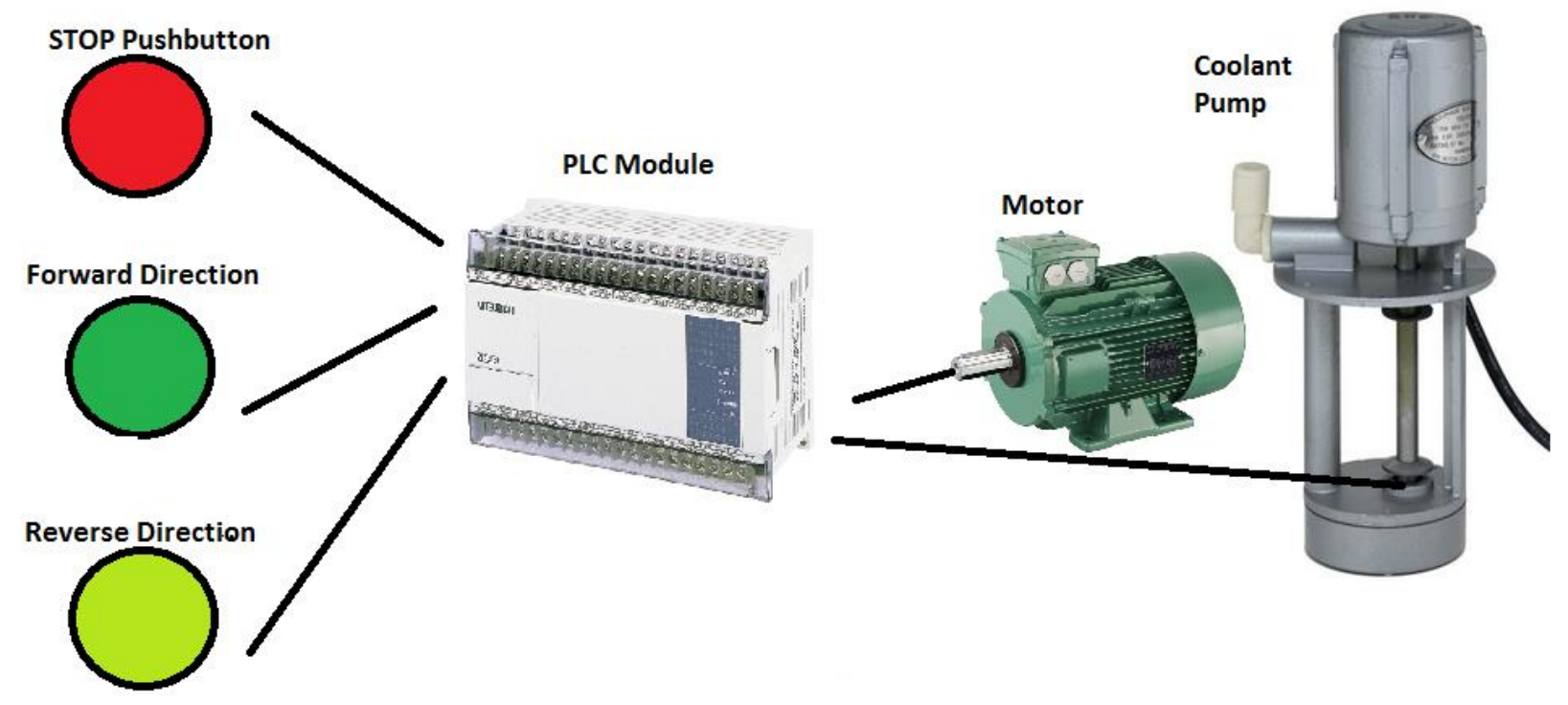

Figure F.6: Visualization of the Control Problem

\section{F.2.1 Problem Description}

You are required to control the direction of a motor using the PLC connected to 2 circuits - a Motor forward circuit (Forward Motor) and Motor reverse circuit (Reverse Motor). Along with the motor, there is also a coolant which needs to be running simultaneously. 
The control problem has the following constraints:

i. Once the system is turned ON (i.e. you begin the simulation), motor must be given 5 seconds before either Forward or Reverse Directions can be selected.

ii. On hitting either the Forward or Reverse Pushbutton, the motor \& the coolant pump must begin simultaneous operation

iii. The motor must be given a 5 second delay to slow down before the direction can be switched. If the motor is on Forward mode, hitting the Reverse pushbutton will first cause the motor to slow down followed by automatic operation in the Reverse direction.

iv. In the event that motor direction is not changed for 10 seconds, the coolant pump and the motor must both stop (the pump should stop instantly whereas the motor will take 5 seconds to slow down eventually to a stop).

v. Finally, pushing the STOP pushbutton should cause the motor to slow down and stop at any given time.

You are required to appropriately determine the types and number timers required to achieve this objective. Hint: The objective can be achieved with 3 timers for which you can use the file addresses as T4:0, T4:1, and T4:2.

Design a ladder logic program to change the direction of the motor as well as satisfying all the constraints above. Since the inputs and outputs are less, 8 point input module and 8 point output module is sufficient using the $+24 \mathrm{~V}$ and ground supplies on Automation Studio.

Table F.2: Address Map for Lab 13

\begin{tabular}{|c|c|c|}
\hline Address & Type & Description/Functional Requirement \\
\hline I:0/1 & External Input & Stop - Normally Open Pushbutton \\
\hline I:0/2 & External Input & Forward - Normally Open Pushbutton \\
\hline I:0/3 & External Input & Reverse - Normally Open Pushbutton \\
\hline O:0/0 & Internal Memory \#1 & Temporary Variable \#1 - Status indicated by LED \\
\hline O:0/1 & $\begin{array}{c}\text { Temporary Variable } \\
\text { \#1 }\end{array}$ & Motor Forward Circuit - Status LED \\
\hline O:0/2 & External Output & Motor Running in Forward Direction - LED \\
\hline O:0/3 & Internal Memory \#2 & Temporary Variable \#2 - Status indicated by LED \\
\hline O:0/4 & Temporary Variable & Motor Reverse Circuit - Status LED \\
\hline
\end{tabular}




\begin{tabular}{|c|c|c|} 
& $\# 2$ & \\
\hline O:0/5 & External Output & Motor Running in Reverse Direction - LED \\
\hline O:0/6 & External Output & Coolant Pump - Status LED \\
\hline O:0/7 & External Output & $\begin{array}{c}\text { Slow Down Blinking Light } \\
\text { - Blinks when motor is slowing down }\end{array}$ \\
\hline
\end{tabular}

\section{F2.2 Laboratory Thirteen - Solutions}
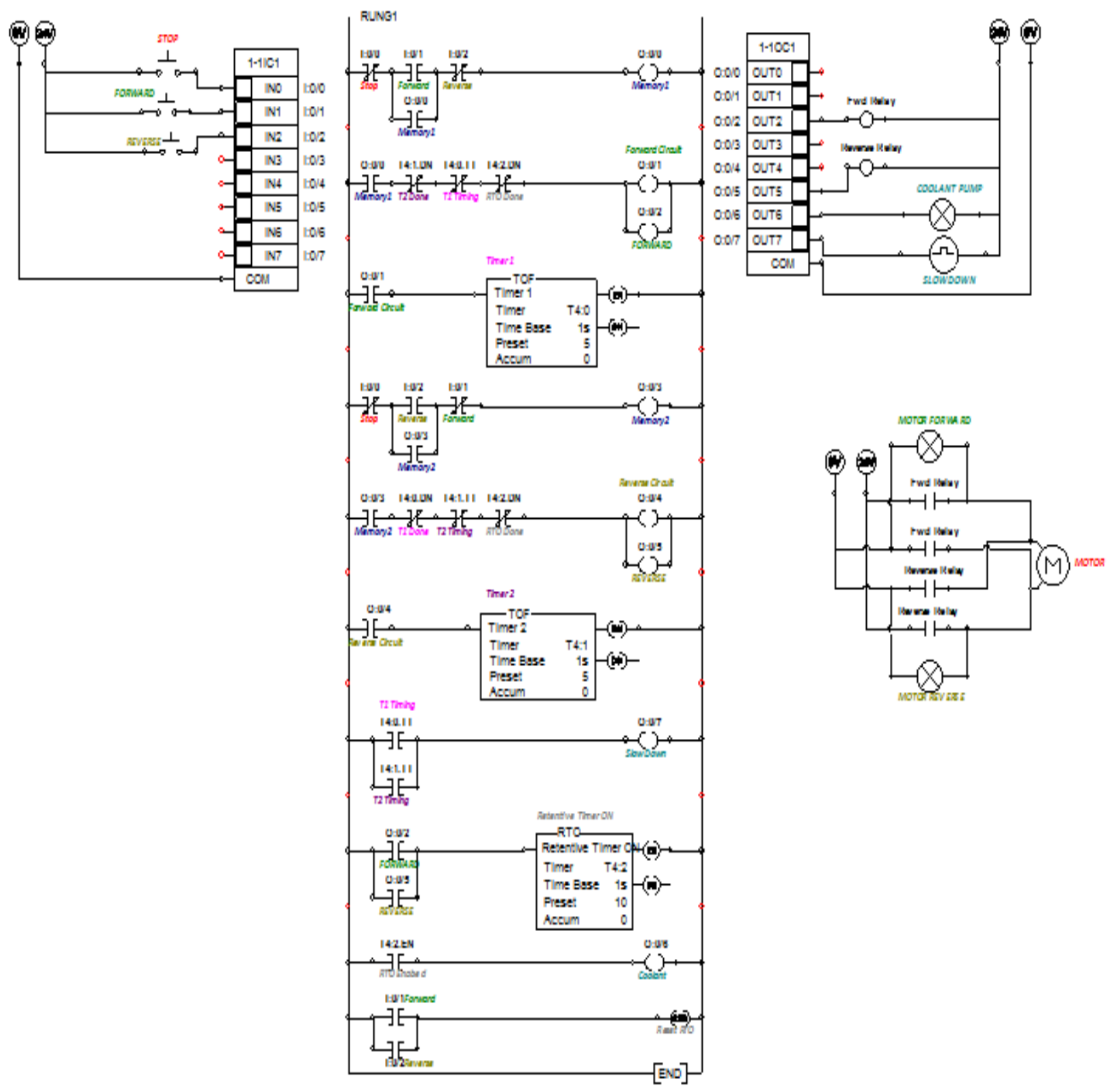

Figure F.7: Complete Solution for Laboratory 6 including

Wiring Diagrams and Ladder Logic 


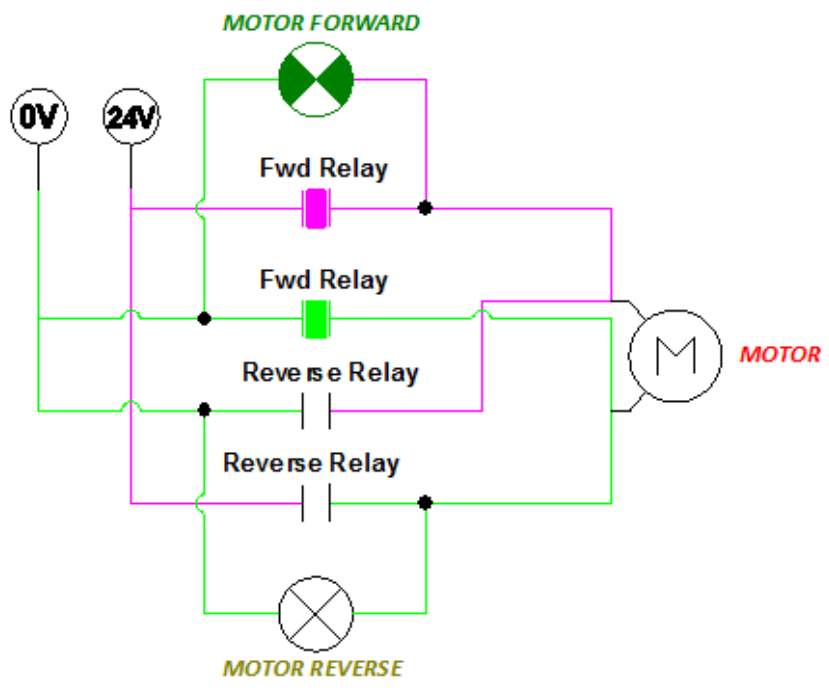

Figure F.8: Motor operating in FORWARD operation

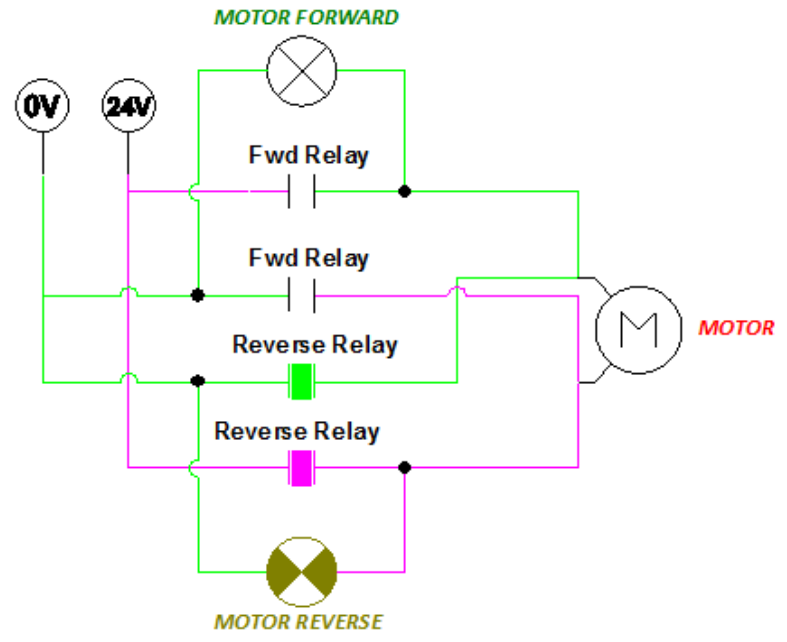

Figure F.9: Motor operating in REVERSE operation 

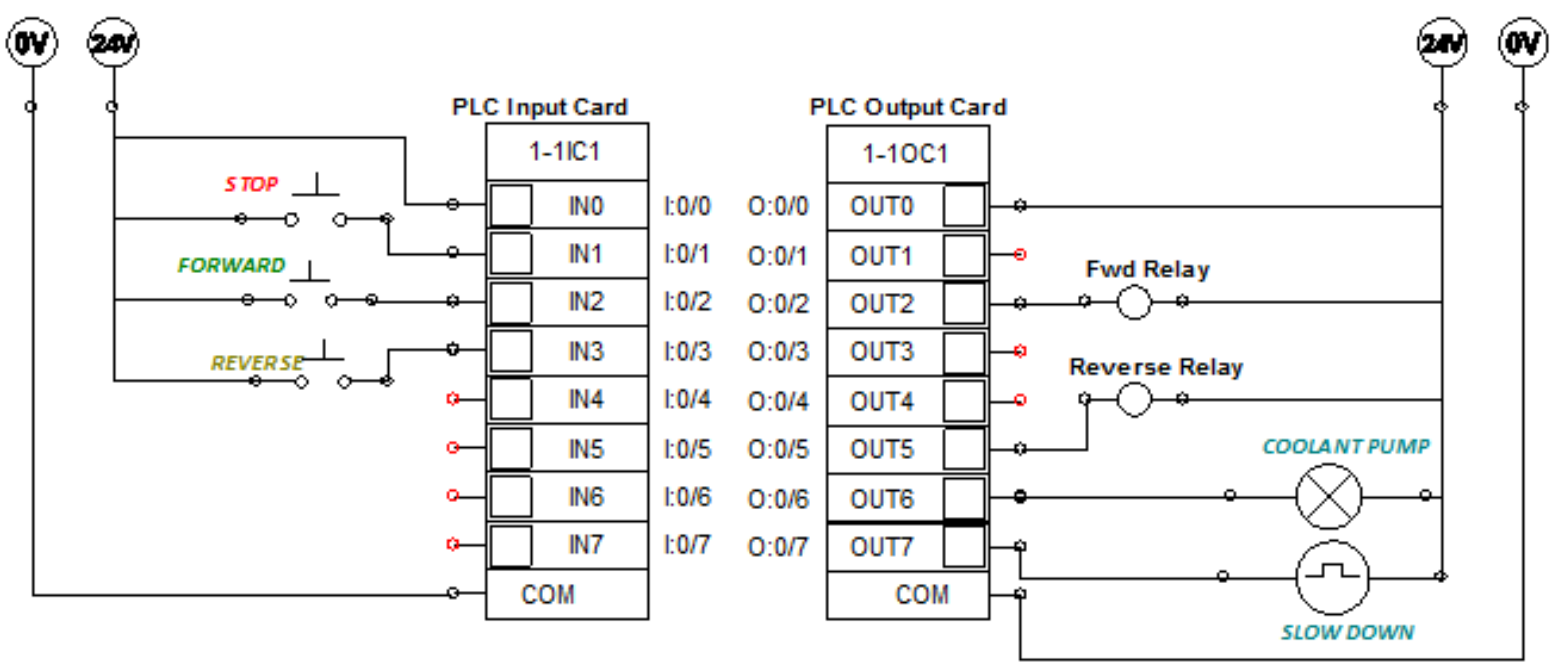

Figure F.10: I/O Wiring Diagram to the PLC module for Laboratory 6

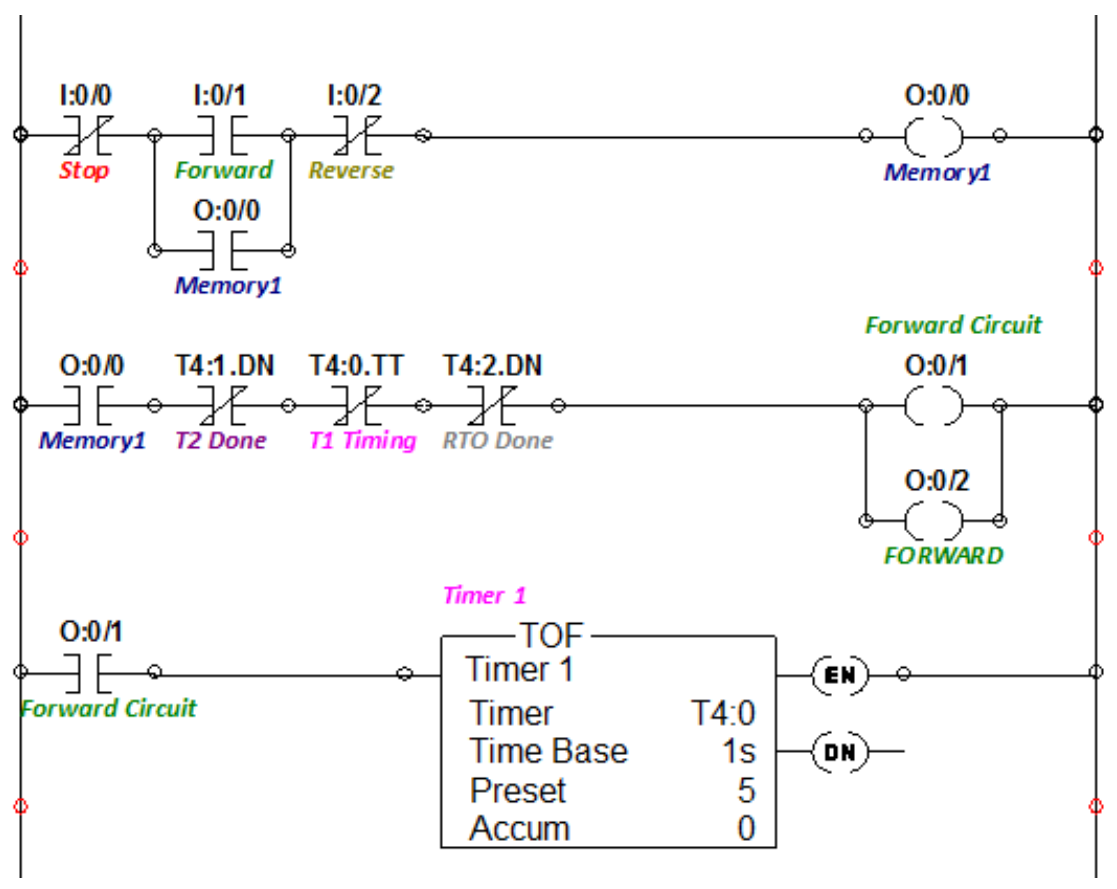

Figure F.11: Ladder Logic Diagram for Motor Forward Operation 


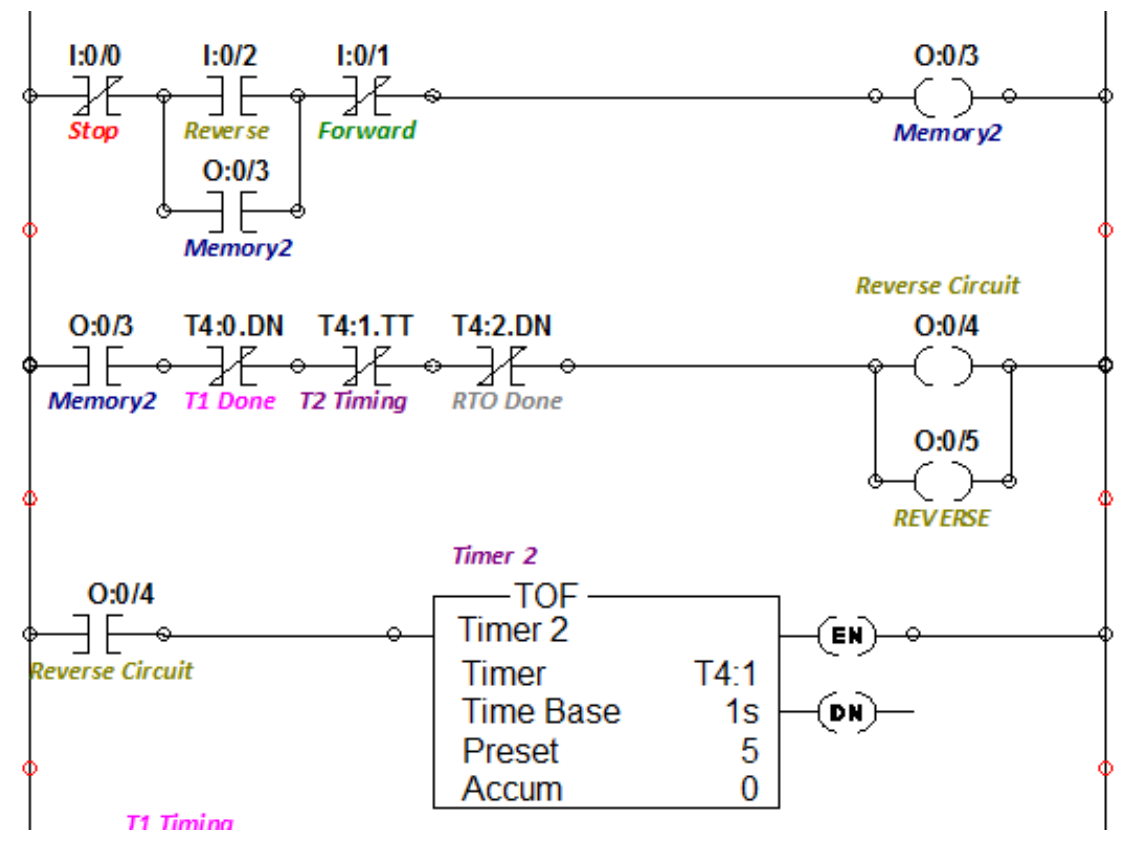

Figure F.12: Ladder Logic Diagram for Motor Reverse Operation

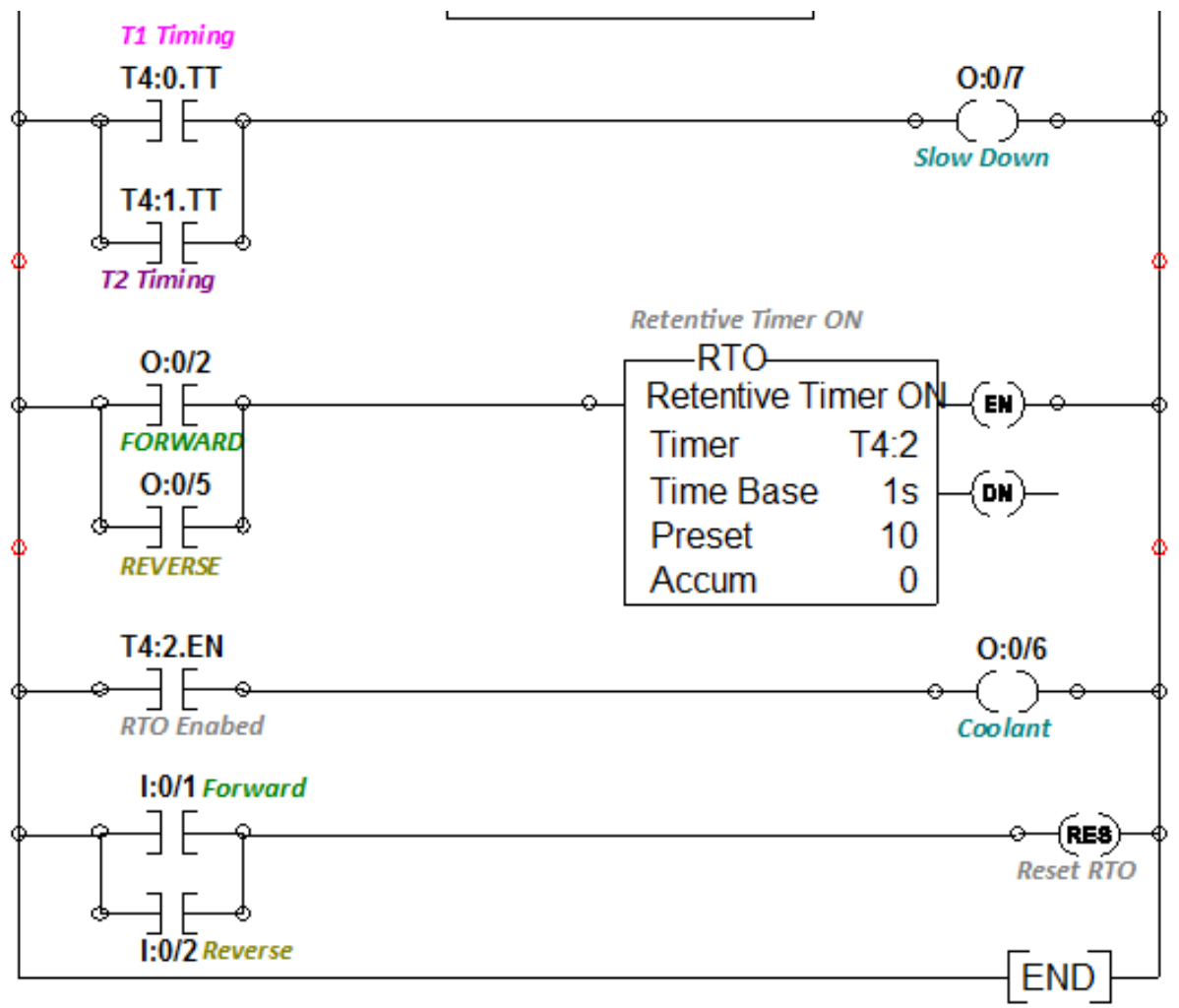

Figure F.13: Retentive Timer Logic Design \& Reset Operation 


\section{Laboratory Fourteen: Wind Turbine}

\section{Monitoring}

\section{F3.1 Laboratory Procedure}

The power and electricity demands are fast increasing with the increasing industrialization and urbanization around the globe. In today's times, electricity is one of the fundamental needs of many livelihoods that needs to be sustained and developed to continue life as we know it. One of vastly developed sources of electricity generation are Wind Turbines. Over time, these sources have been engineered and developed to a great extent to provide us with a renewable source of electricity supply.

As we know, Wind turbines are devices that convert the wind energy into electricity. The varying speeds at which the wind blows causes the rotors to rotate furiously and drive a power generator inside the nacelle of the turbines. The nacelle is the life source of the wind turbine that includes a control unit and other mechanical components to alter the axes of the rotors or the direction of the blades as well as monitor the functioning of the wind turbine.

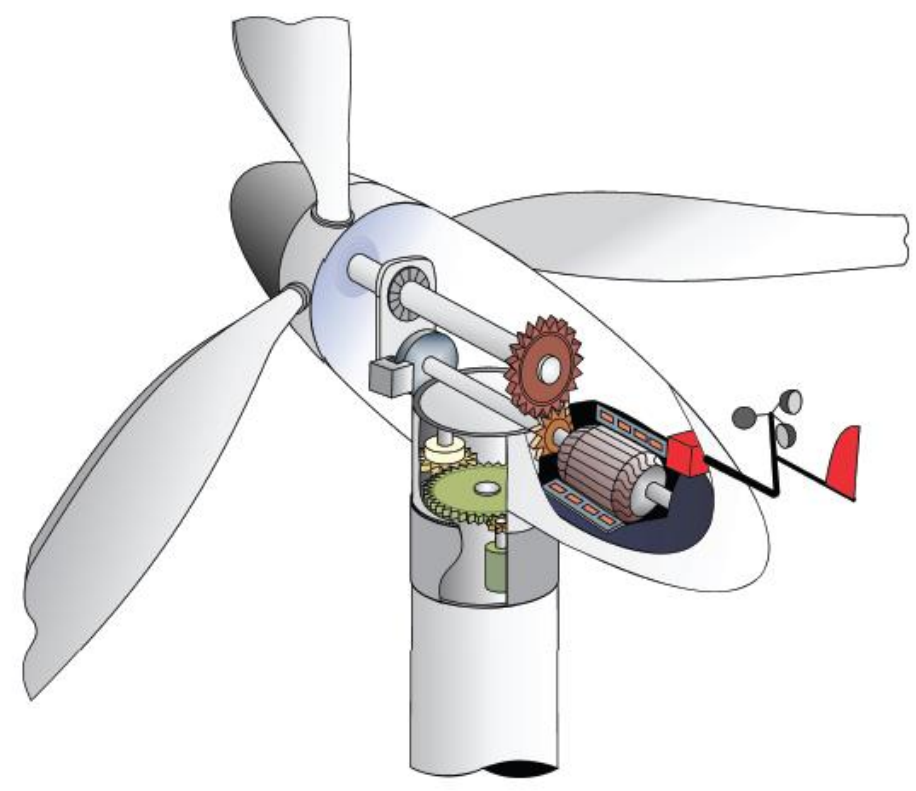

Figure F.14: Nacelle cross-section of the Wind Turbine system 
PLCs are typically used as these control units to provide the different conditions for monitoring and altering the wind turbine motors and rotor blades. In the following lab, you are required to simulate the nacelle of the wind turbine and calculate the power of the wind speed detected in the wind turbine. The specifications and constraints for the problem are as follows:

i) You are required to activate the nacelle to rotate the blades in the clockwise or anticlockwise direction. In the event that both the directions are activated, the nacelle must stay still and not rotate in either direction. The interlocking concept must be used here.

ii) The wind speed must be indicated using indicator lights in the case of a strong wind $($ Speed $>=60 \mathrm{kmph})$ and weak wind speed (Speed $<=15 \mathrm{kmph})$ and Normal at all other speeds.

iii) Finally, the program must enter into a sub-routine to calculate the Power produced by the Wind Turbine. In order to calculate this, the following data has been provided:

Blade Length, $1=52 \mathrm{~m}$

Wind Speed, $v=$ Variable (Obtained this from a timer module preset at 3000s)

Air Density, rho $=1.23 \mathrm{~kg} / \mathrm{m}^{3}$

Power Co-efficient, $\mathrm{Cp}=0.4$.

Power $=(1 / 2)^{*}\left(\rho \mathrm{Av}^{3} \mathrm{Cp}\right)$, where $\mathrm{A}=$ Area $\left(\pi \mathrm{r}^{2}\right)$

Table F.3: Address Map for Lab 14

\begin{tabular}{|c|l|l|}
\hline Address & \multicolumn{1}{|c|}{ Type } & \multicolumn{1}{c|}{ Description/Functional Requirement } \\
\hline I:0/1 & External Input & Toggle Switch NO - Start \\
\hline I:0/2 & External Input & Toggle Switch NO - Switch 1 \\
\hline I:0/3 & External Input & Toggle Switch NO - Switch 2 \\
\hline I:0/5 & External Input & Pushbutton NO - Reset \\
\hline O:0/1 & External Output & Indicator Light - Clockwise Status \\
\hline O:0/3 & External Output & Indicator Light - Counter-Clockwise Status \\
\hline O:0/5 & External Output & Indicator Light - Strong Wind Speed \\
\hline O:0/6 & External Output & Indicator Light - Normal Wind Speed \\
\hline O:0/7 & External Output & Indicator Light - Weak Wind Speed \\
\hline
\end{tabular}




\section{F3.2 Laboratory Fourteen - Solution}
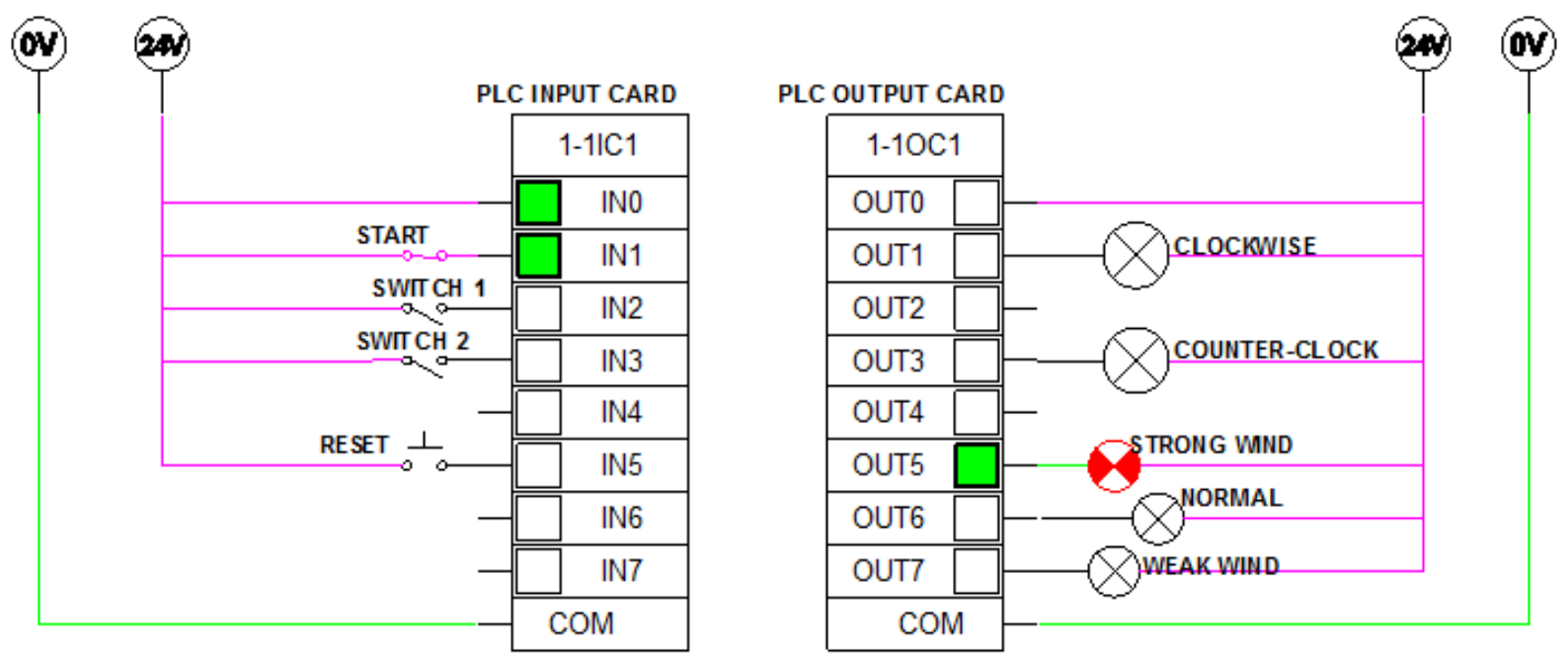

Figure F.15: PLC I/O Wiring Diagram for Lab 14 (currently in Strong Wind Status)

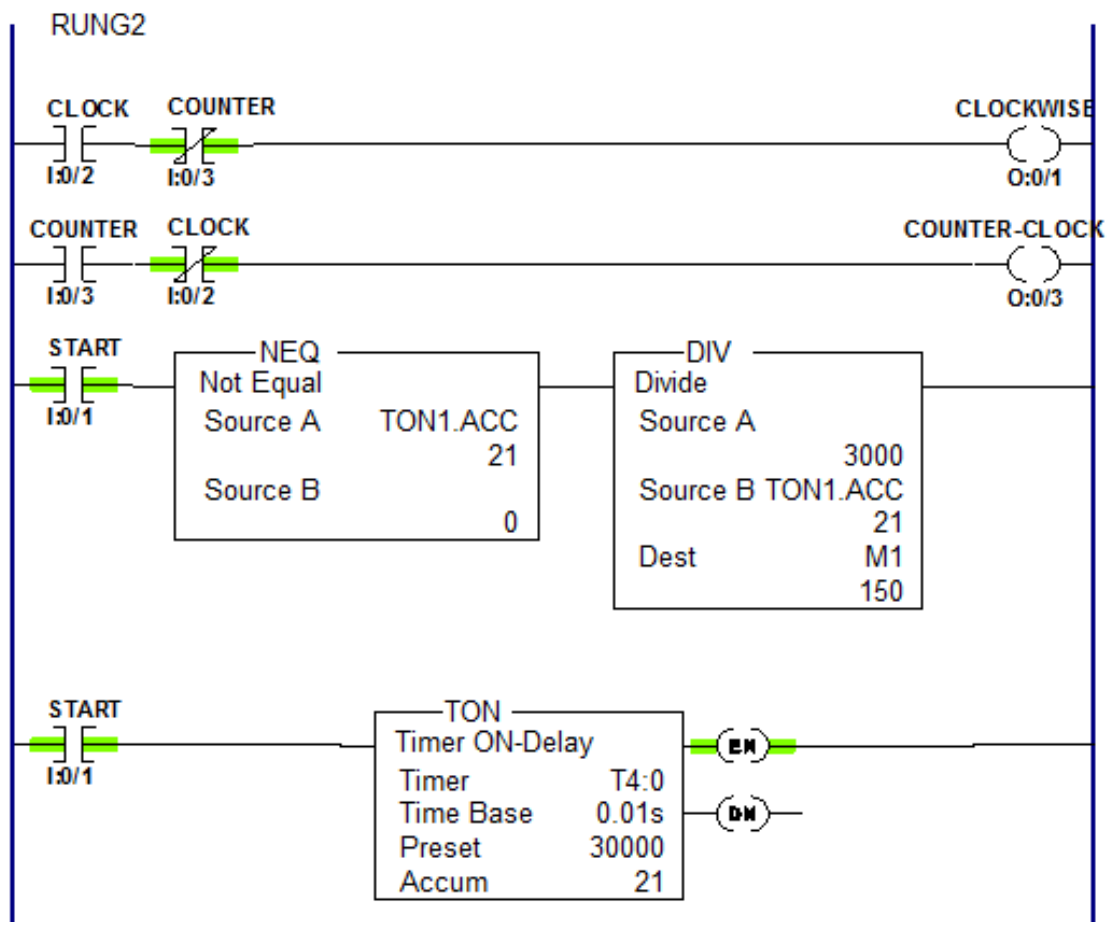

Figure F.16: Part 1 of the Ladder Logic Program for Lab 14 (currently in Strong Wind Status) 


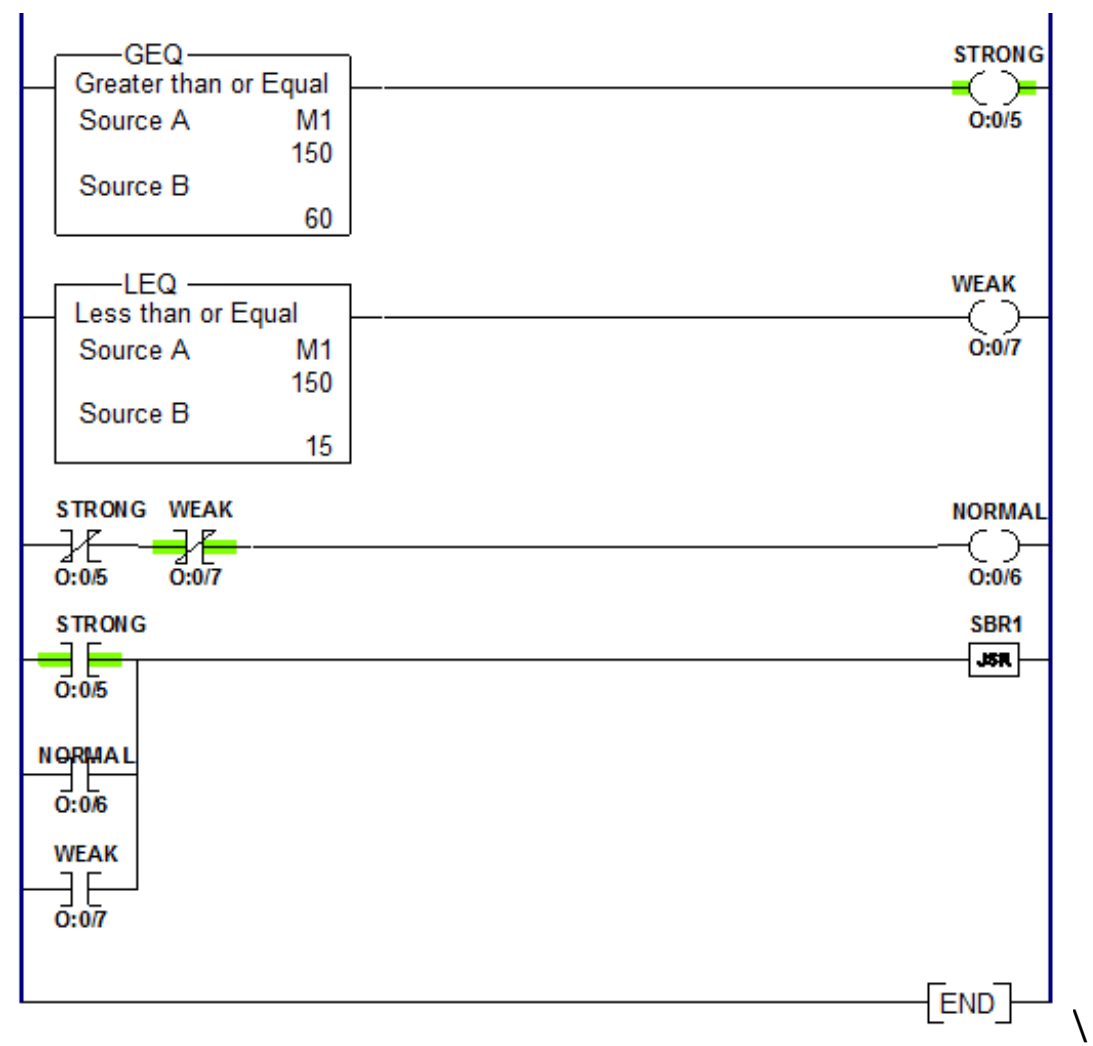

Figure F.17: Part 2 of the Ladder Logic Program for Lab 14 (currently in Strong Wind Status)

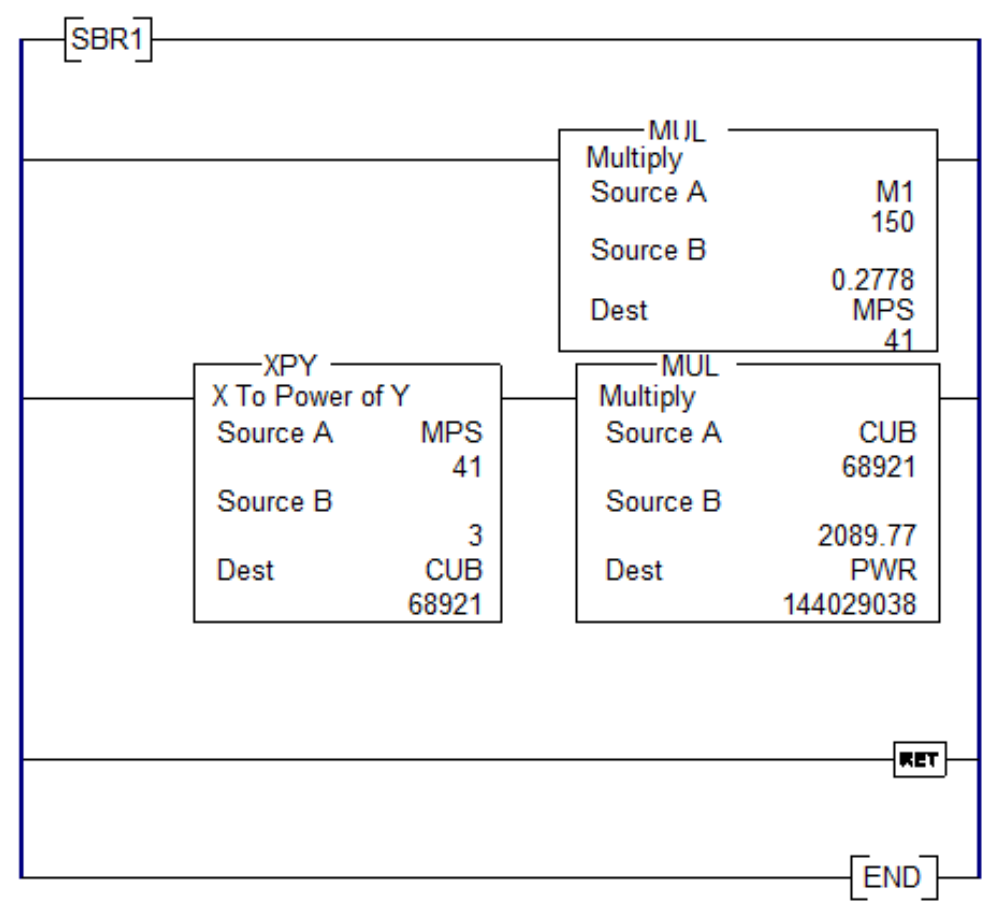

Figure F.18: Sub-Routine Ladder Logic Program for Lab 14 (displaying the Power Calculation) 


\section{BIBLIOGRAPHY}

[1] Bayindir, R., Sefa, I., Colak, I., \& Bektas, “A. Fault Detection and Protection of Induction Motors Using Sensors", IEEE Transactions on Energy Conversion, 23. (2008). http://ieeexplore.ieee.org.ezproxy.lib.ryerson.ca/stamp/stamp.jsp?tp=\&arnumber=4603060

[2] Wong, Kiing Ing, and Teck Ung Siaw. "PLC and SCADA Laboratory Experiments for a Final Year Instrumentation Course." International Journal of Information and Education Technology, vol. $5.11(2015)$

[3] Bassily, Hany, et al. "A Mechatronics Educational Laboratory - Programmable Logic Controllers and Material Handling Experiments." Mechatronics 17.9 (2007): 480-8.

[4] Domínguez, M., et al. "Remote Laboratory of a Quadruple Tank Process for Learning in Control Engineering using Different Industrial Controllers." Computer Applications in Engineering Education, vol. 22.3 (2014): 375-86.

[5] Petruzella, Frank D. Programmable Logic Controllers. 4th ed. New York: McGraw-Hill, 2005.

[6] Gabor, G., Zmranda, D., Gyorodi, C., \& Dale, S. Redundancy method used in PLC related applications. Soft Computing Applications, 2009. SOFA , 119-126.

[7] Bayindir, Ramazan, and Yucel Cetinceviz. "A Water Pumping Control System with a Programmable Logic Controller (PLC) and Industrial Wireless Modules for Industrial plants-An Experimental Setup." ISA transactions 50.2 (2011): 321-8.

[8] $\mathrm{Hu}, \mathrm{W}$. A knowledge-based real-time diagnostic system for PLC controlled manufacturing systems. Systems, Man, and Cybernetics, 1999. IEEE SMC'99 Conference Proceedings. IEEE International Conference, 4. 
[9] Alayón, S., C. González, and P. Toledo. "A Laboratory Experiment for Teaching Automation Inspired by the Smart Home." Computer Applications in Engineering Education 21.S1 (2013): E121-31.

[10] L. Moreno, C. S. Gonza'lez, I. Castilla, E. J. Gonza'lez, and J. F.Sigut, “Applying a constructivism and collaborative methodological approach in engineering education", Computer Education,.47 (2007), 891-915.

[11] "PLC - Ladder Logic." Automation Studio ${ }^{\mathrm{TM}}$ Educational Edition. Famic Technologies Inc. Web. <http://www.famictech.com/edu/index.html>

[12] "Introduction to Basic Ladder Logic Instructions." KronoTech. 2003. Web. $<$ http://www.kronotech.com/>.

[13] Fuada, Syifaul, Miftakhul Huda, and Rista Aprilowena. "A study Basic Programmable Logic Controller (PLC) for Effective Learning." International Journal of Computers \& Technology, vol. 3.3 (2012).

[14] "SMC Pneumatics Canada / Actuators | SMC Corporation of America." SMC Pneumatics Canada / Actuators, SMC Corporation of America. Web. 15 July 2015.

[15] The Virtual Lab @ NITK Surathkal. Center for System Design. Web. $<$ http://solve.nitk.ac.in/>.

[16] Wild, Peter, Brian Surgenor, and Gene Zak. "The Mechatronics Laboratory Experience." Mechatronics 12.2 (2002): 207-15.

[17] Moller T., et al. "PLC based control of remote laboratory experiments". 2008 11th International Biennial Baltic Electronics Conference. IEEE; 2008:273-276. 
[18] Yousif I Al Mashhadany. "Design and Implement of a Programmable Logic Controller (PLC) for Classical Control Laboratory." Intelligent Control and Automation 3.1 (2012): 44-9.

[19] Ferrater-Simon, C., et al. "A Remote Laboratory Platform for Electrical Drive Control using Programmable Logic Controllers." IEEE Transactions on Education 52.3 (2009): 425-35.

[20]Zhang, Hehua, et al. "New Strategies for Reliability Analysis of Programmable Logic Controllers." Mathematical and Computer Modelling 55.7-8 (2012; 2011): 1916-31.

[21] W.Bolton, "Programmable Logic Controllers",Newnes publications, Fifth Edition. (2009).

[22] Garry Dunning, "Introduction to Programmable Logic Controllers", 2nd Edition, Thomson, ISBN: 981-240-625-5.

[23] Jung, I., "PLC Control Logic Error Monitoring and Prediction Using Neural Network", Natural Computation, ICNC '08. Fourth International Conference, 2, 484 - 488, 2008

[24] Puchun, A., \& Jinhai, L. A PLC-Based Data Acquisition System with Adaptive Threshold Algorithm for Leak Detection of Oil Pipeline.Chinese Automation Congress (CAC),10.1109/CAC.2013.6775843, 801-805.

[25] Alok, V., \& Goel, A. "To study the different industrial applications of PLC through ladder diagrams. National Institute Of Technology Rourkela”. (2011)

[26] PLC Applications Wind Turbine. In Mechatronics (1st ed.). Quebec: Festo Didactic Ltée/Ltd, 2009, ISBN 978-2-89640-332-5

[27] Engineer On A Disk. (n.d.). Retrieved September 3, (2015). http://engineeronadisk.com/V2/book_integration/engineeronadisk-11.html

[28] Sheet Metal. (n.d.). Retrieved September 3, 2015. http://www.micro-machineshop.com/sheet_metal.htm 
[29] Shear Press. (n.d.). Retrieved September 3, 2015. http://atomictoasters.com/wpcontent/uploads/2011/10/Planing_machine_with_electric_motor_drive_Rankin_Kennedy_ Modern_Engines_Vol_VI.jpg

[30] Wind Turbine Power Calculations. (n.d.). RWE Npower Renewables. Retrieved from http://www.raeng.org.uk/publications/other/23-wind-turbine

[31] John Bird, "Engineering Mathematics", Fifth Edition, published by Elsevier Ltd. (2007). 


\section{GLOSSARY}

PLC Programmable Logic Controller

SCADA Supervisory Control and Data Acquisition

I/O Input/Output

GUI Graphical User Interface

HMI Human Machine Interface

PBL Problem Based Learning

OTL Output Latch

OTU Output Unlatch

OTE Output Energize

XIC Examine-If-Closed

XIO Examine-If-Open

TOF Timer-Off Delay

TON Timer-On Delay

PCB Printed Circuit Board 
